\title{
Revision of the bee genus Capicola (Hymenoptera: Apoidea: Melittidae) distributed in the Southwest of Africa
}

\author{
Denis MiCHEZ ${ }^{1}$, Connal EARDLEY², Michael KUHLMANN³ and Sébastien PATINY ${ }^{4}$ \\ ${ }^{1}$ Université de Mons-Hainaut, Laboratoire de Zoologie, Pentagone, Av. du Champs de Mars, 6, B-7000 Mons, Belgium; \\ e-mail: denis.michez@umh.ac.be \\ ${ }^{2}$ Plant Protection Research Institute, Private Bag X134, 0121 Queenswood, Pretoria, South Africa; e-mail: eardleyc@arc.agric.za \\ ${ }^{3}$ Institute of Landscape-Ecology, University of Münster, Robert-Koch-Str. 26, D-48149 Münster, Germany; \\ e-mail:kuhlmmi@uni-muenster.de
}

${ }^{4}$ Faculté universitaire des Sciences agronomiques de Gembloux, Unité d'Entomologie fonctionnelle et évolutive, Passage des Déportés 2, B-5030 Gembloux, Belgium; e-mail: patiny.s@fsagx.ac.be

Key words. Hymenoptera, Melittidae, Capicola, systematic, new species, new synonymies, distribution, host plants, key, Afrotropical region

\begin{abstract}
The bee family Melittidae (Hymenoptera: Apoidea) includes 14 genera. Most have been reviewed but not the small African genus Capicola. The present work aims to fill this gap. Based on the revision of the type material and 1272 additional specimens, we provide a comprehensive synonymic and annotated catalogue of the 12 Capicola species sensu Michener (1981). Lectotypes are designated for the previously established species Capicola braunsiana (= C. obscura syn. n.), C. cinctiventris, C. femorata, C. flavitarsis and C. rufiventris. Moreover, C. danforthi sp. n., C. flavicara sp. n., C. gessorum sp. n., C. micheneri sp. n., and C. richtersveldensis sp. n., are newly described, as well as the previously unknown female of $C$. nanula, male of $C$. nigerrima and male of C. rhodostoma. New synonymies are proposed for C. braunsiana $(=C$. obscura $)$ and C. flavitarsis $(=C$. cinctiventris $)$. An original dataset of 20 morphological characters is used for the inference of the 12 species' phylogenetic relationship. A key to species is provided. In addition, the ranges of the 12 taxa are mapped and discussed. This paper contributes to a global revision of the Melit tidae.
\end{abstract}

\section{INTRODUCTION}

The family Melittidae is subdivided into four subfamilies Dasypodainae, Macropidinae, Meganomiinae and Melittinae (Engel, 2001). They include 14 genera ranging worldwide, except in Australia and South America (Michener, 2000). Most of these genera have been reviewed (Stage, 1966; Michener, 1981; Whitehead \& Steiner, 2001; Michez et al., 2004a,b; Michez \& Patiny, 2005, 2006) but the revision of Capicola Friese, 1911 is still needed.

Capicola is restricted to southern Africa, but is closely related to the Neartic genus Hesperapis Cockerell, 1898 (Michener, 1981; Engel, 2005). Both, Hesperapis and Capicola share morphological features, notably the shape of stigma, the two sub-marginal cells (the first longer than the second), the galea comb inserted in front of the maxillary palpus insertion and the scopa restricted to the outer face of hind tibia and basitarsus. On the contrary, the two genera differ in the shape of the pygidial plate, which displays a strong longitudinal median, well-defined, usually elevated, area in Capicola females.

Friese (1911) described the genus Capicola based on $C$. braunsiana Friese, 1911, and placed it in Panurginae (due to the presence of two submarginal cells and a short glossa). Later, the same author (Friese, 1912, 1925), followed by Cockerell (1932a, b, 1934, 1936a), described 15 additional species in Capicola, Hesperapis and Rhinochaetula Friese, 1912. Michener (1981) synonymised most of these species acknowledging six species in the genus. This author also proposed the placement of Capicola within Dasypodaini and its subdivision into two subgenera: Capicola s.str. (including $C$. braunsiana, $C$. cinctiventris Friese, 1912, C. flavitarsis Friese, 1912, C. nanula Cockerell, 1936, C. rufiventris Friese, 1912) and Capicoloides Michener, 1981 (for C. aliciae Cockerell, 1932). More recently, Capicola and Capicoloides were placed as two subgenera among the seven already acknowledged in Hesperapis (Michener, 2000). At the supra-generic level, Engel (2005) proposed to place Hesperapis s. 1. (including Capicola and Capicoloides) in the subtribe Hesperapina, together with Eremaphanta Popov, 1940.

Knowledge of the life history of Capicola is still sketchy. Only $C$. braunsiana was studied by Rozen (1974). The nesting area studied by Rozen is described as sandy, treeless, with numerous widely spaced desert plants (principally Mesembryanthemum sp., Aizoaceae). The nest itself is very simple with a main ground tunnel giving rise to a series of linear cells. On the other hand, in a recent work, Gess \& Gess (2004) described floral choices for 12 species of Capicola, highlighting their specialized floral choices. According to this work, six species are oligolectic on Wahlenbergia sp. (Campanulaceae), five other on Mesembryanthemum sp. and a twelfth on Indigofera sp. (Fabaceae) and Limeum sp. (Molluginaceae). Unfortunately, the latter authors did not 
make reference to the taxonomic identity of the studied bee species, making impossible any further discussion.

In the present paper, we provide a comprehensive synonymic and annotated catalogue of Capicola sensu Michener (1981) based on the study of the type material and 1272 additional non-type specimens. Lectotypes are designated for the previously described species C. braunsiana, C. cinctiventris, C. femorata Friese, 1925, C. flavitarsis and C. rufiventris. Moreover, C. danforthi sp. n., $C$. flavicara sp. n., C. gessorum sp. n., C. micheneri sp. n. and $C$. richtersveldensis sp. n., are described, as well as the previously unknown female of $C$. nanula, male of $C$. nigerrima Cockerell, 1932 and male of C. rhodostoma Cockerell, 1932. The phylogenetic relationships among the 12 species are inferred based on a dataset of 20 morphological characters. A key to species is given. In addition, the geographic ranges of these taxa are mapped and discussed. The present paper contributes to a global revision of the Melittidae.

\section{MATERIAL AND METHODS}

\section{Studied material}

The type material of Capicola is located in following institutions: Albany Museum, Grahamstown, South Africa (AMGS); American Museum of Natural History New York, USA (AMNH); Cornell University, Ithaca, USA (CUIC); Natural History Museum, London, United Kingdom (NHMN); Plant Protection Research Institute, Pretoria, South Africa (SANC); Museum für Naturk. der Humboldt-Univ., Berlin, Germany (ZMHB); Iziko South African Museum, Cape Town, South Africa (SAMC).

All types were reviewed, except that of $C$. callura Cockerell 1932 (not located). Additional material was studied from collections of: Albany Museum, AMGS, 273 spec.; American Museum of Natural History, AMNH, 289 spec.; Cornell University, CUIC, 21 spec.; Iziko South African Museum, SAMC, 478 spec.; Museum für Naturk. der Humboldt-Univ., ZMHB, 3 spec.; National Museum Windhoek Namibia, NMWN, 74 spec.; Natural History Museum, NHMN, 51 spec.; Oberösterreichisches Landesmuseums, OOLL, 6 spec.; Plant Protection Research Institute, SANC, 49 spec.; Kuhlmann, University of Münster, UM, 21 spec.

\section{Taxonomic and systematic study}

Species concept

In the present paper we define taxa using a morphological species concept, too little information being available to consider eco-, etho- or pheno-species.

\section{Morphological terms, abbreviations and illustrations}

We used the glossary of Harris (1979) for description of the surface sculpture and Michener's (2000) for morphology. Puncture density is given as the relationship between puncture diameter (d) and the spaces between them (i), such as i $>d$.

The following abbreviations were used for morphological structures: antennal segment $-\mathrm{A}(\mathrm{A} 1=$ scape $)$; tibia $-\mathrm{Tb}$; femur - F; Basitarsus - Bt; Metasomal sternum - S; Metasomal tergum $-\mathrm{T}$; pygidial plate $-\mathrm{Pp}$; body length $-\mathrm{L}$; maximum width $-\mathrm{W}$. The integument ultrastructures were studied using SEM (JEOL JSM-6100) associated to the software Semafore (JEOL, Sollentuna, Sweden).

\section{Cladistic analysis}

Taxa. The 12 described species were included in the cladistic analysis (dataset in Table 1). Dasypoda hirtipes (Fabricius, 1793) and Hesperapis regularis (Cresson, 1878) (Dasypodainae, Dasypodaini) were used as outgroups.

Characters. 20 morphological characters were included in the analysis.

Female and male

1. Head: (0) as wide as long; (1) wider than long; (2) longer than wide.

2. Outer surface of galea: (0) sculptured; (1) smooth.

3. Labial palpus: (0) as long as maxillary palpus; (1) shorter than maxillary palpus.

4. Clypeus: (0) black; (1) reddish to yellow.

5. Clypeus: (0) width equal to length; (1) twice as wide as long; (2) two and a half times as wide as long; (3) three times as wide as long.

6 . Base of propodeal triangle: (0) rugose; (1) shiny.

7. Disc of T2-4: (0) flat, without basal concavity; (1) with basal concavity.

Female

8. Clypeus: (0) densely punctate $(i<d)$, at least basally; (1) sparsely punctate $(i>3 d)$.

9. Propodeum seen in profile: (0) with declivous basal area; (1) with horizontal basal area.

10. Spurs of Tb2: (0) without outstanding sharp spine; (1) with outstanding sharp spines.

11. Outer surface of Tb3: (0) without large, blunt spine; (1) with large, blunt spine.

12. Pp: (0) without median elevated area; (1) with median elevated area weakly raised, consisting of a pair of median subparallel ridges, area between these ridges concave; (2) with a strongly elevated median area.

Male

13. Clypeus: (0) without median groove; (1) with median groove.

14. A5: (0) longer than wide; (1) width subequal to greater than length.

15. Tb3: (0) not expanded apically; (1) expanded apically.

16. Disc of S6: (0) without medio-longitudinal groove; (1) with medio-longitudinal groove.

17. S6: (0) without medio-apical process; (1) with medio-apical curved blade; (2) with narrow medio-apical teeth that are half as long as S6 disc; (3) with large medio-apical teeth as long as S6.

18. S7: (0) disc not narrowed, apex generally emarginate; (1) main part narrowed medially.

19. S7: (0) apical lobes not diverging; (1) apical lobes diverging. 20. Gonostylus: (0) without groove; (1) with narrow hairy groove (with parallel margins); (2) with large hairy groove (margin of groove not parallel); (3) with large hairless groove.

Analysis. The dataset (Table 1) was analysed in two ways using PAUP $4.0 \mathrm{~b} 10$ (Swofford, 2001). A heuristic parsimony search was first made with Dasypoda hirtipes and Hesperapis regularis as outgroups (ingroup $=12$ Capicola species). The starting tree was obtained by stepwise addition. 10,000 random additional sequences were performed during the search. The branch swapping option was kept as default (TBR). All minimal trees found during branch swapping were kept. The most parsimonious trees yielded were used to compute a strict and a 50\% majority rule consensus. The effect of successive weighting method (along ten steps of reweighting) was tested using the same analytical framework. In this case, the most parsimonious 
TABLE 1. Character-state matrix for cladistic analysis.

\begin{tabular}{lccccccccccccccccccccc}
\hline & 01 & 02 & 03 & 04 & 05 & 06 & 07 & 08 & 09 & 10 & 11 & 12 & 13 & 14 & 15 & 16 & 17 & 18 & 19 & 20 \\
\hline$\quad$ Outgroup & & & & & & & & & & & & & & & & & & \\
Dasypoda hirtipes & 0 & 0 & 0 & 0 & 0 & 0 & 0 & 0 & 0 & 0 & 0 & 0 & 0 & 0 & 0 & 0 & 0 & 0 & 0 & 0 \\
$\begin{array}{l}\text { Hesperapis regularis } \\
\quad 1\end{array}$ & 0 & 0 & 0 & 0 & 0 & 0 & 0 & 1 & 0 & 0 & 0 & 0 & 0 & 0 & 0 & 0 & 0 & 0 & 0 \\
$\quad$ Ingroup & & & & & & & & & & & & & & & & & & & \\
Capicola aliciae & 1 & 0 & 0 & 0 & 1 & 0 & 0 & 1 & 1 & 1 & 1 & 2 & 1 & 1 & 0 & 0 & 3 & 1 & 1 & 0 \\
C. braunsiana & 0 & 0 & 0 & 0 & 1 & 0 & 0 & 1 & 1 & 1 & 0 & 1 & 0 & 1 & 0 & 1 & 2 & 0 & 0 & 1 \\
C. danforthi & 1 & 1 & 1 & 0 & 2 & 1 & 1 & 0 & 0 & 0 & 0 & 2 & 0 & 0 & 1 & 1 & 1 & 0 & 0 & 2 \\
C. flavicara & 2 & 0 & 0 & 1 & 1 & 0 & 0 & 0 & 0 & 1 & 0 & 0 & 0 & 1 & 0 & 1 & 2 & 0 & 0 & 1 \\
C. flavitarsis & 1 & 0 & 1 & 0 & 2 & 1 & 0 & 0 & 0 & 1 & 0 & 2 & 0 & 1 & 1 & 1 & 1 & 0 & 0 & 2 \\
C. gessorum & 1 & 1 & 1 & 0 & 2 & 1 & 1 & 0 & 0 & 0 & 0 & 1 & 1 & 1 & 1 & 1 & 1 & 0 & 0 & 3 \\
C. micheneri & 1 & 0 & 1 & 0 & 3 & 0 & 0 & 1 & 1 & 1 & 1 & 2 & 0 & 1 & 0 & 0 & 3 & 1 & 1 & 1 \\
C. nanula & 1 & 0 & 1 & 0 & 3 & 0 & 0 & 1 & 1 & 1 & 0 & 1 & 1 & 1 & 0 & 1 & 2 & 0 & 0 & 1 \\
C. nigerrima & 1 & 1 & 1 & 0 & 2 & 1 & 1 & 0 & 0 & 1 & 0 & 2 & 1 & 0 & 1 & 1 & 1 & 0 & 0 & 2 \\
C. rhodostoma & 0 & 0 & 0 & 1 & 1 & 1 & 0 & 0 & 0 & 1 & 0 & 1 & 0 & 1 & 0 & 1 & 2 & 0 & 0 & 1 \\
C. richtersveldensis & 1 & 0 & 1 & 0 & 2 & 1 & 1 & 0 & 0 & 1 & 0 & 2 & 0 & 0 & 1 & 1 & 1 & 0 & 0 & 2 \\
C. rufiventris & 1 & 0 & 1 & 0 & 1 & 0 & 0 & 0 & 0 & 1 & 0 & 2 & 0 & 1 & 1 & 1 & 1 & 0 & 0 & 3 \\
\hline
\end{tabular}

trees yielded on the final (tenth) step were used to draw a strict and a $50 \%$ majority rule consensus. The bootstrap (150 replicates) support values were computed both considering the characters of equal weight and after the last reweighting step.

Alternatively, an exhaustive search was also performed (ALLTREES). H. regularis (outgroup) and $C$. rufiventris (ingroup) were cancelled from the dataset, to bring the number of taxa to 12, which is the upper limit for exhaustive search in PAUP.

\section{Biogeography, phenology and host plant affiliation}

We synthesised the biogeography, phenology and host plant information in a catalogue. Species are listed following alphabetical order. The synonyms are quoted under the priority species name in chronological order with references to their original description and subsequent citations. In addition, we give the holotype depository and the locus typicus for each species.

The non-type specimens information is also quoted using the following standardisation of the labels' data: locality, coordinates (or conventional coordinates in decimal degrees when not on the label), date, collector, plants visited and depository.

The displayed maps synthesize the biogeographical information of the material examined as well as biogeographical data published with species description (Friese, 1912, 1925; Cockerell, 1932a, b, 1934, 1936a, b). These biogeographical data have been included in BDFGM (Banque de données fauniques Gembloux-Mons). They were processed using Data Fauna Flora 2.0 (Barbier et al., 2000). Conventional geographic coordinates for the records were obtained from the numeric gazetteer included in the software (CFFGazet). Data were mapped using Carto Fauna Flora 2.0 (Barbier \& Rasmont, 2000). A Gall geographical projection was used for mapping (Barbier \& Rasmont, 2000).

The labelling data provided information for the host plant of 370 specimens of Capicola. Most of these data are from the expeditions of F.W. and S.K. Gess (AMGS), and J.G. Rozen $(\mathrm{AMNH})$.

\section{RESULTS}

\section{Cladistic analysis}

The heuristic search based on the dataset (Table 1) yielded two equally parsimonious trees of length $=46, \mathrm{CI}$ $=0.6087$ and $\mathrm{RI}=0.7465$. The same search after ten steps of successive weighting of the characters yields one tree with the same topology, length $=21, \mathrm{CI}=0.784$ and $\mathrm{RI}=$ 0.8821 . The strict consensus of the trees from unweighted analysis is displayed in Fig. 100. The exhaustive search produces six trees, length $=44, \mathrm{CI}=0.6364$ and $\mathrm{RI}=$ 0.7288 (Fig. 101).

Based on the previous trees, three species-groups could be identified within Capicola (of which monophyly was strongly supported by the yielded topologies). The first of these groups include $C$. aliciae $+C$. michener $i=$ subgenus Capicoloides sensu Michener (1981)] associated with $C$. braunsiana, C. rhodostoma and $C$. nanula. The bootstrap value for $C$. aliciae $+C$. micheneri was notably high $(88,99$ with weighted characters). On the contrary, grouping within the three other species received only low support: 47 (77 after weighting) for basal node and 41 (57 after weighting) for C. nanula $+(C$. braunsiana + C. rhodostoma) (Fig. 100). This latter clade was not displayed by the consensus of the six trees yielded by an exhaustive search (Fig. 101). In this topology C. braunsiana, C. rhodostoma and $C$. nanula branched off a basal polytomy. The second group, C. rufiventris, C. flavitarsis, C. richtersveldensis, $C$. nigerrima, $C$. gessorum and $C$. danforthi, was better supported by bootstrap values. The basal node had a bootstrap support $=79$ (91 when characters were weighted) and all derived nodes received support higher than 50 (Fig. 100). The topology of this clade was conserved in the exhaustive search (Fig. 101). $C$. flavicara alone constituted the third group among Capicola.

The first species group is characterized by the derived state of the characters 8 and 9, namely the clypeus punc- 


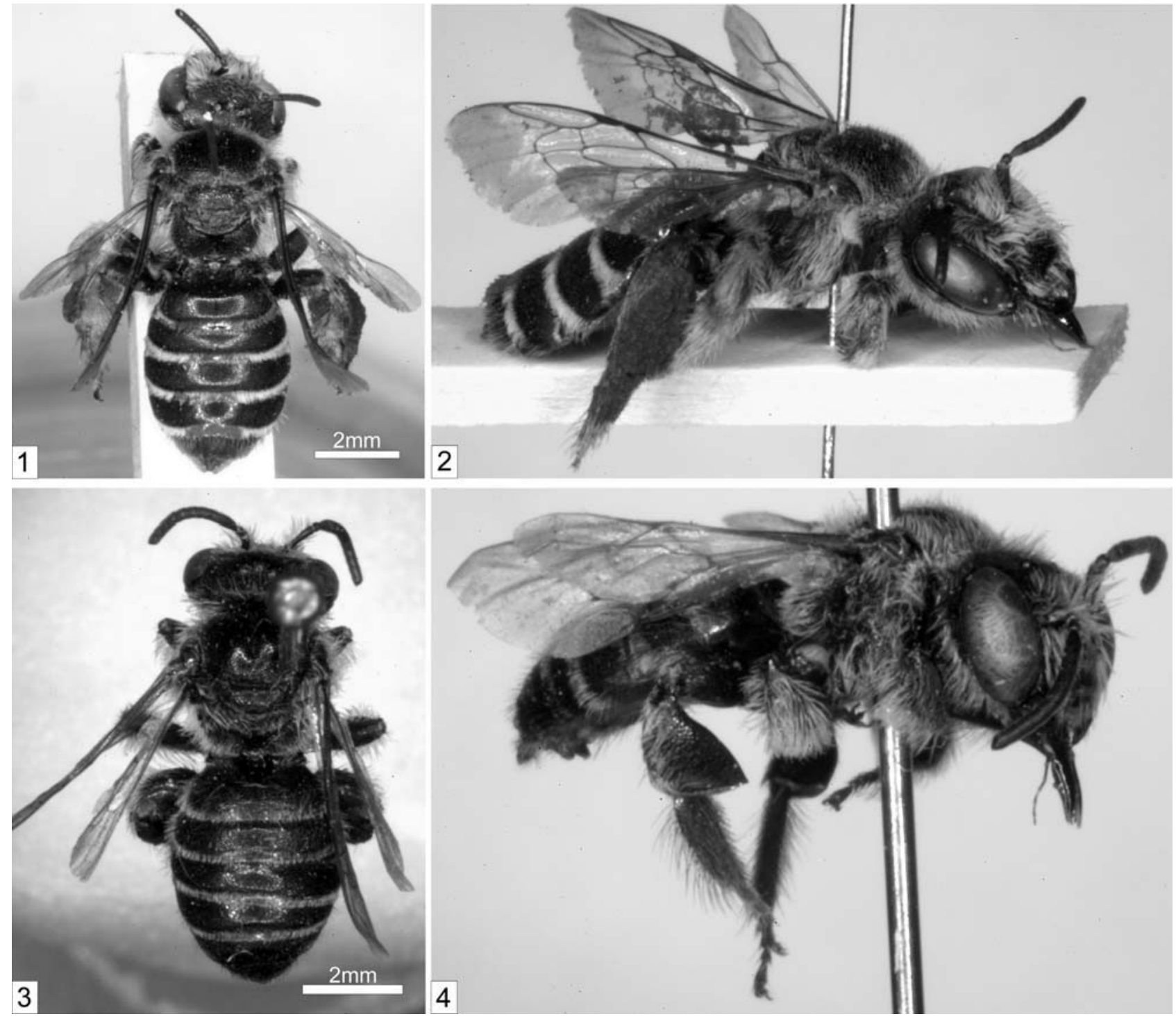

Figs 1-4. Capicola nigerrima. 1 - dorsal view of female habitus; 2 - lateral view of female habitus (scale as in Fig. 1); 3 - dorsal view of male habitus; 4 - lateral view of male habitus (scale as in Fig. 3).

tuation and the propodeum declivity. The second species group is characterized by the descriptors 15 , apical expansion of Tb3, 17, apical emargination of S6, and 20, presence of hairy grooves on gonostyli. C. flavicara is characterized by the yellow coloration of clypeus (character 4) and the shape of the head, which is longer than wide (character 1).

\section{Catalogue of species included in Capicola \\ Genus Capicola Friese, 1911}

Capicola Friese, 1911: 672. Type species: Capicola braunsiana Friese, 1911 (monobasic).

Capicola (Rhinochaetula) Friese, 1912: 185. Type species:

Capicola cinctiventris Friese, 1912 (= Capicola flavitarsis Friese, 1912).

Capicola (Capicola) Friese: Michener, 1981: 81-83.

Hesperapis (Capicola) Friese: Michener, 2000: 401-404.

Capicola (Capicoloides) Michener, 1981: 81-85. Type species: Capicola aliciae Cockerell, 1932 (monobasic).

Hesperapis (Capicoloides) Michener: Michener, 2000: 401-404.
Diagnosis. $q$ and $\widehat{\delta}$. Small to medium sized (4-12 $\mathrm{mm})$. Head generally wider than long. Galea comb in front of maxillary palpus insertion (Fig. 51). Propodeal triangle hairless, with horizontal (Fig. 44) or declivous basal area. Stigma less than twice as long as prestigma (Fig. 2). Two sub-marginal cells, first longer than second (Fig. 2). Second abscissa of Rs right angled (Fig. 2). Basitibial plate present (Figs 31, 43, 64, 80). Terga with apical fringes. + . Scopa restricted to outer surface of hind tibia and basitarsus (Figs 1-2). Keirotrichia extensively developed on inner surface of $\mathrm{Tb} 3$ and Bt3 (Fig. 45). Pp with longitudinal median, well defined, usually elevated area (Figs 9-10, 18, 30, 48-49, 58-59, 91). ठ. Pp absent. S6 commonly tetralobed (two medio-apical and two lateroapical processes), lobes hairy (Figs 11, 20-21, 26, 32, 38, $52,60,66-67,71,78,84,87)$. Gonostylus fused to gonocoxite, usually with a medio-longitudinal hairy groove (Figs 14-16, 23-25, 29, 35-37, 55-57, 63, 68-70, 74-76, 82-83) 


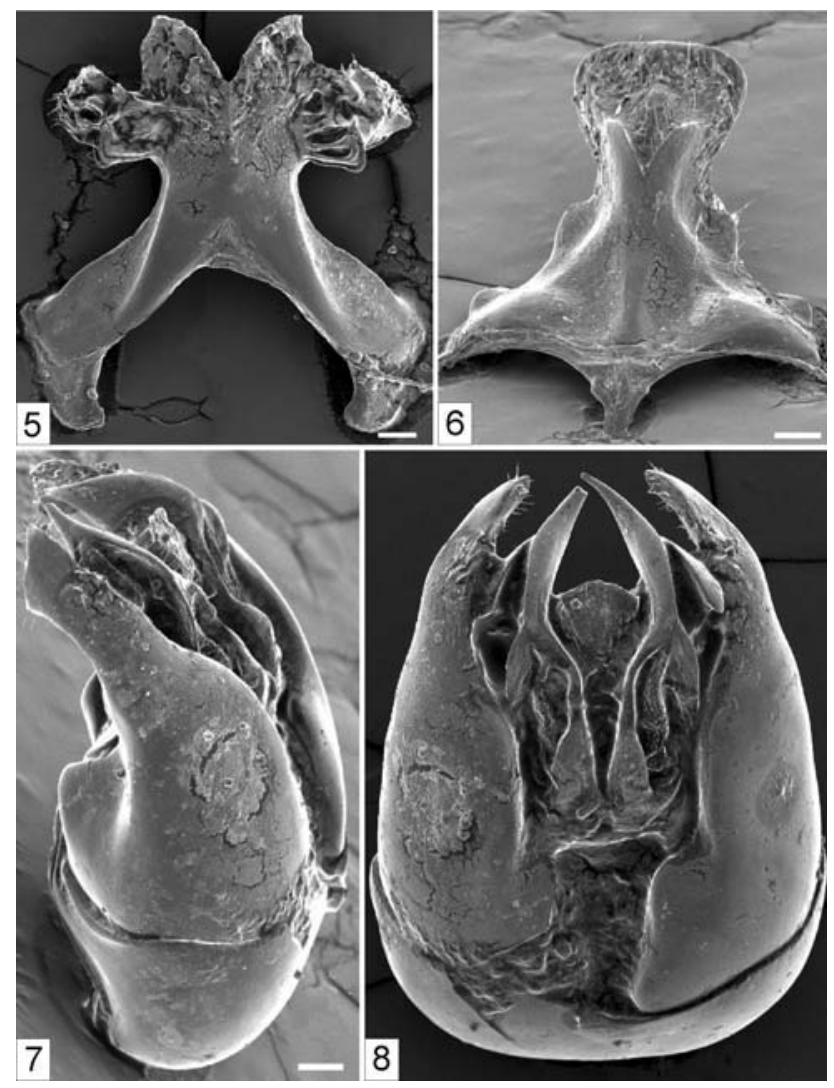

Figs 5-8. Capicola aliciae, đ. 5 - ventral view of sternum 7 $($ scale $=100 \mu \mathrm{m}) ; 6-$ ventral view of sternum $8($ scale $=100$ $\mu \mathrm{m}) ; 7-$ lateral view of genitalia $($ scale $=100 \mu \mathrm{m}) ; 8-$ dorsal view of genitalia (scale as in Fig. 7).

\section{Capicola aliciae Cockerell, 1932}

Capicola aliciae Cockerell, 1932b: 174-175, ㅇ․ Hesperapis aliciae (Cockerell): Cockerell, 1932c: 59.

Capicola (Capicoloides) aliciae Cockerell: Michener, 1981: 81, 83-85.

Hesperapis (Capicoloides) aliciae (Cockerell): Michener, 2000: 403.

Type material. Holotype + , South Africa, Calvinia $\left(31.47^{\circ} \mathrm{S}\right.$, $\left.19.78^{\circ} \mathrm{E}\right), \mathrm{NHMN}$. Original designation: "Calvinia, Cape Province".

Diagnosis (Figs 5-8). $q$ and $\delta$. Head wider than long. Labial palpus, maxillary palpus and glossa of similar lengths. Maxillary palpus shorter than galea. Outer surface of galea mat and sculptured. Clypeus twice as wide as long; with narrow median groove. Compound eyes converging slightly below. Posterior part of propodeal triangle glabrous. + . $\mathrm{L}=7.6 \mathrm{~mm}$. Clypeus glabrous with few weak punctures $(i>3 d)$. Mesoscutum and scutellum shiny, densely punctate $(\mathrm{i}=\mathrm{d})$. Basal part of propodeal triangle transversally carinate to granulose. Propodeum shiny. Legs 1-3 with yellowish hairs. Spurs of Tb2 with eight sharp outstanding spines. T1-T3 black and mat, with apical fringes. T1 with apical fringe sparser medially, sometimes interrupted. Prepygidial fimbria brown. Pp with median area strongly elevated. $\delta$ (Figs 5-8). L = $7.0 \mathrm{~mm}$. Body black. Clypeus covered with long, white, appressed hairs. Antennae ventrally reddish, dorsally brownish. S2-S4 with white, apical fringes. S3-S4 apically emarginated. S6 with wide medio-apical teeth. S8 with large median carina; column curved; apical plate oval shaped. S7-S8 and genitalia as illustrated in Figs 5-8.

Additional material examined (99 specimens). Namibia: 8 을 Namaskluft, $27.52^{\circ} \mathrm{S}, 16.52^{\circ} \mathrm{E}, 26 . \mathrm{ix} .2003$, F.W. \& S.K. Gess, AMGS; 19 , Rosh Pinah $\left(27.95^{\circ} \mathrm{S}, 16.77^{\circ} \mathrm{E}\right), 28 . x .1974$, R.H. Watmough, SANC; 1 \% , Klinghardtberge $\left(27.33^{\circ} \mathrm{S}\right.$, $\left.15.75^{\circ} \mathrm{E}\right), 19 . x .1974$, R.H. Watmough, SANC; 1 \% , Boom River Canyon, $4 \mathrm{~km}$ North Orange River, $28^{\circ} \mathrm{S}, 17.03^{\circ} \mathrm{E}, 200 \mathrm{~m}$, 25-30.x.1996, M\&A Wedd Expedition, SANC. South Africa: $3 \% / 1 \delta^{\circ}$, Richtersveld (Koeroegabvlakte), $28.11^{\circ} \mathrm{S}, 17.03^{\circ} \mathrm{E}$, 17.-24.ix.1995, on Prenia sladeniana, F.W. \& S.K. Gess, AMGS; 1 ऊ, Beaufort West $\left(32.35^{\circ} \mathrm{E}, 22.58^{\circ} \mathrm{E}\right), 28 . x i .1933$, Ogilvie, BMNH; 1ㅇ, idem, 29.xi.1933; 10, Graaf Reinet $\left(32.25^{\circ} \mathrm{S}, \quad 24.55^{\circ} \mathrm{E}\right), 27 . x .1931, \quad \mathrm{BMNH} ; 2$ 우, Nelspoort $\left(32.12^{\circ} \mathrm{S}, 23^{\circ} \mathrm{E}\right), 04 . x i i .1933$, Ogilvie, BMNH; 19 , Willowmore $\left(33.28^{\circ} \mathrm{S}, 23.48^{\circ} \mathrm{E}\right)$, Ogilvie, BMNH; 2 ㅇ․ $\left(31.38^{\circ} \mathrm{S}, 19.1^{\circ} \mathrm{E}\right), 06 . x i i .1996$, J.G. Rozen, AMNH; 7 \% , Garies $\left(30.55^{\circ} \mathrm{S}, 17.98^{\circ} \mathrm{E}\right), 21 . x .1969$, J.G. Rozen, AMNH; 19, Mesklip $\left(29.83^{\circ} \mathrm{S}, 17.85^{\circ} \mathrm{E}\right), 21 . x .1968$, J.G. Rozen, AMNH; 1 ㅇ, Leliefontein $\left(33.24^{\circ} \mathrm{S}, 19.17^{\circ} \mathrm{E}\right), 09 . x .2002$, Mayer, UM;

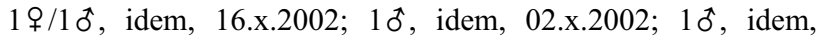
14.x.2002; $1 \% / 1 \delta^{\star}$, Koeroegab $\left(28.23^{\circ} \mathrm{S}, 17.03^{\circ} \mathrm{E}\right), 13 . x .2001$, on Prenia sladeniana, Mayer, UM; $1 \delta^{\circ}$, Remhoogte $\left(30.24^{\circ} \mathrm{S}\right.$, $\left.18.17^{\circ} \mathrm{E}\right), 16 . x .2001$, Mayer, UM; $1 \delta^{\Uparrow}$, idem, 25.x.2001; 2 이의, Richtersveld $\left(28.3^{\circ} \mathrm{S}, 17.08^{\circ} \mathrm{E}\right), 9 \mathrm{~km}$ ESE Kuboes, 25.ix.1995, F.W. \& S.K. Gess, AMGS; $1 \delta^{\widehat{ }}$, Richtersveld, northern Park between hills, NW Koeroegabvlakte $\left(28.25^{\circ} \mathrm{S}, 17.08^{\circ} \mathrm{E}\right)$, 14.ix.1995, F.W. \& S.K. Gess, AMGS; 2 \%, Richtersveld, Paradysberg $\left(28.31^{\circ} \mathrm{S}, 17.05^{\circ} \mathrm{E}\right), 11 . x .1974$, R.H. Watmough, SANC; $30^{\circ}$, Witwater, $30.23^{\circ} \mathrm{S}, 18.13^{\circ} \mathrm{E}, 4 .-6 . x .2003$, C. Meyer, AMGS; 40, Remhoogte, $30.24^{\circ} \mathrm{S}, \quad 18.17^{\circ} \mathrm{E}$,

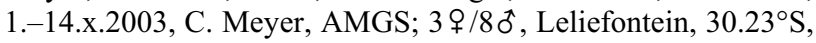
$18.17^{\circ} \mathrm{E}, 1 .-15 . \times .2003$, C. Meyer, AMGS; $19 / 1 \delta^{\hat{0}}$, idem, SANC; 4 q , Kenhardt area $\left(29.235^{\circ} \mathrm{S}, 21.15^{\circ} \mathrm{E}\right)$, x.1939, Museum Staff, SAMC; $11 \% / 9 ð$, Hester Malan Nature Reserve, Namaqualand, $29.66^{\circ} \mathrm{S} 17.98^{\circ} \mathrm{E}, 17 . \mathrm{x} .-12 . x i .1987$, M. Struck, SAMC; 1 \% , Moordenaars Karoo, Lammerfontein $\left(32.83^{\circ} \mathrm{S}\right.$, $\left.20.91^{\circ} \mathrm{E}\right)$, x.1952, Museum Expedition, SAMC; 19 , Tankwa Karoo, Kleinbrak, $\left(31.83^{\circ} \mathrm{S}, 25.4^{\circ} \mathrm{E}\right)$, xi.1952, Museum Expedition, SAMC; 19 , Tankwa Karoo, Renoster Rivier, $\left(31.33^{\circ} \mathrm{S}\right.$, $\left.20.3^{\circ} \mathrm{E}\right)$, xi.1952, Museum Expedition, SAMC; $19 / 2{ }^{\star}$, Lekkersing, Richtersveld, $29^{\circ} \mathrm{S}, 17.06^{\circ} \mathrm{E}, 12 . x .1966-29 . x i .1977$, V.B. Whitehead, SAMC; $10^{\hat{}}$, Witwater, $30.23^{\circ} \mathrm{S}, 18.13^{\circ} \mathrm{E}, 4 . x .2003$, C. Meyer, SAMC; $20^{\star}, 30 \mathrm{~km}$ South of Kamieskroon, $30.26^{\circ} \mathrm{S}$ $17.57^{\circ} \mathrm{E}, 28 . x i .1977$, V.B. Whitehead, SAMC.

Distribution (Fig. 95). South Western Namibia and widespread in the western and southern parts of South Africa.

Host plant (6 specimens). Aizoaceae, Prenia sladeniana $\left(4 \% / 2 \sigma^{*}\right)$.

First - last observations. 4.x.-06.xii.

\section{Capicola braunsiana Friese, 1911}

Capicola braunsiana Friese, 1911: 672-673, 이.

Capicola (Capicola) braunsiana Friese: Michener, 1981: 81-84; Michener, 2000: 403.

Hesperapis (Capicola) braunsiana (Friese): Eardley \& Urban, 2006: 162 .

Capicola aurescens Cockerell, 1932a: 457-458, ô.

Hesperapis aurescens (Cockerell): Cockerell, 1936a: 29.

Hesperapis (Capicola) aurescens (Cockerell): Michener, 1981: 83.

Hesperapis turneri Cockerell, 1934: 446-447, đ/우. 
Capicola (Capicola) turneri (Cockerell): Michener, 1981: 83. Hesperapis obscura Cockerell, 1934: 447-448, ․ Syn. n.

Type material of $\boldsymbol{C}$. braunsiana (designated here). Lectotype $\hat{\delta}$, paralectotype $q$, South Africa, Willowmore $\left(33.28^{\circ} \mathrm{S}\right.$, $23.48^{\circ} \mathrm{E}$ ), ZMHB. Original designation: "Willowmore, Kapland". Lectotype: ô (ZMBH); labels: $1^{\text {st }}$ printed on white paper "Willowmore Capland Dr. Brauns."; $2^{\text {nd }}$ handwritten with black ink on white paper "Capicola braunsiana 1910 Friese det."; $3^{\text {rd }}$ printed on red paper "Typus"; $4^{\text {th }}$ printed on white paper "Coll. Friese"; $5^{\text {th }}$ printed on white paper "Zool. Mus. Berlin"; $6^{\text {th }}$ printed on red paper "Capicola braunsiana Friese 1911 lectotype design Michez 2006". Paralectotype: \& $(\mathrm{ZMBH})$; labels: $1^{\text {st }}$ printed on white paper "Willowmore Capland Dr. Brauns."; $2^{\text {nd }}$ handwritten with black ink on white paper "Capicola braunsiana 1910 Friese det."; $3^{\text {rd }}$ printed on red paper "Typus"; $4^{\text {th }}$ printed on white paper "Coll. Friese"; $5^{\text {th }}$ printed on white paper "Zool. Mus. Berlin"; $6^{\text {th }}$ printed on red paper "Capicola braunsiana Friese 1911 lectotype design Michez 2006".

Type material of $\boldsymbol{C}$. aurescens. Holotype $\widehat{\delta}$, South Africa, Calvinia $\left(31.47^{\circ} \mathrm{S}, 19.78^{\circ} \mathrm{E}\right)$, NHMN. Original designation: "Calvinia, Cape Province".

Type material of $\boldsymbol{C}$. turneri. Holotype $\hat{0}$, cotype $\uparrow$, South Africa, Little Karoo (?), NHMN. Original designation: "Little Karoo, 38 miles east of Ceres, Cape Province".

Type material of $\boldsymbol{C}$. obscura. Holotype $q$, South Africa, Matjesfontein (?), NHMN. Original designation: "Matjesfontein, Cape Province".

Diagnosis (Figs 9-16). $q$ and $\delta$. Integument mostly black, ventral surface of antennal flagellum reddish-black, tarsi $2-3$ and $\mathrm{Tb} 3$ orangish. Head (including eyes) as wide as long. Labial palpus longer than glossa, as long as maxillary palpus and galea. Outer surface of galea mat and sculptured. Clypeus nearly hairless, about twice as wide as long, without median groove, sparsely punctate $(\mathrm{i}>3 \mathrm{~d})$. Mesoscutum densely and finely punctate $(\mathrm{i}<\mathrm{d})$. Propodeal triangle finely sculptured, shiny apically, with horizontal basal area. + (Figs 9-10). L $=8.4 \mathrm{~mm}$. Compound eyes parallel. Mesoscutum and scutellum with short appressed hairs. Spurs of Tb2 with 4-6 large, outstanding spines. T1-T3 black and mat. T1-T4 with continuous, white apical fringe. Prepygidial fimbria white to very pale brown. Pp with a pair of median subparallel carinae, area between carinae and lateral to them concave (Figs 9-10). of (Figs 11-16). L = $7.2 \mathrm{~mm}$. Eyes not enlarged. Antennae ventrally reddish, dorsally brownish. Scutellum mat, densely punctate $(\mathrm{i}<$ d). T1-T5 with white apical fringe. Hind leg without segments enlarged or toothed. S6 with short, narrow medioapical spiny processes (Fig. 11). Apex of S7 weakly concave, with small lobes (Fig. 12). S8 with apical plate subtriangular. S8 and genitalia as illustrated in Figs 13-16.

Additional material examined (278 specimens). Namibia: 3 $\$ / 1 \mathrm{o}^{\widehat{ }}$, Keetmanshoop $\left(26.58^{\circ} \mathrm{S}, 18.13^{\circ} \mathrm{E}\right), 01 . x i .1968$, J.G.

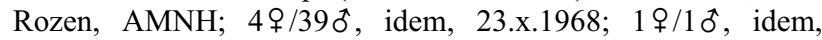
22.x.1968; 3 이어, idem, 30.x.1968; 19,14 km S Rosh Pinah, near Orange River, $28.17^{\circ} \mathrm{S}, 16.55^{\circ} \mathrm{E}, 27 . x .1974$, R. Watmough, SANC; 29 , between Rosh Pinah $\left(28.17^{\circ} \mathrm{S}, 16.55^{\circ} \mathrm{E}\right)$ and Orange River, 28.x.1974, R. Watmough, SANC; 1 \%, Luderitz, Sinclair Mines $\left(25.7^{\circ} \mathrm{S}, 16.37^{\circ} \mathrm{E}\right), 7 . x .1972$, NMWN; 2 으, Churutabis (Bethanie) $\left(27.43^{\circ} \mathrm{S}, 17.47^{\circ} \mathrm{E}\right), 4 .-12 . x .1974, \mathrm{NMWN}$; 8웅 Gaiaus (Karasberg) $\left(27.33^{\circ} \mathrm{S}, 18.75^{\circ} \mathrm{E}\right), 13 .-17 . x .1974$,
NMWN; 2 , Barby (Bethanie) $\left(25.85^{\circ} \mathrm{S}, 16.55^{\circ} \mathrm{E}\right), \mathrm{NMWN}$. South Africa: $3 \% / 40^{\top}$, Beaufort West $\left(32.35^{\circ} \mathrm{S}, 22.58^{\circ} \mathrm{E}\right)$, 29.xi.1933, Ogilvie, BMNH; 2ㅇ, idem, 25.xi.1933; 3 , idem, 28.XI.1931; $1 \widehat{\delta}^{\widehat{ }}$, Willowmore $\left(33.28^{\circ} \mathrm{S}, 23.48^{\circ} \mathrm{E}\right)$, Brauns, $\mathrm{BMNH} ; 3 \delta^{\circ}$, Prince Albert Road $\left(32.98^{\circ} \mathrm{S}, 21.68^{\circ} \mathrm{E}\right)$, 26.xi.1931, Turner, BMNH; $2 \% / 20^{\star}$, Vanrhynsdorp $\left(31.61^{\circ} \mathrm{S}\right.$, $\left.18.73^{\circ} \mathrm{E}\right), 20 . x .1968$, J.G. Rozen, AMNH; $1 \% / 2{ }^{\star}$, idem, 23.x.1974; $10^{\circ}$, Kommetje $\left(34.13^{\circ} \mathrm{S}, 18.32^{\circ} \mathrm{E}\right)$, 06.xi.1968, J.G. Rozen, AMNH; $3 \% / 10^{\dagger}$, Steinkopf $\left(29.27^{\circ} \mathrm{S}, 17.73^{\circ} \mathrm{E}\right)$, 03.xi.1968, J.G. Rozen, AMNH; 1 \%, Porth Nolloth $\left(29.25^{\circ} \mathrm{S}\right.$, 16.87E), 30.x.1974, J.G. Rozen, AMNH; 10, idem, 26.x.1974;

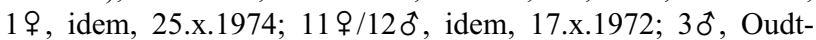
shoorn $\left(33.37 \mathrm{~S}, 22.13^{\circ} \mathrm{E}\right), 10 . x i 1.1996$, J.G. Rozen, AMNH; 6 $\% / 70^{\star}$, Steytlerville $\left(33.33^{\circ} \mathrm{S}, 24.35^{\circ} \mathrm{E}\right), 12 . x i .1968$, J.G. Rozen, AMNH; $30^{\circ}$, Prince Albert $\left(33.22^{\circ} \mathrm{S}, 22.03^{\circ} \mathrm{E}\right)$, 19.x.1972, J.G. Rozen, AMNH; 19 , Remhoogte $\left(30.4^{\circ} \mathrm{S}\right.$, $\left.18.28^{\circ} \mathrm{E}\right), 28 . x .2001$, Mayer, UM; $10^{\star}$, Soebatsfontein $\left(30.12^{\circ} \mathrm{S}\right.$, $\left.17.58^{\circ} \mathrm{E}\right)$, 08.-16.i.2002, Schmiedel, UM; $10^{\star}$, Knersvlakte $\left(31.17^{\circ} \mathrm{S}, 18.36^{\circ} \mathrm{E}\right), 14 . x i 1.2001$, Schmiedel, UM; 10 \% , Boom River Canyon, $4 \mathrm{~km}$ North Orange River, $28^{\circ} \mathrm{S}, 17.03^{\circ} \mathrm{E}, 200 \mathrm{~m}$, 25.-30.x.1996, M. \& A. Wedd Expedition, SANC; 1 ㅇ, Richtersveld, Foot of Hells Kloof $\left(28.3^{\circ} \mathrm{S}, 16.97^{\circ} \mathrm{E}\right), 10 . x .1974, \mathrm{R}$. Watmough, SANC; 7 \% , Vioolsdrif (Namaqualand) $\left(28.77^{\circ} \mathrm{S}\right.$, $17.65^{\circ}$ E), 9.x.1988, F.W. \& S.K. Gess, AMGS; 3 ㅇ, Oranjemund, $34 \mathrm{~km}$ from checkpoint, Sendelingsdrif road, $28.24^{\circ} \mathrm{S}$, $16.44^{\circ}$ E, 25.ix.1997, F.W. \& S.K. Gess, AMGS; 1 \% , between Annis and Dabie River, $28.20^{\circ} \mathrm{S}, 16.55^{\circ} \mathrm{E}, 20 . i x .1997$, F.W. \& S.K. Gess, AMGS; 4 $\$ / 3 \delta$, Hester Malan Nature Reserve, 29.66 ${ }^{\circ}$ S, $17.98^{\circ}$ E, 16.-26.x.1985, M. Struck, SAMC; 12 잉, idem, 1.x.-17.xi.1986, M. Struck, SAMC; 1 , idem, 31.x.1987, M. Struck, SAMC; $10^{\hat{*}}$, Williston, $31.35^{\circ} \mathrm{S}, 20.91^{\circ} \mathrm{E}$, 12.x.1966-30.xi.1978, V.B. Whitehead, SAMC; 1 t, Lekkersing, Richtersveld, $29^{\circ} \mathrm{S}, 17.06^{\circ} \mathrm{E}, 12 . x .1966-29 . x i .1977$, V.B. Whitehead, SAMC; 4 \% , Trawal, $31.88^{\circ} \mathrm{S}, 18.63^{\circ} \mathrm{E}$, 9.xi.1977, V.B. Whitehead, Mesembryanthemum crystalinum, SAMC; $1 \% / 10^{\circ}$, Spektakel, $29.65^{\circ} \mathrm{S}, 17.6^{\circ} \mathrm{E}, 30 \times x i .1977$, V.B. Whitehead, SAMC; $11^{\circ} / 1 \delta^{\circ}$, Witputs $\left(27.26^{\circ} \mathrm{S}, 20.48^{\circ} \mathrm{E}\right), 15 . x .1975$, V.B. Whitehead, on Mesembryanthemum sp., SAMC; 20 , Rooinek Pass $\left(33.20^{\circ} \mathrm{S}, 20.55^{\circ} \mathrm{E}\right)$, x.1952, Museum Expedition, SAMC; 1 \%, Albert District, between Burghersdorp and Nooitgedacht $\left(30.95^{\circ} \mathrm{S}, 26.25^{\circ} \mathrm{E}\right)$, x.1935, Museum Staff, SAMC; 2 \% $/ 2$ o $^{\star}$, Moordenaars Karoo, Lammerfontein, $32.96^{\circ} \mathrm{S}, 20.81^{\circ} \mathrm{E}$, x.1952, Museum Expedition, SAMC; 19 , between Nuwerus and Vanrhynsdorp $\left(31.22^{\circ} \mathrm{S}, 18.39^{\circ} \mathrm{E}\right)$, xi.1931, Museum Staff, SAMC; 49 , Graaff-Reinet, Brooklyn farm, $32.25^{\circ} \mathrm{S}, 24.4^{\circ} \mathrm{E}$, 3.i.1980, V.B. Whitehead, SAMC; $10^{\hat{}}$, Kirkwood $\left(33.41^{\circ} \mathrm{S}\right.$, $\left.26.44^{\circ} \mathrm{E}\right)$, 4.i.1980, V.B. Whitehead, on Mesembryanthemum grandiflorum, SAMC; $19,30 \mathrm{~km}$ E. of Nieuwoudtville $\left(31.38^{\circ} \mathrm{S}, 19.1^{\circ} \mathrm{E}\right), 26 . x i .1981$, V.B. Whitehead, on Mesembryanthemum sp., SAMC; 13 , Pofadder, Kenhardt Division, $29.13^{\circ} \mathrm{S}, 19.4^{\circ} \mathrm{E}, \mathrm{x} .1939$, Museum Staff, SAMC; 3 \% , Annisfontein, Richtersveld, $28.41^{\circ} \mathrm{S}, 16.88^{\circ} \mathrm{E}, 20 .-23 . x i .1975$, V.B. Whitehead, SAMC; 2 \% , Groenrivier Mountains, $64 \mathrm{~km}$ South of Hondeklipbaai, $30.51^{\circ} \mathrm{S}, 17.35^{\circ} \mathrm{E}, 30 \times x i .1976$, V.B. Whitehead, on Mesembryanthemum sp., SAMC; 39 , Vanrhynsdorp, $31.58^{\circ} \mathrm{S}, 18.73^{\circ} \mathrm{E}, 2 . x i .1976$, V.B. Whitehead, SAMC; 1 잉ㅇㅇ, Ladismith, $33.48^{\circ} \mathrm{S}, 21.26^{\circ} \mathrm{E}$, 10.xii.1978, V.B. Whitehead, on Mesembryanthemum sp., SAMC; $2 \hat{0}, 36 \mathrm{~km}$ North of Concordia, $29.23^{\circ} \mathrm{S}, 18^{\circ} \mathrm{E}, 20 . x i .1980$, V.B. Whitehead, SAMC; $1 \% / 1 \delta^{\widehat{\alpha}}, 48 \mathrm{~km}$ South of Vioolsdrift, $29.11^{\circ} \mathrm{S}, 17.8^{\circ} \mathrm{E}$, 11.x.1980, V.B. Whitehead, SAMC; $10^{\star}$, Obib, $28.08^{\circ} \mathrm{S}$, $16.75^{\circ}$ E, 28.x.1977, V.B. Whitehead, SAMC; 4 ㅇ, Calitzdorp, $33.53^{\circ} \mathrm{S}, 21.55^{\circ} \mathrm{E}, 28 . x i i .1978$, V.B. Whitehead, SAMC; 19 , idem, 29.xii.1978, V.B. Whitehead, SAMC; $5 q$, Cookhouse, $32.75^{\circ} \mathrm{S}, 25.81^{\circ} \mathrm{E}, 22 . x i .1983$, V.B. Whitehead, on Mesembryanthemum grandiflorum, SAMC; $2 \% / 4 \widehat{\imath}, 15 \mathrm{~km} \mathrm{~N}$. of Cook- 

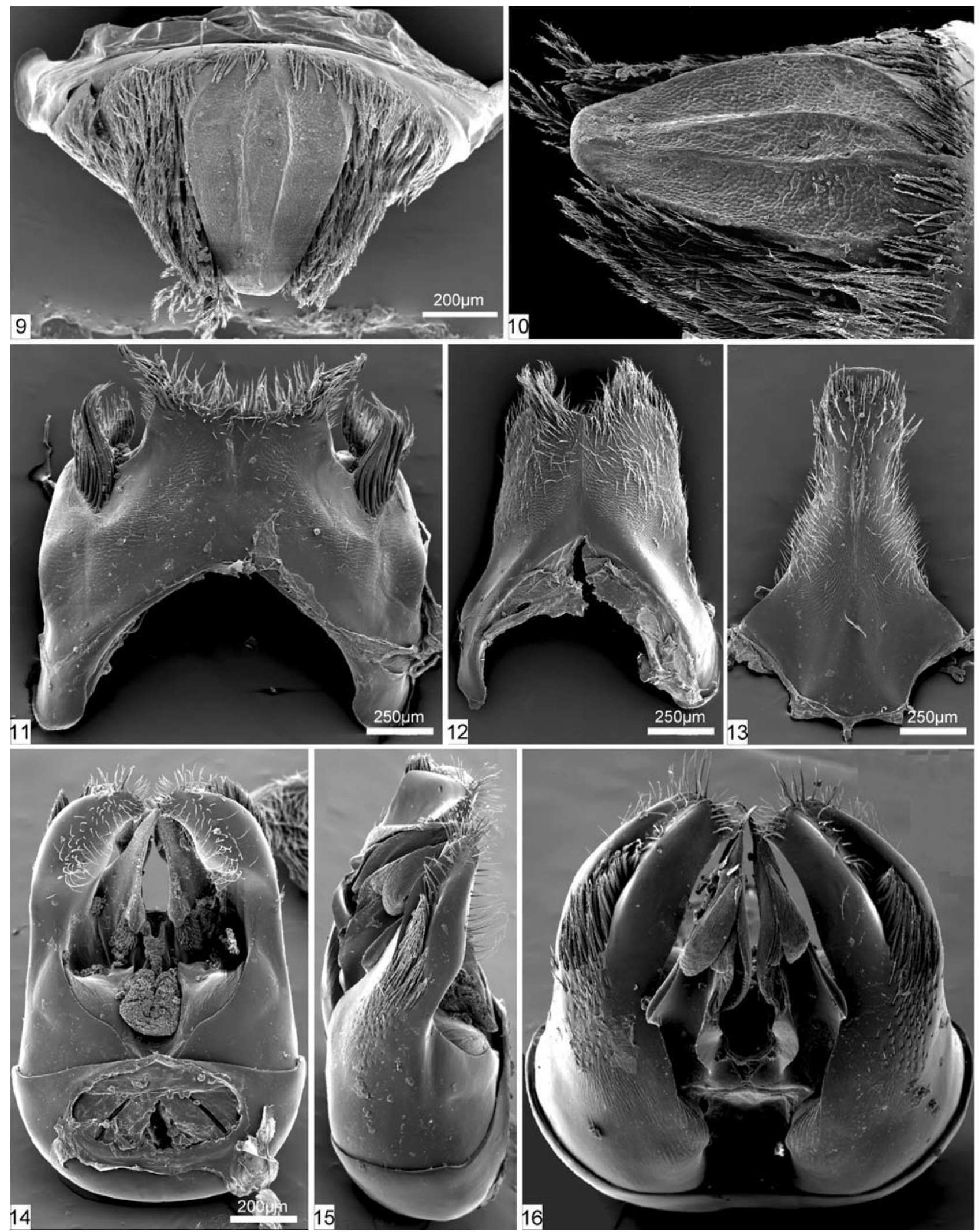

Figs 9-16. Capicola braunsiana. 9 - dorsal view of pygidial plate $\$ ; 10$ - lateral view of pygidial plate (scale as in Fig. 9); 11 ventral view of sternum $6 \hat{\delta} ; 12$ - ventral view of sternum $7 \bar{\delta} ; 13$ - ventral view of sternum $8 ; 14$ - ventral view of genitalia $\delta ; 15-$ lateral view of genitalia (scale as in Fig. 14) ôे; 16 - dorsal view of genitalia ồ (scale as in Fig. 16).

house $\left(32.75^{\circ} \mathrm{S}, 25.81^{\circ} \mathrm{E}\right), 2.1 .1985$, V.B. Whitehead, on Mesembryanthemum grandiflorum, SAMC; 4 으, Bracefield, $33.18^{\circ} \mathrm{S}$, 25.7 ${ }^{\circ}$ E, 23.xi.1983, V.B. Whitehead, on Mesembryanthemum grandiflorum, SAMC; $20^{\star}$, Kommadagga, $33.12^{\circ} \mathrm{S}, 25.91^{\circ} \mathrm{E}$, 26.xi.1982, V.B. Whitehead, SAMC; 2 \%, Richtersveld, $6 \mathrm{~km}$ South of Holgatrivier $\left(28.71^{\circ} \mathrm{S}, 17.03^{\circ} \mathrm{E}\right), 27 . x i .1986, \mathrm{M}$. 
Struck, SAMC; $2 \sigma^{\star}, 1.3 \mathrm{~km}$ East Khoebus Lekkersing $\left(29^{\circ} \mathrm{S}\right.$ 17. $\left.1^{\circ} \mathrm{E}\right), 27 . x i .1986$, M. Struck, SAMC; 5 \% $/ 30^{\star}$, Numees, Richtersveld, $28.28^{\circ} \mathrm{S}, 16.96^{\circ} \mathrm{E}$, 8.ix.1986, M. Struck, SAMC.

Distribution (Fig. 96). Widespread through southern Namibia and the western and southern regions of South Africa.

Host plant. (32 specimens). Aizoaceae, Mesembryanthemum sp. (5/7ठ), Mesembryanthemum crystalinum (4), Mesembryanthemum grandiflorum $(11+/ 5 \hat{0})$.

First - last observations. 20.ix.-30.i.

\section{Capicola danforthi Eardley sp. n.}

Diagnosis (Figs 17-25). 웅 and $\delta$. Head wider than long (Fig. 17). Labial palpus half as long as maxillary palpus, reaching beyond apex of glossa. Outer surface of galea smooth. Maxillary palpus as long as galea. Compound eyes more or less parallel. Clypeus two and a half times as wide as long. Clypeus, mesoscutum and scutellum shiny and deeply punctate. Propodeal triangle with declivous basal area. Disc of terga with basal concavity. o (Figs 17-18). L = $10.7 \mathrm{~mm}$. Propodeal triangle mostly shiny, weakly sculptured. Propodeum shiny. Legs 1-3 with yellow or orange hairs. Spurs of Tb2 without large spine. Scopa sparsely pubescent. Apical hair band on T1 continuous. T1-5 black, with shallow punctures. Prepygidial fimbria orange. Pp with a strong elevated median area (Fig. 18). 0 (Figs 19-25). L = $10.3 \mathrm{~mm}$. Integument mostly black. A4-13 black. A5 longer than wide. All legs black. Tb3 broadened apically, with a tooth between apical spurs (Fig. 19). S6 with medio-apical processes curved and erected ventrally (Figs 20-21). S7 disc very large (Fig. 22). Apical plate of S8 flattened. Genitalia as illustrated in Figs 23-25.

Description. + . Head. $\mathrm{L}=2.7 \mathrm{~mm} ; \mathrm{W}=3.4 \mathrm{~mm}$. Head wider than long (Fig. 17). Integument mostly black. Mandibles base, labrum and clypeus reddish-black. Clypeus, face, genal area and preoccipital ridge with white, erect hairs. Face shiny. Labial palpus half as long as maxillary palpus, reaching beyond apex of glossa. Maxillary palpus nearly as long as galea. Outer surface of galea smooth. Malar area shorter than A2. Face and vertex densely punctate. Clypeus two and a half times as wide as long; densely punctate $(i=d)$; without median groove. Compound eyes not converging ventrally. Antennae black to reddish-black. Mesosoma. $\mathrm{L}=2.1 \mathrm{~mm} . \mathrm{W}=2.7 \mathrm{~mm}$. Integument black. Mesoscutum and scutellum shiny, densely and distinctly punctate $(i=d)$. Mesepisternum and metanotum with long, erect white hairs. Propodeal triangle hairless, rugose anteriorly, glabrous posteriorly. Propodeum shiny, between dense punctures. Legs. Integument black, except tarsi 1-3 and Tb3 reddish. Vestiture orange. Spurs of $\mathrm{Tb} 2$ finely serrate, without large spines. Wings. Surface transparent. Two submarginal cells, first longer than second. Basal vein slightly curved. Metasoma. $\mathrm{L}=6.5 \mathrm{~mm}$. $\mathrm{W}=4.4 \mathrm{~mm}$. Terga and sterna black. Discs of terga with superficial punctures, with basal concavity. T1-T4 with white continuous apical fringes. Prepygidial fimbria orange. Sterna shiny. Sterna and terga with straight premarginal line. Pp with strong median raised area tapering to apex (Fig. 18). ठ․ Head. L $=2.8 \mathrm{~mm} . \mathrm{W}=3.6 \mathrm{~mm}$. Integument black. Mandible reddish-black. Head entirely covered in white, erect hairs.
Labrum swollen. Malar area shorter than A2. Clypeus trapezoidal, densely covered with white hairs, densely punctate $(\mathrm{i}<\mathrm{d})$. Eyes with inner margins converging ventrally. Face and vertex deeply punctate. Antennae black. A3 as long as A4. A5 longer than wide. Mesosoma. $\mathrm{L}=$ $3.9 \mathrm{~mm} . \mathrm{W}=2.6 \mathrm{~mm}$. Integument black, with long, white or orangish, erect hairs; median part of mesoscutum and scutellum a little less hairy. Nota sparsely punctulate. Propodeal triangle mat. Legs. Integument reddish-black. Outer surface with long, white or orange, erect hairs. Inner side of tarsi 1-3 with appressed, golden hairs. Tb3 enlarged (Fig. 19). Metasoma. $\mathrm{L}=5.4 \mathrm{~mm}$. W $=3.9 \mathrm{~mm}$. Integument black. T1-T4 covered with erect, white or orange hairs. S2-S5 hairless medially. Disc of terga regularly punctate $(i<d)$, with basal concavity. Apical margin of S5 straight. S6 with medio-apical processes curved ventrally (Figs 20-21). S7 body very large (Fig. 22). Apical plate of S8 flattened. Genitalia as illustrated in Figs 23-25.

Type material (211 specimens). Holotype $\widehat{\delta}, 152$ paratypes \&, 58 paratypes $\delta$; South Africa: $\delta$ holotype, $3 \$ / 10$, Arkoep Farm, $6 \mathrm{~km}$ N. Kamieskroon, $30.19^{\circ} \mathrm{S}, 17.56^{\circ} \mathrm{E}, 1 .-2 . x .1990$, C.D. Eardley, SANC; 2 \% , Richtersveld National Park, $28.18^{\circ} \mathrm{S}$, $16.58^{\circ} \mathrm{E}, 12 .-14.1 x .2001$, C. Eardley, SANC; $10^{\star}, 11 \mathrm{~km} \mathrm{~W}$

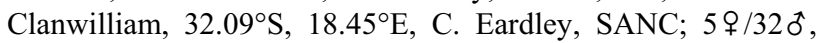
idem, 2.-8.x.1990, F.W. \& S.K. Gess, on Wahlenbergia annu-

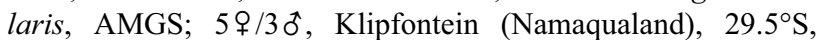
$17.47^{\circ}$ E, 14.x.1989, F.W. \& S.K. Gess, on Wahlenbergia cf. annularis, AMGS; 1 \% , Citrusdal $\left(32.6^{\circ} \mathrm{S}, 19.02^{\circ} \mathrm{E}\right)$, 16.x.1990, F.W. \& S.K. Gess, on Wahlenbergia annularis, AMGS; $7 \% / 10{ }^{\top}, \quad$ Voëlklip (Namaqualand), $29.45^{\circ} \mathrm{S}, \quad 17.22^{\circ} \mathrm{E}$, 2.-7.x.1994, F.W. \& S.K. Gess, on Wahlenbergia cf. annularis, AMGS; 2 $\$ / 30^{\circ}, 23 \mathrm{~km}$ from Citrusdal $\left(32.6^{\circ} \mathrm{S}, 19.02^{\circ} \mathrm{E}\right)$, on old road, 12.-13.x.1990, F.W. \& S.K. Gess, on Wahlenbergia annu-

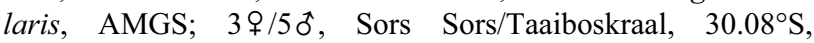
$18.01^{\circ} \mathrm{E}, 3 . \mathrm{x} .1995$, F.W. \& S.K. Gess, on Wahlenbergia annularis, AMGS; 3 ô, Kamieskroon (Namaqualand), Bakleikraal, $30.13^{\circ} \mathrm{S}, 18.03^{\circ} \mathrm{E}, 9 .-11 . x .1994$, F.W. \& S.K. Gess, on Wahlenbergia annularis, AMGS; 69 , between Kamieskroon and Springbok, $29.88^{\circ} \mathrm{S}, 17.52^{\circ} \mathrm{E}$, x.1939, Museum Staff, SAMC; 115 ㅇ, Rooinek Pass $\left(33.20^{\circ} \mathrm{S}, 20.55^{\circ} \mathrm{E}\right)$, x.1952, Museum Expedition, SAMC; 3 ㅇ, Klip Vlei, Garies, $\left(33.26^{\circ} \mathrm{S}, 18.35^{\circ} \mathrm{E}\right)$, xi.1931, Museum Staff, SAMC.

Etymology. Named after Prof. B.N. Danforth who collected the type material of $C$. richtersveldensis and chose that name for the species.

Distribution (Fig. 97). Western region of South Africa.

Host plant (79 specimens). Campanulaceae, Wahlenbergia annularis $(10 \% / 43 \hat{\delta})$ and Wahlenbergia cf. annularis $(13$ \% 13 ô) $)$.

First - last observations. 14.ix to an unspecified date in November.

\section{Capicola flavicara Eardley sp. $n$.}

Diagnosis (Figs 26-29). $q$ and $\delta$. Head longer than wide. Integument of antenna, supraclypeal area, clypeus, labrum, mandible, scutum, scutellum, fore and middle legs yellow or orange. Maxillary palpus a little shorter than labial palpus, the latter not quite reaching end of glossa. Maxillary palpus a little shorter than galea, which is subequal in length to labial palpus. Outer surface of galea sculptured. Compound eyes with upper and lower 

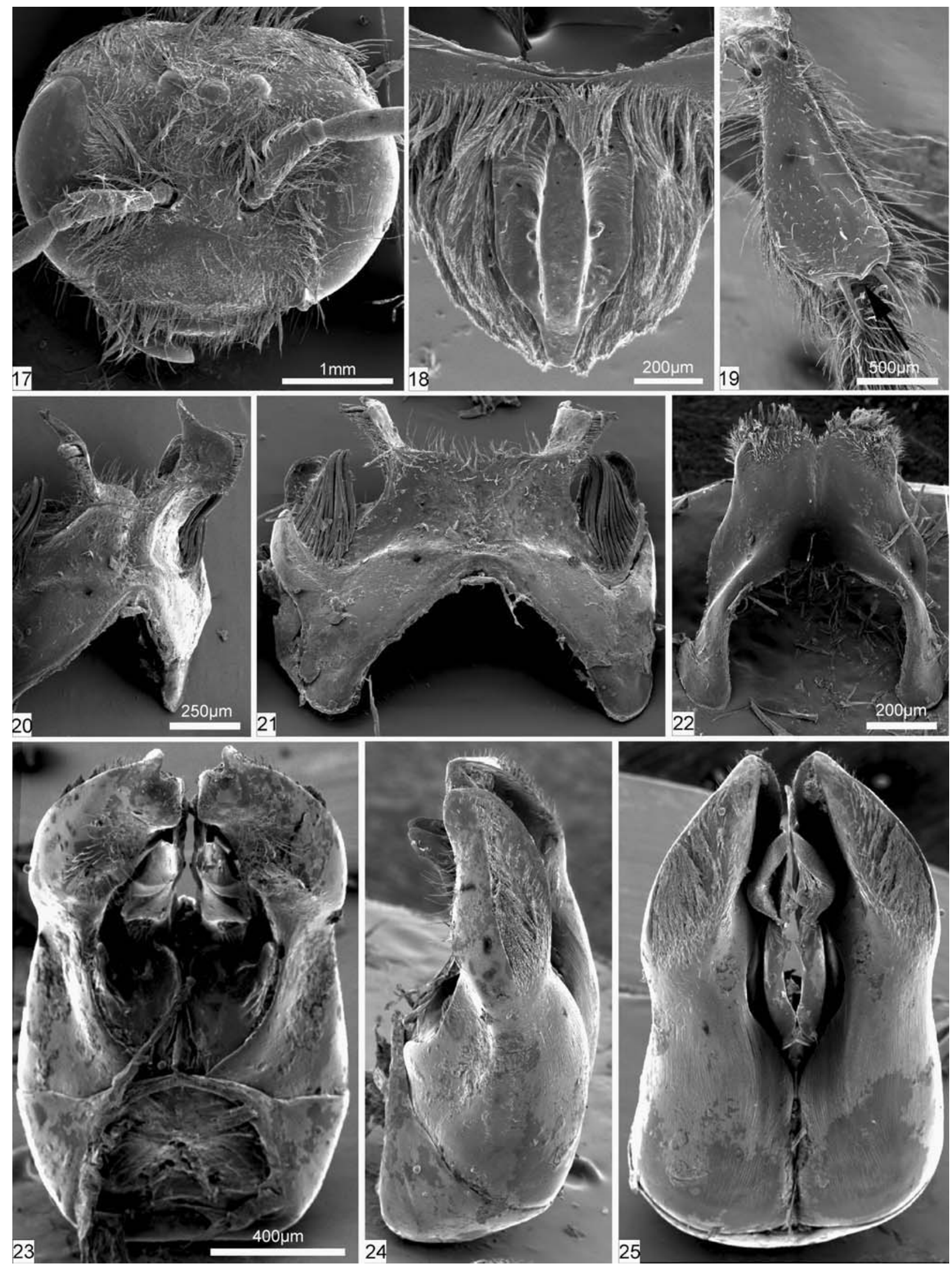

Fig. 17-25. Capicola danforthi sp. n. 17 - frontal view of head $\$ ; 18$ - dorsal view of pygidial plate $\$ ; 19$ - inner view of hind tibia $\hat{\delta}$ with tooth between apical spurs (arrow); 20 - lateral view of sternum $7 \hat{\delta} ; 21$ - ventral view of sternum $7 \hat{\delta}$ (scale as in Fig. 20); 22 - ventral view of sternum 6; 23 - ventral view of genitalia $\widehat{\delta} ; 24$ - lateral view of genitalia $\delta$ (scale as in Fig. 23); 25 dorsal view of genitalia $\hat{0}$ (scale as in Fig. 23). 

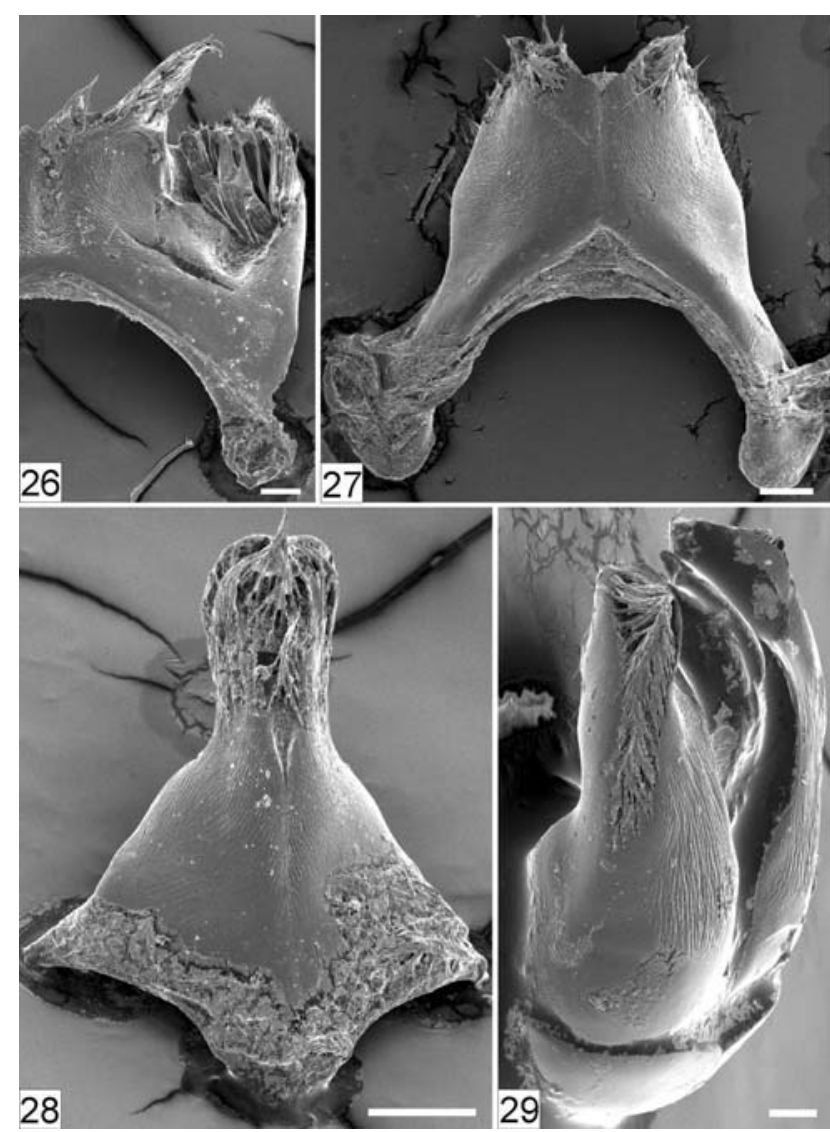

Figs 26-29. Capicola flavicara sp. n., đ. 26 - ventral view of sternum $6($ scale $=100 \mu \mathrm{m}) ; 27-$ ventral view of sternum 7 $($ scale $=100 \mu \mathrm{m}) ; 28-$ ventral view of sternum $8($ scale $=200$ $\mu \mathrm{m}) ; 29$ - lateral view of genitalia $($ scale $=100 \mu \mathrm{m})$.

interorbital distances subequal. Clypeus about twice as wide as long. Clypeus, mesoscutum and scutellum shiny, with very small, shallow punctures. $+\mathrm{L}=6.8 \mathrm{~mm}$. T1-T2 and S1-S2 yellow or orange, remainder dull black. Propodeal triangle mostly shiny, with reticulate sculpture. Remainder of propodeum mat. Legs 1-3 with whitish vestiture, except orange under hind basitarsus. Spurs of $\mathrm{Tb} 2$ with eight very small spines. Scopa densely pubescent proximally, sparsely pubescent distally. Apical fringes on T1-4 continuous. Prepygidial fimbria black to brownish. Pp gently concave, without medio-longitudinal carinae. ô (Figs 26-29). L = 5.4 mm. Propodeum, T1-T4 and S1-S4 yellow or orange, remainder dull black. Eyes not prominent. A5 as long as wide. $\mathrm{Tb} 3$ not broadened. S6 with short medio-apical teeth (Fig. 26). S7-8 and genitalia as illustrated in Figs 27-29.

Description. ?. Head. $\mathrm{L}=2.2 \mathrm{~mm} ; \mathrm{W}=2.0 \mathrm{~mm}$. Head longer than wide. Integument of antenna, supraclypeal area, clypeus, labrum and mandible yellow or orange, remainder black. Face, genal area and preoccipital ridge with white hairs. Face shiny, with fine punctation. Maxillary palpus a little shorter than labial palpus, the latter not quite reaching end of glossa. Maxillary palpus a little shorter than galea, which is subequal in length to labial palpus. Outer surface of galea scluptured and mat. Malar area shorter than A2. Face and vertex densely but weakly punctate $(\mathrm{i}=2 \mathrm{~d})$. Clypeus about twice as wide as long; with many small, shallow punctures, without mediolongitudinal groove. Compound eyes not converging. Mesosoma. $\mathrm{L}=2.4 \mathrm{~mm} . \mathrm{W}=1.6 \mathrm{~mm}$. Integument of pronotum, scutum and scutellum orange, remainder black. Mesoscutum and scutellum shiny, densely but weakly punctate $(\mathrm{i}=2 \mathrm{~d})$. Mesepisterna and metanotum with long, white hairs. Propodeum seen in profile with horizontal basal area. Propodeal triangle hairless, mostly shiny, with basal reticulate sculpture; remainder of propodeum mat. Legs. Integument of legs 1-2 mostly orange, leg 3 mostly blackish; vestiture white. Inner surface of tarsus 3 with orange hairs. Spurs of $\mathrm{Tb} 2$ with about eight very small spines. Wings. Surface transparent. Two submarginal cells, the first longer than the second. Basal vein slightly curved. Metasoma. $\mathrm{L}=3.6 \mathrm{~mm}$. $\mathrm{W}=2.2 \mathrm{~mm}$. $\mathrm{T} 1-\mathrm{T} 2$ and S1-S2 reddish, remainder of metasoma dull black, with reticulate sculpture and weak punctures. T1-T4 with white, continuous apical fringes. Prepygidial fimbria black. Premarginal lines curved anteriorly medially. Pp shallowly concave, reddish, without medio-longitudinal carinae. ${ }^{\dagger}$. Head. $\mathrm{L}=1.9 \mathrm{~mm} . \mathrm{W}=1.8 \mathrm{~mm}$. Head slightly longer than wide. Integument of antenna, supraclypeal area, clypeus, labrum and mandible, yellow or orange, remainder dull black. Face, genal area and preoccipital ridge with white hairs. Vertex shiny, with fine punctation. Maxillary palpus a little shorter than labial palpus, the latter not quite reaching end of glossa. Maxillary palpus a little shorter than galea, which is subequal in length to labial palpus. Galea sculptured and mat. Labrum not swollen. Malar area shorter than A2. Clypeus about twice as wide as long, with small, shallow punctures, without a medio-longitudinal groove. Compound eyes converge slightly ventrally. Face and vertex with small, shallow punctures. A3 longer than A4. Mesosoma. L = $2.0 \mathrm{~mm} . \mathrm{W}=1.3 \mathrm{~mm}$. Integument of scutum, scutellum, propodeum orange; remainder dull black. Vestiture generally long and white (partly damaged and cannot be fully described). Mesoscutum, scutellum, metanotum and propodeum with small, shallow and dense punctures $(i<d)$. Anterior part of propodeal triangle with reticulate sculpture. Legs. Legs 1-2 orange, leg 3 dull black. Sculpture reticulate. Vestiture white, except ventral surface of tarsus 3 blackish-orange. Tb3 not modified. Wings. Surface brown. Two submarginal cells; first longer than second. Basal vein slightly curved. Metasoma. $\mathrm{L}=3.1 \mathrm{~mm} . \mathrm{W}=$ $1.8 \mathrm{~mm}$. Integument T1-T4 and S1-S4 yellowish-orange, remainder reddish-black. T1 with white, medially interrupted, apical fringe; T2-T4 with white, uninterrupted, apical fringes. Reticulate sculpture and sparse, fine punctures. S6 with short medio-apical teeth (Fig. 26). S7-8 and genitalia as illustrated (Figs 27-29).

Type material (4 specimens). Holotype $\delta, 2$ paratypes $\$, 1$ paratype $\hat{\delta}$; South Africa: $19 / 1 \hat{\delta}$, Annisfontein, Richtersveld, $28.41^{\circ} \mathrm{S}, 16.88^{\circ} \mathrm{E}, 20 .-23 . x i .1975$, V.B. Whitehead, SAMC; 1 \% , Clanwilliam, $32.18^{\circ} \mathrm{S}, 18.88^{\circ} \mathrm{E}$, ix.1977, V.B. Whitehead, SAMC; $10^{\top}$, Richtersveld N Park, Paradise Kloof, $28.19^{\circ} \mathrm{S}$, $17.01^{\circ}$ E, 22.ix.1995, F. W. Gess, on Drosanthemum sp., SAMC.

Etymology. This species' name refers to its yellow clypeus. 
Distribution (Fig. 98). This species is known only from the north-western region of South Africa.

Host plant (1 specimen). Aizoaceae, Drosanthemum sp. (1ठ).

First - last observations. Unspecified date in September to 23.xi.

\section{Capicola flavitarsis Friese, 1912}

Capicola flavitarsis Friese, 1912: 185, $\widehat{0}$.

Hesperapis flavitarsis (Friese): Cockerell, 1934: 446.

Capicola (Capicola) flavitarsis Friese: Michener, 1981: 82-83.

Capicola (Rhinochaetula) cinctiventris Friese, 1912: 186, ㅇ.

Syn. n.

Capicola cinctiventris Friese: Cockerell, 1915: 342-343.

Hesperapis (Capicola) cinctiventris (Friese): Eardley \& Urban, 2006: 164 .

Type material of $C$. flavitarsis (designated here). Lectotype $\widehat{0}$, South Africa, Willowmore $\left(33.28^{\circ} \mathrm{S}, 23.48^{\circ} \mathrm{E}\right)$, ZMHB Original designation: "Willowmore, Südafrika". Lectotype: $\delta$ $(\mathrm{ZMBH})$, labels: $1^{\text {st }}$ printed on white paper "Willowmore Capland Dr. Brauns."; $2^{\text {nd }}$ handwritten with black ink on white paper "Capicola flavitarsis 1910 Friese det."; $3^{\text {rd }}$ printed on red paper "Typus"; $4^{\text {th }}$ printed on red paper "Capicola flavitarsis Friese 1912 paralectotype design Michez 2006". Paralectotype: o (ZMBH), labels: $1^{\text {st }}$ printed on white paper "Willowmore Capland Dr. Brauns."; $2^{\text {nd }}$ handwritten with black ink on white paper "Rophites alfkeni Br."; $3^{\text {rd }}$ printed on red paper "Typus"; $4^{\text {th }}$ printed on white paper "Coll. Friese"; $5^{\text {th }}$ printed on white paper "Zool. Mus. Berlin"; $6^{\text {th }}$ printed on red paper "Capicola flavitarsis Friese 1912 lectotype design Michez 2006".

Type material of $C$. cinctiventris (designated here). Lectotype + , South Africa, Ookiep $\left(29.6^{\circ} \mathrm{S}, 17.9^{\circ} \mathrm{E}\right)$, ZMHB. Original designation: "Ookiep, Südafrika". Lectotype: o $(\mathrm{ZMBH})$, labels: $1^{\text {st }}$ printed on white paper "Bushmanld Jackals Water Lightfoot"; $2^{\text {nd }}$ handwritten with black ink on white paper "Rhinochaetula cinctiventris 1910 Friese det."; $3^{\text {rd }}$ printed on red paper "Typus"; $4^{\text {th }}$ printed on white paper "Coll. Friese"; $5^{\text {th }}$ printed on red paper "Capicola cinctiventris Friese 1912 lectotype design Michez 2006"; $6^{\text {th }}$ printed on white paper "Capicola flavitarsis det. Michez".

The synonymy is based on female of $C$. cinctiventris and male of $C$. flavitarsis collected at the same time $(4 \% / 10$, Namibia, Karasburg $\left(28.02^{\circ} \mathrm{S}, 18.75^{\circ} \mathrm{E}\right), 22 . x .1968$, J.G. Rozen, AMNH). Moreover both present same structure of proboscides and propodeum (unsexual features).

Diagnosis (Figs 30-37). $q$ and $\hat{\sigma}$. Integument mainly black. Head wider than long. Maxillary palpus longer than labial palpus, as long as galea. Outer surface of galea mat and sculptured. Clypeus about two and a half times as wide as long. Compound eyes converging a little ventrally. Mesoscutum and scutellum shiny and nearly hairless. Basal half of propodeal triangle punctulate to strigate/strigulate. Propodeum shiny. + (Fig. 30). L = 7.2 $\mathrm{mm}$. Labrum, base of mandibles, apex of the clypeus, legs and basal part of metasoma mostly reddish. Clypeus shiny and densely punctate $(\mathrm{i}=\mathrm{d})$. Legs $1-3$ with yellowish hairs. Spurs of Tb2 with 4-6 sharp outstanding spines. T1-T3 black and shiny. T1 with interrupted apical fringe. Prepygidial fimbria yellowish. Pp with a strong elevated median area; not tapering (Fig. 30); weakly concave medially. ô (Figs 31-37). L = $6.0 \mathrm{~mm}$. Clypeus without medio-longitudinal groove. Clypeus and face covered with appressed white hairs. Eyes strongly prominent (Fig.
34). A5 as wide as long. A4-13 dorsally brown, ventrally orangish. Scutellum shiny with prominent, sparse punctures $(\mathrm{i}>3 \mathrm{~d})$. Tb2-3 and tarsi $1-3$ reddish. Tb3 apically weakly broadened (Fig. 31). S6 median part ended in short spiny processes (Fig. 32); medio-ventral process well developed (Fig. 32). S7 apex narrowed (Fig. 33). Apical area of S8 subtrianglar. Genitalia as illustrated in Figs 35-37.

Additional material examined (24 specimens). Namibia: $4 \% / 1 \delta^{\circ}$, Karasburg $\left(28.02^{\circ} \mathrm{S}, 18.75^{\circ} \mathrm{E}\right), 22 . x .1968$, J.G. Rozen, AMNH; $1 \delta^{\star}$, Warmbad, Kaokoveld, $19.17^{\circ} \mathrm{S}, 13.82^{\circ} \mathrm{E}$, ii.1925, Museum Expedition, SAMC. South Africa: $2 \hat{0}$, Touwsrivier $\left(33.33^{\circ} \mathrm{S}, 20.03^{\circ} \mathrm{E}\right), 08 . x i .1968$, J.G. Rozen, AMNH; $10^{\star}$, Mesklip $\left(29.83^{\circ} \mathrm{S}, 17.85^{\circ} \mathrm{E}\right), 21 . x .1968$, J.G. Rozen, AMNH; 1 \&/3 $0^{\circ}, \quad$ Touwsrivier $\quad\left(33.33^{\circ} \mathrm{S}, \quad 20.03^{\circ} \mathrm{E}\right), \quad 08 . x i i .1996$, Carpenter, AMNH; 2 \% , Vanrhynsdorp $\left(31.07^{\circ} \mathrm{S}, 18.55^{\circ} \mathrm{E}\right)$, 04.x.2003, Timmerman, UM; $2 \uparrow / 2 \delta^{\star}$, Hester Malan Nature Reserve, $29.66^{\circ} \mathrm{S}, 17.98^{\circ} \mathrm{E}, 17 . x .1987$, M. Struck, SAMC; $10^{\star}$, Moordenaars Karoo $\left(32.82^{\circ} \mathrm{S} 21.5^{\circ} \mathrm{E}\right)$, x.1952, Swanepoel, SAMC; $2{ }^{\star}$, Dikbome, Merweville Koup $\left(32.67^{\circ} \mathrm{S} 21.51^{\circ} \mathrm{E}\right)$, x.1952, Museum Expedition, SAMC; 1 $q$, Bushmanland, Jackals Water $\left(30.6^{\circ} \mathrm{S}, 21.15^{\circ} \mathrm{E}\right), \mathrm{x} .1911$, Lightfoot, SAMC; $10^{\star}$, Moordenaars Karoo $\left(32^{\circ} \mathrm{S}, 21.31^{\circ} \mathrm{E}\right)$, x.1952, Swanepoel, SAMC.

Distribution (Fig. 94). Western regions of South Africa and Namibia.

Host plant. Unknown.

First - last observations. 17.x - unspecified date in February.

\section{Capicola gessorum Eardley sp. $n$.}

Diagnosis (Figs 38-43). $q$ and $\delta$. Head wider than long, vertex gently convex in frontal view. Labial palpus two-thirds as long as maxillary palpus, reaching to apex of glossa. Maxillary palpus a little longer than galea. Outer surface of galea smooth. Compound eyes slightly converging ventrally. Clypeus two and a half times as wide as long, with medio-longitudinal groove, densely punctate $(\mathrm{i}=\mathrm{d})$. + . $\mathrm{L}=5.8 \mathrm{~mm}$. Clypeus, mesoscutum and scutellum shiny (some specimens with short appressed hairs). Propodeal triangle mostly shiny. Propodeum shiny. Legs 1-3 with white or pale yellow hairs. Spurs of Tb2 with tiny spines. Scopa sparsely pubescent. Apical hair band on T1 interrupted medially to continuous. T1-T5 reddish. T2-T4 with brown spot on foveae. Prepygidial fimbria yellowish. Pp with a pair of median, subparallel carinae, proximal region in area between these carinae narrow and weakly depressed mediolongitudinally; tapering to apex. $\widehat{\delta}$ (Figs $38-43$ ). $\mathrm{L}=3.8 \mathrm{~mm}$. Integument blackish, including antennae and legs. Eyes not prominent. Scutum and terga deeply punctate. Scutellum shiny, moderately punctate $(i=d)$. Tb3 with inner apical tooth (Fig. 43). Disc of T2-4 with basal concavity. S6 with medio-apical curved blade (Fig. 38). S7 disc very large (Fig. 39). S8 with short column; apical plate flattened (Fig. 40). Genitalia as illustrated in Figs 41-43.

Description. 9 . Head. $\mathrm{L}=1.6 \mathrm{~mm} ; \mathrm{W}=2.2 \mathrm{~mm}$. Head wider than long. Integument mostly black. Mandibles base, and antennal flagellum orange. Clypeus, face, genal area and preoccipital ridge with white, erect hairs, vertex with yellowish appressed hairs. Face shiny. Labial palpus 

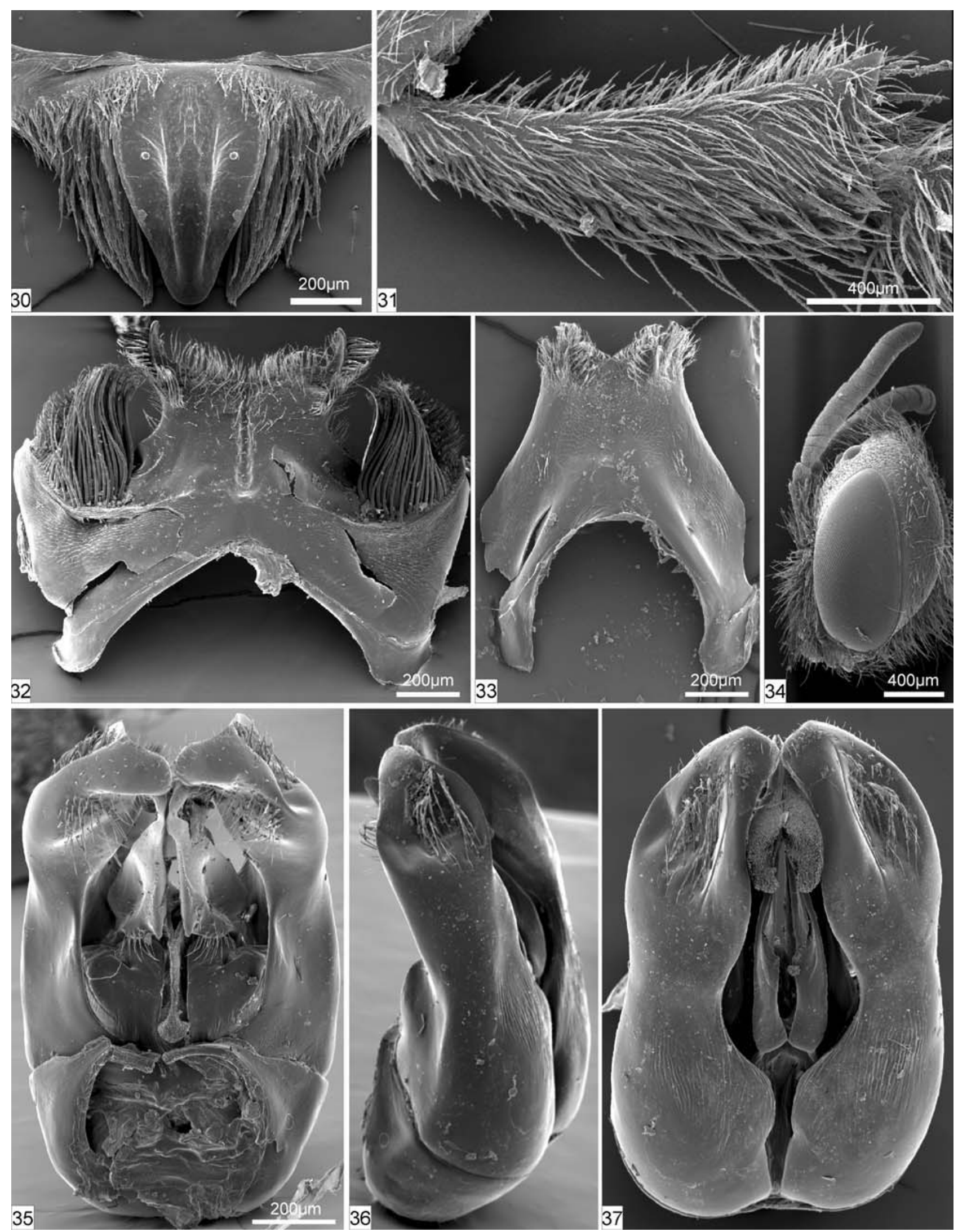

Figs 30-37. Capicola flavitarsis. 30 - dorsal view of pygidial plate $\$ ; 31$ - outer view of hind tibia $\delta ; 32$ - ventral view of sternum $6 \hat{\delta} ; 33$ - ventral view of sternum $7 \hat{\delta} ; 34$ - lateral view of head $\delta ; 35$ - ventral view of genitalia $\delta^{\star} ; 36$ - lateral view of genitalia $\hat{\sigma}$ (scale as in Fig. 35); 37 - dorsal view of genitalia $\hat{\sigma}$ (scale as in Fig. 35). 

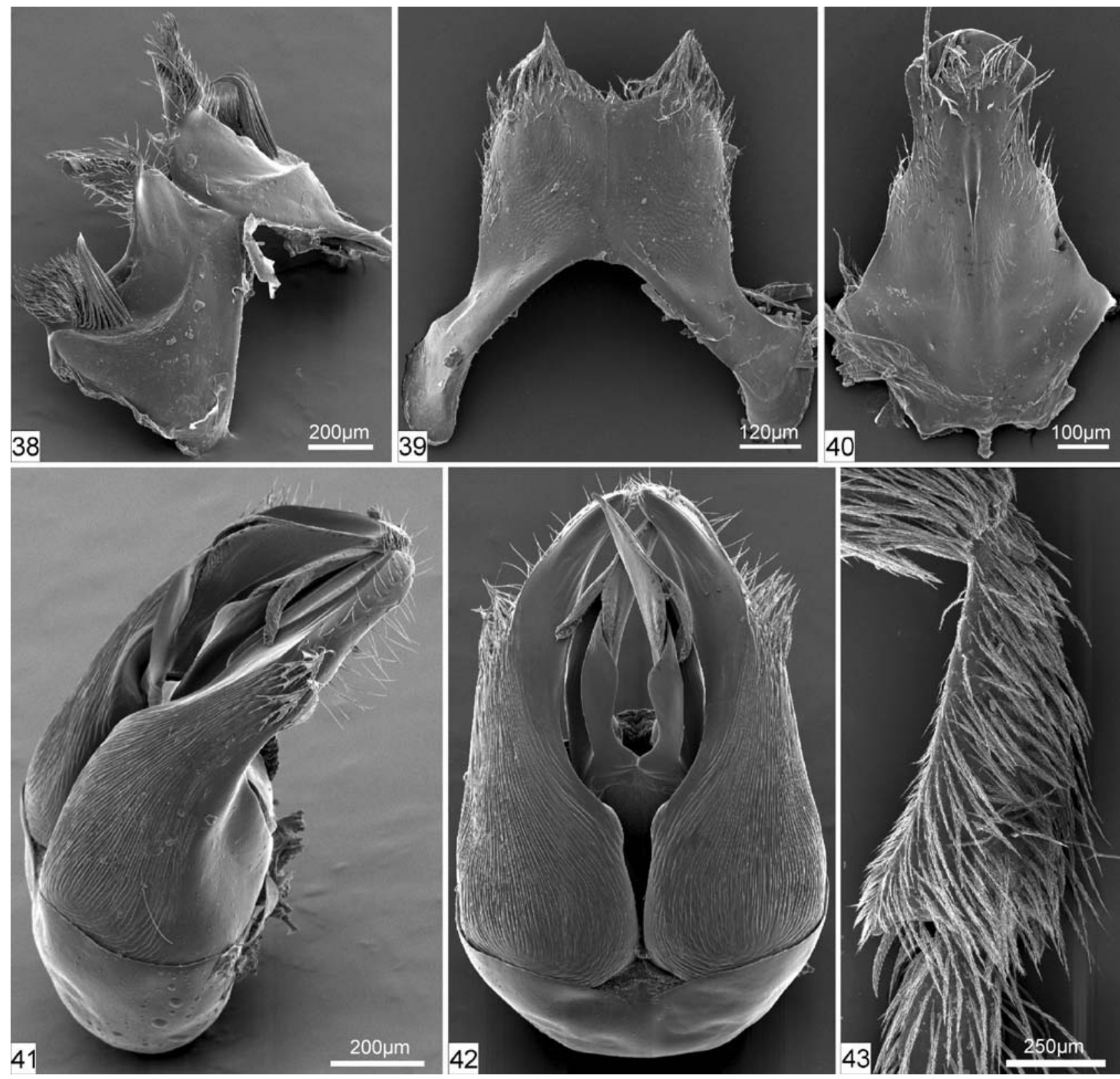

Figs 38-43. Capicola gessorum sp. n., đ. 38 - lateral view of sternum 6;39 - ventral view of sternum 7; 40 - ventral view of sternum 8; 41 - lateral view of genitalia; 42 - dorsal view of genitalia (scale as in Fig. 41); 43 - outer surface of hind tibia .

two-thirds as long as maxillary palpus, reaching beyond apex of glossa. Maxillary palpus a little longer than galea. Galea shiny. Malar area shorter than A2. Face and vertex densely, and distinctly punctate. Clypeus two and a half times as wide as long; with few deep punctures, with slight mediolongitudinal groove. Compound eyes converging slightly ventrally. Antennal flagellum orange. Mesosoma. $\mathrm{L}=1.1 \mathrm{~mm}$. $\mathrm{W}=1.6 \mathrm{~mm}$. Integument black. Mesoscutum and scutellum shiny, with some short appressed hairs, densely but weakly punctate $(i<d)$. Mesepisterna and metanotum with long, erect, white hairs. Propodeal triangle hairless, mostly glabrous. Propodeum shiny between dense punctures. Legs. Integument of leg 1, F2, Tb2 and F3 reddish-black; tarsi 2-3 and Tb3 yellowish. Hairs mostly pale yellowish, scopa sparse. Spurs of Tb2 with about ten tiny spines. Tb3 and Bt3 with red spots at the hair base. Wings. Surface transparent. Two submarginal cells, the first longer than the second. Basal vein slightly curved. Metasoma. $\mathrm{L}=3.5 \mathrm{~mm} . \mathrm{W}=$ $1.0 \mathrm{~mm}$. Terga and sterna reddish. Discs of terga shiny, with weak punctures. T1 apical hair band continuous. T2-T4 with white continous apical hair bands. T2-T4 with brown spot on lateral margin. Prepygidial fimbria yellowish. Sterna shiny. Sterna and terga with straight premarginal line. Pp with broad pair of median, subparallel carinae, area between these carinae with narrow, weak depression basally; tapering to apex; base reddish; apex red. $\delta$. Head. $\mathrm{L}=1.4 \mathrm{~mm}$. $\mathrm{W}=2.0 \mathrm{~mm}$. Head wider than long. Integument black, reddish-black in places. Face, genal area and preoccipital ridge with white hairs. Vertex shiny, with fine, dense punctation. Maxillary palpus less than two-thirds as long as labial palpus, the 
latter reaching end of glossa. Maxillary palpus as long as galea, which is a little longer than labial palpus. Galea shiny. Labrum not swollen. Malar area shorter than A2. Clypeus two and a half times as wide as long, densely punctate $(i<d)$, with a mediolongitudinal groove. Compound eyes ventrally converging. Face and vertex with small, shallow puncture. A3 longer than A4. Mesosoma. $\mathrm{L}=1.6 \mathrm{~mm} . \mathrm{W}=1.1 \mathrm{~mm}$. Integument of scutum, scutellum, propodeum, fore and middle legs black. Vestiture generally long and white. Mesoscutum, scutellum, metanotum and propodeum with small, shallow, fairly sparse punctures $(i<d)$. Anterior part of propodeal triangle shiny, with a little rugose sculpture, posterior part glabrous. Legs. Sculpture reticulate. Vestiture white, except ventral surfaces of tarsi very pale yellow. Tb3 with apical tooth. Wings. Transparent. Two submarginal cells; first longer than second. Basal vein slightly curved. Metasoma. $\mathrm{L}=2.4 \mathrm{~mm} . \mathrm{W}=1.2 \mathrm{~mm}$. Integument $\mathrm{T} 1-\mathrm{T} 4$ and S1-S4 black. T1-T4 with white, medially interrupted, apical fringes. Terga densely and deeply punctate. S6 with medio-apical curved blade (Fig. 38). S7 disc very large (Fig. 39). S8 with short column (Fig. 40); apical plate flattened. Genitalia as illustrated in Figs 41-43.

Type material (16 specimens). Holotype $\delta, 3$ paratypes $q$, 12 paratypes $\delta$. South Africa: male holotype, $2 \% / 100^{t}$, Voëlklip (Namaqualand), $29.45^{\circ} \mathrm{S}, 17.22^{\circ} \mathrm{E}, 2 .-7 . x .1994$, F.W \& S.K. Gess, on Campanulaceae (pinkish violet), AMGS; $1 \uparrow / 2 \widehat{0}$, idem, on Whalenbergia cf. annularis; 19 , idem, 28.ix.1997, on Lightfootia namaquana.

Etymology. Named after Sarah and Fred Gess who collected the type material.

Distribution (Fig. 94). Only known from the locus typicus, Voëlklip (South Africa, Namaqualand).

Host plant (13 specimens). Campanulaceae, pinkish violet $\left(2 \% / 1 \delta^{\star}\right)$, Wahlenbergia sp. (9 $\left.{ }^{\star}\right)$ and Lightfootia namaquana (1ㅇ).

First - last observations. 28.ix.-07.x.

\section{Capicola micheneri Michez sp. $n$.}

Diagnosis (Figs 44-57). $q$ and $\hat{\sigma}$. Head wider than long. Maxillary palpus longer than galea. Outer surface of galea sculptured and mat. Clypeus three times as wide as long, without median groove. Compound eyes slightly converging ventrally. Propodeal triangle rugose, with horizontal basal area (Fig. 44). + (Figs 45-51). L $=5.8$ $\mathrm{mm}$. Metasoma mostly reddish. Spurs of Tb2 with $6-8$ sharp outstanding spines (Fig. 47). Outer face of Tb3 with few large, blunt, spine-like hairs (Fig. 46). T2-T4 with black lateral spots. Prepygidial fimbria brown. Pp with a strong median carina (Figs 48-49). ơ (Figs 52-57). L = $4.5 \mathrm{~mm}$. Body mainly black. Antennae ventrally yellowish, dorsally brown. A5 as wide as long. Scutum shiny and densely punctate $(\mathrm{i}=\mathrm{d})$. Tb3 not expanded. Terga with continous apical fringes. S6 with large medio-apical teeth nearly as long as length of disc (Fig. 52), without medio-longitudinal groove. S7 with apical lobes diverging (Fig. 53). S8 with large median carina (Fig. 54); column curved. Genitalia as illustrated in Figs 55-57.

Description ․ Head. $\mathrm{L}=1.5 \mathrm{~mm} . \mathrm{W}=2.1 \mathrm{~mm}$. Head wider than long; mainly black. Mandible base reddish; apex red. Labrum and apex of clypeus reddish. Scape black. Flagellum ventrally reddish or yellow, dorsally brownish. Clypeus, face and genal area with white, erect hairs. Vertex shiny. Labial palpus as long as glossa; shorter than maxillary palpus. Maxillary palpus longer than galea. Outer surface of galea scluptured and mat. Malar area shorter than A2. Clypeus glabrous; three times as wide as long; with few deep punctures ( $\mathrm{i}>3 \mathrm{~d})$, denser at base. Compound eyes slightly converging below. Flagellum twice as long as scape. Mesosoma. $\mathrm{L}=1.8 \mathrm{~mm}$. $\mathrm{W}=1.5 \mathrm{~mm}$. Integument black. Mesoscutum, scutellum and mesepisterna with a few short whitish hairs. Metanotum covered with short, whitish hairs. Mesoscutum, scutellum and metanotum shiny, with dense, weak punctures, more than a puncture width apart. Propodeal triangle hairless, mat and rugose; with horizontal basal area (Fig. 44). Legs. Tarsi $1-3$ and Tb2-3 reddish. F1-3 and $\mathrm{T} 1$ brownish. Spurs of Tb2 with $8-10$ sharp outstanding spines (Fig. 47). Legs 1 and 3 with sparse white hairs. Leg 2 covered with appressed white hairs. Inner surface of $\mathrm{Tb} 3$ with keirotrichia (Fig. 45). Outer face of Tb3 with few large, blunt, spine-like hairs (Fig. 46). Wings. Surface hyaline. Two submarginal cells, first longer than second. Basal vein slightly curved. Metasoma. $\mathrm{L}=3.3 \mathrm{~mm}$. $\mathrm{W}=1.5 \mathrm{~mm}$. Metasoma mainly reddish. T2-T4 with black lateral spots. Pp reddish at base, red at apex. Disc of terga and sterna shiny. T1 with reduced white apical fringe. T2-T4 with white apical fringe. Prepygidial fimbria brown. Terga and sterna with weak punctures. Terga and S4-S5 with straight apical margin. S2-S3 with emarginated apex. Pp with median area strongly elevated (Figs 48-49). $\tau$. Head. L $=1.2$ $\mathrm{mm}$. $\mathrm{W}=1.8 \mathrm{~mm}$. Head wider than long; mainly black. Mandible with base reddish, apex red. Labrum reddish. Flagellum ventrally yellowish, dorsally brown. Clypeus, face and genal area covered with white, appressed hairs. Vertex glabrous. Labial palpus as long as glossa; shorter than maxillary palpus. Maxillary palpus longer than galea. Outer surface of galea sculptured and mat. Malar area shorter than A2. Clypeus shiny; three times as wide as long; with deep punctures, denser at base. Compound eyes slightly converging. Flagellum three times as long as scape. Mesosoma. $\mathrm{L}=1.5 \mathrm{~mm}$. $\mathrm{W}=1.2 \mathrm{~mm}$. Integument black. Mesoscutum, scutellum and mesepisterna with short whitish hairs. Metanotum covered with short whitish hairs. Mesoscutum, scutellum and metanotum shiny, with dense weak punctures $(i=d)$. Propodeal triangle hairless, mat and rugose. Legs. Integument mainly black to brown; with few long, white, erect hairs. Mediotarsi 1-3 and hind tarsi 1-3 reddish. Tb3 untoothed; with keirotrichia on inner face. Wings. Surface transparent. Two submarginal cells, the first longer than the second. Basal vein slightly curved. Metasoma. $\mathrm{L}=2.4 \mathrm{~mm} . \mathrm{W}=$ $1.5 \mathrm{~mm}$. Terga and sterna black. Disc of terga glabrous. T1-T6 with long white, apical fringes. Disc of sterna glabrous. Sterna with few apical hairs. Terga and sterna with weak punctures. Terga and S1-S5 with straight apex. S6 with medio-apical teeth as long as length of disc (Fig. 52). S7 with apical lobes diverging, with disc 

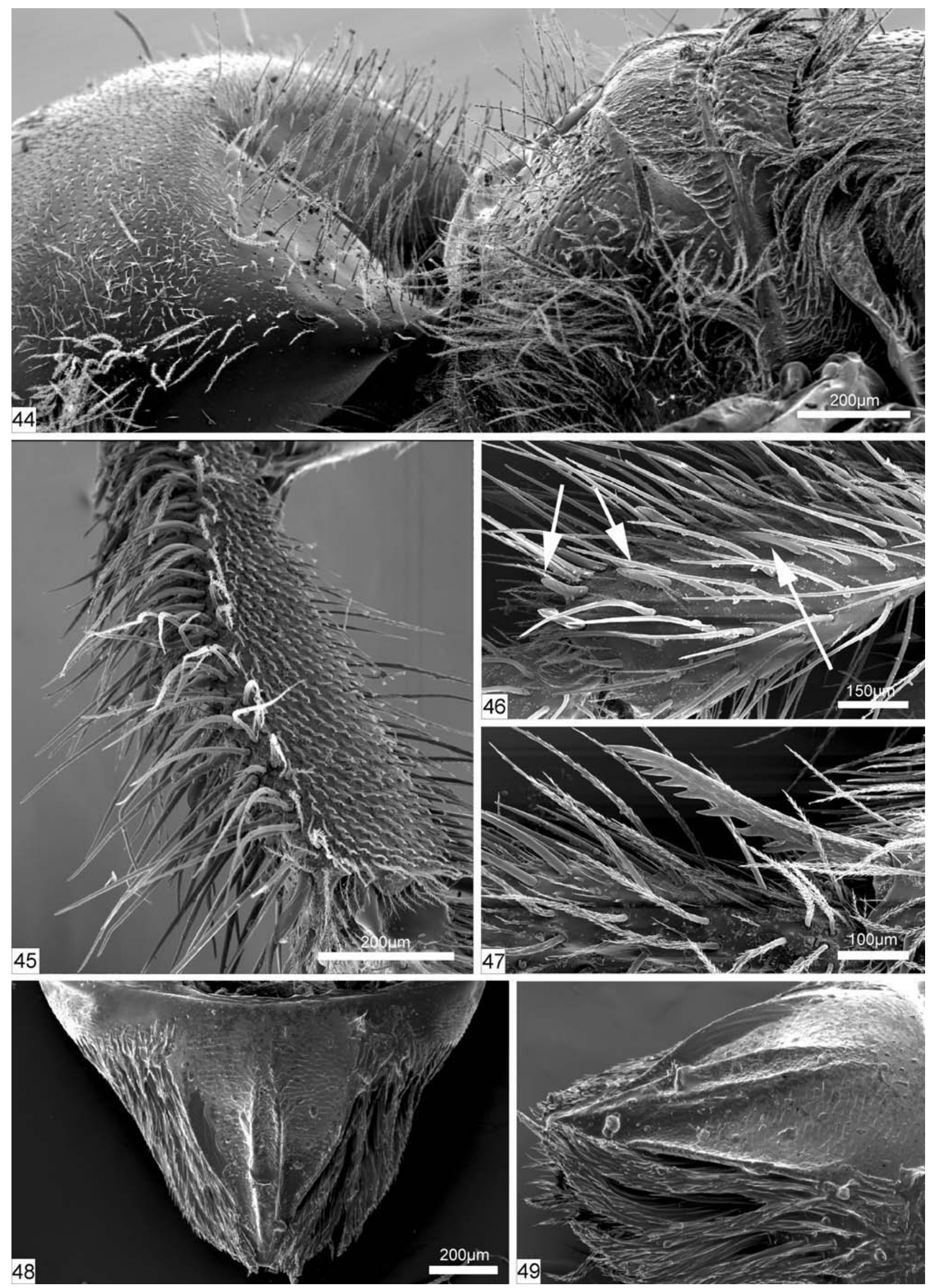

Figs 44-49. Capicola micheneri, ‥ 44 - lateral view of propodeum; 45 - inner surface of Tb3; 46 - outer surface of scopa, showing large, blunt, spine-like hairs (arrows); 47 - Spur of Tb2. 48 - dorsal view of pygidial plate; 49 - lateral view of pygidial plate (scale as in Fig. 48). 
reduced (Fig. 53). S8 with large median carina (Fig. 54); column curved. Genitalia as illustrated in Figs 55-57.

Type material (29 specimens). Holotype $\widehat{\delta} ; 8$ paratypes $\widehat{0}$; 20 paratypes $\%$; Namibia, Usakos $\left(22^{\circ} \mathrm{S}, 15.6^{\circ} \mathrm{E}\right)$; Original labelling: South Africa: 46 km W. Usakos March 21, 1976, J.G. \& B.L. J.G. Rozen, collected on Indigofera sp.; type depository: AMNH.

Additional material examined (46 specimens). Namibia: 3 \%, $29 \mathrm{~km}$ ESE Seeis $\left(22.45^{\circ} \mathrm{S}, 17.58^{\circ} \mathrm{E}\right), 16.1 i .1976$, J.G Rozen, AMNH; 1 \&, 9 km ESE Seeis, 12.iii.1976, J.G. Rozen, AMNH; 10, 20 km ESE Seeis, 13.iii.1976, J.G. Rozen, AMNH; 1 , $22 \mathrm{~km}$ ESE Seeis, 15.iii.1976, J.G. Rozen, AMNH; 2 우, Kuiseb River, $50 \mathrm{~km}$ SE Rooibank $\left(23.18^{\circ} \mathrm{S}, 14.65^{\circ} \mathrm{E}\right)$, 20.iii.1976, J.G. Rozen, AMNH; $79 / 7$ §, 78 km E Swakopmund $\left(22.68^{\circ} \mathrm{S}, 14.53^{\circ} \mathrm{E}\right), 21$. iii.1976, J.G. Rozen, on Indigofera $\mathrm{sp}$., AMNH; 6 ㅇ, Uis/Henties Bay, $21.27^{\circ} \mathrm{S}, 14.45^{\circ} \mathrm{E}, 17.1 \mathrm{iv} .2002$, F.W. \& S.K. Gess, on pink Indigofera sp., AMGS; 1 \%, idem, SANC; 9요 Swakopmund/Usakos, Near Rossing Mountain, $22.34^{\circ} \mathrm{S}, 14.49^{\circ} \mathrm{E}, 15 .-28 . i v .2002$, F.W. \& S.K. Gess, on pink Indigofera sp., AMGS; 19 , idem, SANC; 29 , SE Bullsport, 4 $\mathrm{km}$ from $\mathrm{C} 14$ on $\mathrm{D} 854,24.11^{\circ} \mathrm{S}, 16.24^{\circ} \mathrm{E}, 11 . i i i .2000, \mathrm{~F} . \mathrm{W} . \&$ S.K. Gess, on white Limeum sulcatum, AMGS; $2 \uparrow / 1 \delta^{\star}, 2 \mathrm{~km}$ from $\mathrm{C} 17$ on $\mathrm{R} 511$ road to Mata Mata, $25.37^{\circ} \mathrm{S}, 19.25^{\circ} \mathrm{E}$, 7.iii.2000, F.W. \& S.K. Gess, on semi-prostrate Indigofera sp., AMGS; 1 ㅇ, C17 Koes/Gochas, $25.39^{\circ} \mathrm{S}, 19.24^{\circ} \mathrm{E}$, 7.iii.2000, F.W. \& S.K. Gess, on Indigofera sp., AMGS; 1 , Klein-Aus Vista $\left(26.65^{\circ} \mathrm{S}, 16.25^{\circ} \mathrm{E}\right), 2$.iii.2000, F.W. \& S.K. Gess, AMGS.

Etymology. Named after Prof. C.D. Michener who is an early reviewer of Melittidae.

Distribution (Fig. 95). Widespread through the central, summer rainfall areas of Namibia. This species' close relative, C. aliciae, is only known from the winter rainfall areas of Namibia and from winter and su mmer rainfall areas in South Africa.

Host plant (66 specimens). Fabaceae, Indigofera sp. (47\%/17ð); Molluginaceae, Limeum sulcatum (2ㅇ).

First - last observations. 12.ii.-28.iv.

\section{Capicola nanula Cockerell, 1936}

Capicola nanula Cockerell, 1936b: 482, ô.

Capicola (Capicola) nanula (Cockerell): Michener, 1981: 81, 83.

Type material. Holotype $\hat{\sigma}$, South Africa, Beaufort West $\left(32.35^{\circ} \mathrm{S}, 22.58^{\circ} \mathrm{E}\right), \mathrm{NHMN}$. Original designation: "Beaufort West, Cape Province". Cockerell (1936b) described the male of C. nanula. We present here the first description of the female. The described females are associated to three samples with males: $12 \% / 160^{\circ}$, S.W. Africa, Okahandja $\left(21.98^{\circ} \mathrm{S}, 16.92^{\circ} \mathrm{E}\right)$, 3.-9.ii.1928, Turner, det. Cockerell, NHMN; 1 \%/4 0 , South Africa, Cape Province, $16 \mathrm{~km} \mathrm{~S}$. of Vioolsdrif $\left(28.77^{\circ} \mathrm{S}\right.$,

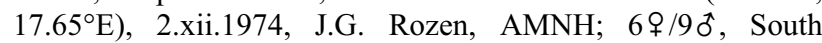
Africa, Cape Province, $16 \mathrm{~km} \mathrm{~S}$. of Vioolsdrif, 29.xi.1974, on Trianthema cristallina (Aizoaceae), J.G. Rozen, AMNH. The described specimens match well with Cockerell's description of males, thus their description as the unknown sex of $C$. nanula.

Diagnosis (Figs 58-63). $q$ and $\delta$. Head wider than long. Vertex flat in frontal view. Labial palpus threefourths as long as maxillary palpus, reaching beyond apex of glossa. Maxillary palpus twice as long as galea. Outer surface of galea sculptured and mat. Compound eyes slightly converging ventrally. Clypeus slightly more than three times as wide as long, with few punctures ( $\mathrm{i}>3 \mathrm{~d}$ ). Propodeal triangle rugose, with horizontal basal area.
Propodeum mat, with horizontal basal area. $q$ (Figs 58-59). $\mathrm{L}=5.8 \mathrm{~mm}$. Clypeus, mesoscutum and scutellum shiny (some specimens with short appressed hairs). Legs 1-3 with white to pale yellow hairs. Spurs of Tb2 without large spine. Scopa sparsely pubescent. Apical hair band on T1 medially interrupted to continuous. T1-T5 reddish. T2-T4 with brown spot on foveae. Prepygidial fimbria yellowish. Pp with median subparallel carinae, proximal region of carinae narrow and weakly depressed (Figs 58-59). ô (Figs 60-63). L = $3.8 \mathrm{~mm}$. Integument mostly black. Eyes not prominent. A4-10 yellow, A11-13 dark. Scutellum shiny, sparsely punctate. F1-3 black. Tb1-3 and Bt1-3 yellow. Tb3 not broadened, untoothed. S6 median part ended in short spiny processes (Fig. 60). S7 long, median width narrower than apical one (Fig. 61). Apical plate of S8 sub-triangular. S8 and genitalia as illustrated in Figs 62-63.

Description. ․ Head. $\mathrm{L}=1.3 \mathrm{~mm} ; \mathrm{W}=2.2 \mathrm{~mm}$. Head wider than long. Integument mostly black. Mandibles base, labrum and clypeus yellow to reddish. Clypeus, face, genal area, vertex and preoccipital ridge with yellow or orange appressed hairs. Face shiny. Labial palpus three-fourths as long as maxillary palpus, reaching beyond apex of glossa. Maxillary palpus twice as long as galea. Outer surface of galea sculptured. Malar area shorter than A2. Face and vertex densely, but weakly punctate. Clypeus three times as wide as long; with few deep punctures and a medio-longitudinal groove. Compound eyes converging ventrally. Antennae ventrally reddish, dorsally brownish to entirely yellow. Mesosoma. L $=1.5 \mathrm{~mm}$. $\mathrm{W}=1.1 \mathrm{~mm}$. Integument black. Mesoscutum and scutellum shiny (some specimens with short appressed hairs), densely but weakly punctate, punctures more than width puncture apart. Mesepisterna and metanotum with long, erect, yellowish hairs. Propodeal triangle hairless, entirely rugose, with basal area horizontal. Propodeum mat. Legs. Integument yellow to reddish. Fore legs with yellowish hairs. Inner surface of mid legs with yellow hairs. Outer side of mid legs with long white appressed hairs. Hind legs with yellowish hairs, scopa sparse. Spurs of $\mathrm{Tb} 2$ with about six small spines. Tb3 and Bt3 with red spots at the hair base. Wings. Surface transparent. Two submarginal cells, the first longer than the second. Basal vein slightly curved. Metasoma. $\mathrm{L}=2.7$ $\mathrm{mm} . \mathrm{W}=1.4 \mathrm{~mm}$. Terga and sterna reddish. Discs of terga shiny, with weak punctures. T1 apical hair band interrupted medially to continuous. T2-T4 with white continuous apical hair bands. T2-T4 with brown spot on lateral margin. Prepygidial fimbria yellowish. Sterna shiny. Sterna and terga with straight premarginal line. Pp with median subparallel carinae, proximal region of carinae narrow and weakly depressed (Figs 58-59); base reddish; apex red.

Additional material examined (94 specimens). Namibia: 12 $/ 160^{\circ}$, Okahandja $\left(21.98^{\circ} \mathrm{S}, 16.92^{\circ} \mathrm{E}\right), 10 .-16 . i i .1928$,

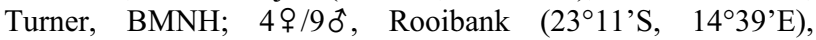

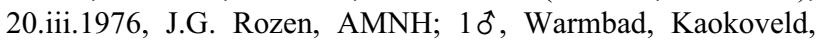
$19.17^{\circ} \mathrm{S}, 13.82^{\circ} \mathrm{E}$, ii. 1925 , Museum Expedition, SAMC. South

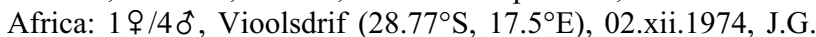
Rozen, AMNH; 6잉, idem, 29.xi.1974, on Trianthema 

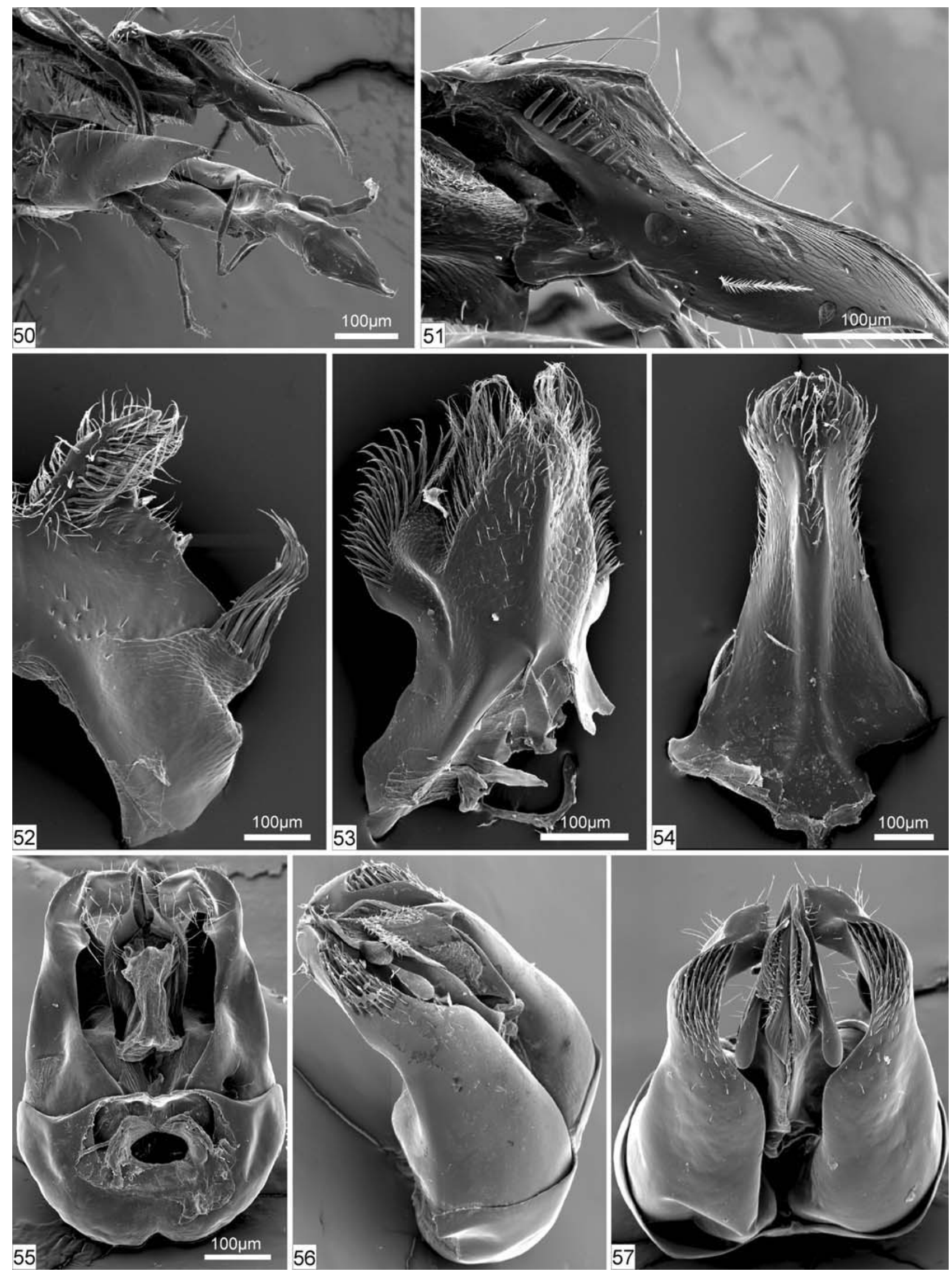

Figs 50-57. Capicola micheneri sp. n. 50 - mouthpart $q ; 51$ - inner surface of galea $\$$; 52 - ventral view of sternum $60 ; 53-$ lateral view of sternum $7 \delta^{\dagger} ; 54$ - ventral view of sternum $8 \delta^{\lambda} ; 55$ - ventral view of genitalia $\delta^{\lambda} ; 56$ - lateral view of genitalia $\hat{\sigma}$ (scale as in Fig. 55). 57 - dorsal view of genitalia $\hat{o}$ (scale as in Fig. 55). 

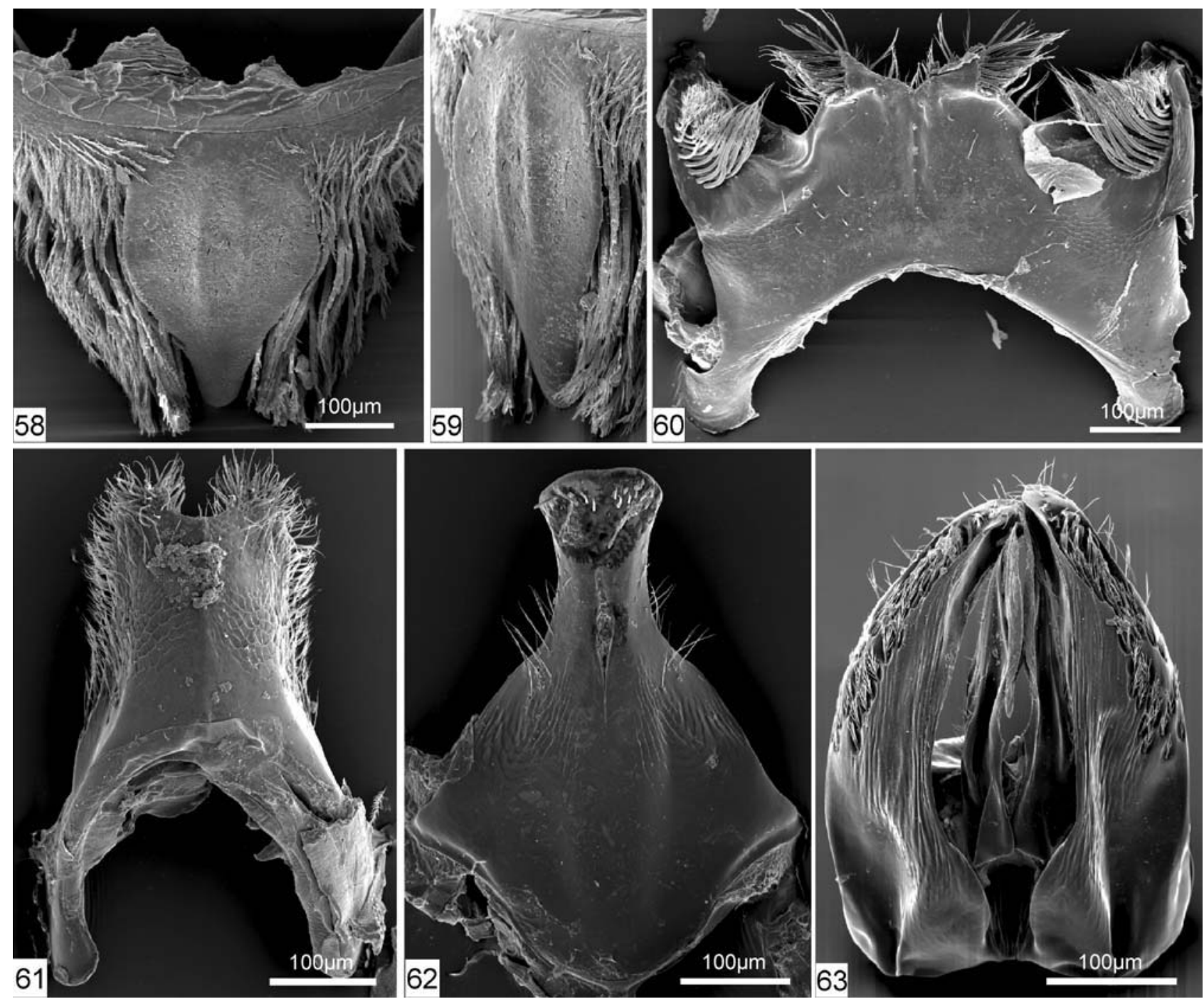

Figs 58-63. Capicola nanula. 58 - dorsal view of pygidial plate $\$ ; 59$ - lateral view of pygidial plate (scale as in Fig. 58); 60 ventral view of sternum $6 \delta^{\pi} ; 61$ - ventral view of sternum $7 \delta ; 62$ - ventral view of sternum $8 ; 63$ - dorsal view of genitalia.

cristallina; 20ิ, Prince Albert Road (32 $\left.59^{\prime} \mathrm{S}, 21^{\circ} 41^{\prime} \mathrm{E}\right)$,

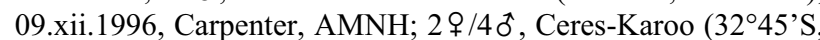
1950’E), 07.xii.1996, Carpenter, AMNH; 2 \% $/ 20^{\star}$, Vanhynsdorp (310's, $\left.18^{\circ} 56^{\prime} \mathrm{E}\right)$, 25.ix.1997, Kuhlmann, UM; 2 \%, idem, 04.x.2003, Timmerman, UM; 4ㅇ, $60 \mathrm{~km}$ Loriesfontein Kliprand $\left(30.6^{\circ} \mathrm{S}, 18.7^{\circ} \mathrm{E}\right)$, 31.x.1999, M. Halada, OOLL;

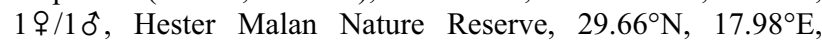
24.ix.1986, M. Struck, SAMC; 6 9 , Leliefontein, Paulshoek, $30.33^{\circ} \mathrm{S}, 18.26^{\circ} \mathrm{E}, 28 .-30 . i x .1997$, C. Seymour, SAMC; 5, Goegap Nature Reserve (Namaqualand), Kraaiwater, $29.38^{\circ} \mathrm{S}$, $18^{\circ} \mathrm{E}, 29 .-30 . i x .1997$, F.W. \& S.K. Gess, on Galenia sarcophylla, AMGS; 1 , Anenous, $29.14^{\circ} \mathrm{S}, 17.34^{\circ} \mathrm{E}, \mathrm{D} . \mathrm{W}$. Gess, on Wahlenbergia sp., AMGS.

Distribution (Fig. 98). This species is only known from southern Namibia and the western region of South Africa..

Host plant (21 specimens). Aizoaceae, Trianthema cristal-

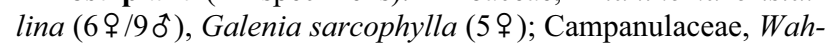
lenbergia sp. (1 9 ).

First - last observations. 24.ix.- $-16 . i i$.

\section{Capicola nigerrima Cockerell, 1932}

Capicola nigerrima Cockerell, 1932a: 456, ㅇ.
Hesperapis (Capicola) nigerrima (Cockerell): Eardley \& Urban, 2006: 164.

Type material. Holotype + , South Africa, Nieuwoudtville $\left(31.38^{\circ} \mathrm{S}, 19.1^{\circ} \mathrm{E}\right)$, NHMN. Original designation: "Nieuwoudtville, Cape Province". Cockerell (1932a) described only the female of $C$. nigerrima. We here give the first description for the male based on several pairs of specimens (see material examined below). These pairs females/males are morphologically very similar (cf. diagnosis) to the Cockerell species, which lead us to describe the unknown sex of $C$. nigerrima.

Diagnosis (Figs 1-4, 64-70). + and $\delta$. Integument black or brownish. Labial palpus shorter than glossa and maxillary palpus. Maxillary palpus as long as galea. Outer surface of galea smooth. Clypeus with a mediolongitudinal groove, two and a half times as wide as long. Clypeus, scutum, scutellum and disc of terga with deep, dense punctures $(\mathrm{i}<\mathrm{d})$. Propodeal triangle very short, with declivous basal area. + (habitus as in Figs 1-2). $\mathrm{L}=$ $8.9 \mathrm{~mm}$. Legs with white or pale yellow hairs. Spurs of Tb2 with 8-10 sharp outstanding spines. T1-T5 with white continous apical fringe. Prepygidial fimbria black 


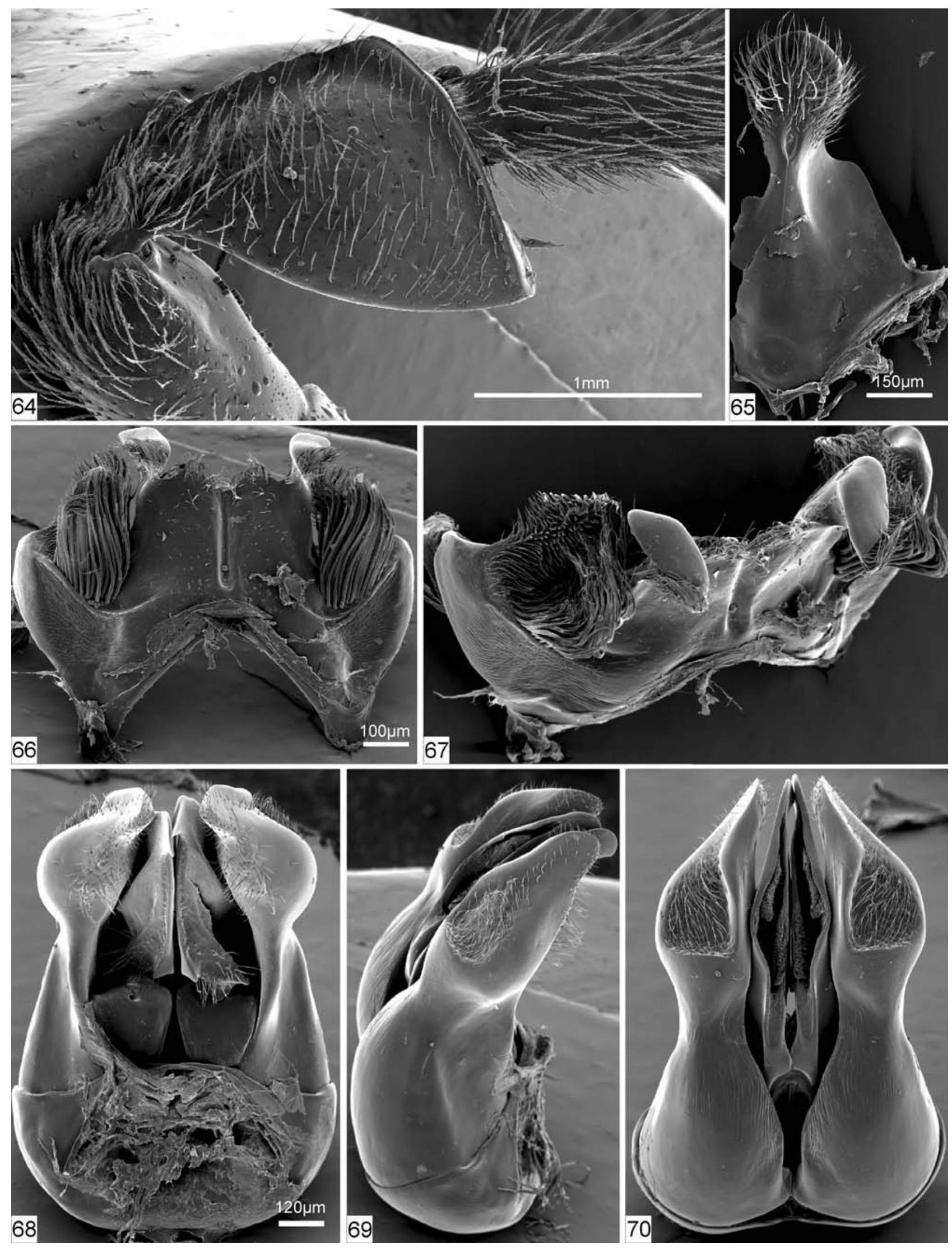

Figs 64-70. Capicola nigerrima, o $\widehat{0} .64$ - outer surface of hind tibia; 65 - ventral view of sternum 8; 66 - ventral view of sternum $6 ; 67$ - posterior view of sternum 6 (scale as in Fig. 66); 68 - ventral view of genitalia; 69 - lateral view of genitalia (scale as in Fig. 68 ); 70 - dorsal view of genitalia (scale as in Fig. 68). 
to brownish. Pp with a strong elevated median area. $\delta$ (habitus as in Figs 3-4; 64-70). L = 8.1 mm. Head wider than long. Inner margin of eyes converging ventrally. A4-13 brown. A5 longer than wide. Mesoscutum and scutellum shiny, with deep punctures. Tb3 strongly enlarged apically (Figs 4, 64). Disc of T2-4 deeply punctate, with basal concavity. Medio-apical processes of S6 curved and swollen (Figs 66-67). S7 disc very large. S8 apex spatulate (Fig. 65). Genitalia as illustrated in Figs 68-70.

Description. $\delta$. Head. $\mathrm{L}=2.7 \mathrm{~mm} . \mathrm{W}=3.3 \mathrm{~mm}$. Head wider than long. Integument black. Mandible reddish with black apex. Clypeus, face, genal area and preoccipital ridge with white, erect hairs. Vertex nearly hairless. Labial palpus shorter than glossa and maxillary palpus. Maxillary palpus as long as galea. Outer surface of galea smooth. Labrum swollen. Malar area shorter than A2. Clypeus trapezoidal, with dense and deep punctures $(i<$ d), with a medio-longitudinal groove. Compound eyes converging ventrally. Face and vertex deeply punctate. Antennae brown. A3 longer than A4. Mesosoma. L = 3.0 $\mathrm{mm} . \mathrm{W}=3.0 \mathrm{~mm}$. Integument black. Mesoscutum and scutellum with short, erect, brownish hairs on median area, and white, long, erect hairs on margins. Mesepisterna, metanotum and lateral part of propodeum with long, white, erect hairs. Mesoscutum, scutellum, metanotum and propodeum with deep and dense punctures $(\mathrm{i}<\mathrm{d})$. Anterior part of propodeal triangle declivous, transversally carinate. Legs. Integument brown. F1-3 nearly hairless. External face of Tb1-3 and tarsi 1-2 with white, erect hairs. External face of tarsus 3 with erect, brownish hairs. Inner side of tarsi 1-3 with long appressed yellow hairs. Inner side of Tb3 with keirotrichia. Apex of Tb3 strongly expanded (Fig. 64). Wings. Surface brown. Two submarginal cells; first longer than second. Basal vein slightly curved. Metasoma. $\mathrm{L}=4.2$ $\mathrm{mm}$. W = $3.3 \mathrm{~mm}$. Integument black. T1-T4 with white apical hair bands. S1-S5 nearly hairless. Disc of terga with deep, dense punctures $(i<d)$. Disc of sterna with dense, weak punctures. Apex of S1-S4 slightly emarginated. Apex of S5 notched. S6 with medio-apical process swollen; with a ventral process (Figs 66-67). S7 with very large body. S8 apex flattened (Fig. 65). Genitalia as illustrated in Figs 68-70.

Additional material examined (107 specimens). South Africa: $7 \delta^{\hat{\prime}}$, Velddrif $\left(32.78^{\circ} \mathrm{S}, 18.16^{\circ} \mathrm{E}\right)$, 15.x.1972, J.G. Rozen, R. McGinley \& C. Thompson, AMNH; 4ત, $28 \mathrm{~km}$ E. of Velddrif $\left(32.78^{\circ} \mathrm{S}, 18.16^{\circ} \mathrm{E}\right)$, 23.x.1972, J.G. Rozen, AMNH; 1 oै $^{\circ}$ Citrusdal $\left(32.6^{\circ} \mathrm{S}, 19.02^{\circ} \mathrm{E}\right), 02.1 x .1966$, J.G. Rozen, AMNH; $22 \% / 43 \sigma^{\circ}, 11 \mathrm{~km} \quad \mathrm{~W}$ Clanwilliam, $32.09^{\circ} \mathrm{S}, 18.45^{\circ} \mathrm{E}$, 2.-8.x.1990, F.W. \& S.K. Gess, on Wahlenbergia annularis, AMGS; $29,5 \mathrm{~km}$ W Clanwilliam $\left(32.18^{\circ} \mathrm{S}, 18.9^{\circ} \mathrm{E}\right)$, Graafwater road, 5.-6.x.1988, F.W. \& S.K. Gess, AMGS; 2 , , Die Berg, $32.10^{\circ}$ S, $18.43^{\circ}$ E, 4.x.1990, F.W. \& S.K. Gess, on Wahlenbergia annularis, AMGS; $2 \%, 10 \mathrm{~km}$ West Clanwilliam, Graafwater road, $32.10^{\circ} \mathrm{S}, 18.49^{\circ} \mathrm{E}, 4 . x .1990$, F.W. \& S.K. Gess, on Wahlenbergia annularis, AMGS; 19 , Raterfontein/ Olaf Bergfontein, $32.02^{\circ} \mathrm{S}, 18.35^{\circ} \mathrm{E}, 8 . x .1995$, F.W. \& S.K. Gess, on Wahlenbergia annularis, AMGS; $19 / 1$ o, $23 \mathrm{~km}$ from Citrusdal $\left(32.6^{\circ} \mathrm{S}, 19.02^{\circ} \mathrm{E}\right)$, on old road, 12.-13.x.1990, F.W. \& S.K. Gess, on Wahlenbergia annularis, AMGS; $1 \% / 1 \delta^{\dagger}$, between Nieuwoudtville and top of Vanrhyn's Pass $\left(31.38^{\circ} \mathrm{S}\right.$, $\left.19.02^{\circ} \mathrm{E}\right), 29 .-30 . i x .1990$, F.W. \& S.K. Gess, on Wahlenbergia annularis, AMGS; 5 \% , Citrusdal $\left(32.6^{\circ} \mathrm{S}, 19.02^{\circ} \mathrm{E}\right), 16 . x .1990$, F.W. \& S.K. Gess, on Wahlenbergia annularis, AMGS; 90, 5 $\mathrm{km}$ West Clanwilliam $\left(32.1^{\circ} \mathrm{S}, 18.49^{\circ} \mathrm{E}\right)$, on Graafwater road, 12.x.1987, F.W. \& S.K. Gess, AMGS; 1 \%, 14 km N. of Clanwilliam, $32.08^{\circ} \mathrm{S}, 18.75^{\circ} \mathrm{E}, 14 . x .1981$, V.B. Whitehead, SAMC; 1 \% $/ 1 \mathrm{o}^{\circ}$, Olywenboschkraal, $32.22^{\circ} \mathrm{S}, 18.82^{\circ} \mathrm{E}, 15 . \mathrm{x} .-9 . x i .2001$, V.B. Whitehead, SAMC; 2 \%, Paleisheuwel Station, $32.46^{\circ} \mathrm{S}$, $18.72^{\circ} \mathrm{E}$, 1.xi.2001, V.B. Whitehead, SAMC.

Distribution (Fig. 99). This species is only known from the middle of the winter rainfall belt of the western Cape region of South Africa .

Host plant (77 specimens). Campanulaceae, Wahlenbergia annularis $(32 \% / 45 \hat{0})$.

First - last observations. 02.ix.-23.x.

\section{Capicola rhodostoma Cockerell, 1932}

Capicola rhodostoma Cockerell, 1932b: 175, ․

Hesperapis (Capicola) rhodostoma (Cockerell): Eardley \& Urban, 2006: 164.

Type material. Holotype $q$, South Africa, Foot of Van Rhyn's Pass (?), NHMN. Original designation: "Foot of Van Rhyn's Pass, Cape Province". Cockerell (1932b) described only the female of $C$. rhodostoma. We present here the first description for the male, based on two series of specimens from South Africa, Northern Cape Province Hester Malan Nature Reserve $\left(29.66^{\circ} \mathrm{S}, 17.98^{\circ} \mathrm{E}\right)$ 9.xi.1986 and 7.-27.x.1987, M. Struck, SAMC and Numees, Richtersveld, 4.x.-26.xi.1986, M. Struck, SAMC.

Diagnosis (Figs 71-76). $q$ and $\delta$. Head as wide as long. Labial palpus longer than glossa, as long as maxillary palpus and galea. Outer surface of galea sculptured and mat. Clypeus glabrous, about twice as wide as long, without medio-longitudinal groove. Mesoscutum finely punctate. Propodeal triangle finely rugose, shiny behind; remainder of propodeum shiny. + . $\mathrm{L}=8.4 \mathrm{~mm}$. Integument of lower part of head, head appendages and legs reddish, upper part of head, mesosoma and metasoma black; Compound eyes parallel. Mesoscutum and scutellum with short, appressed, white hairs. T1-T3 black and mat. T1 with continuous white apical fringes, as wide as apical fringes on T2-T4. Spurs of Tb2 with 4-6 large, outstanding spines. Prepygidial fimbria white to very pale brown. Pp with a pair of median, subparallel carinae, areas between and lateral to carinae concave (cf. Figs 9-10). ô (Figs 71-76). L = $6.5 \mathrm{~mm}$. Integument of clypeus, labrum, mandible and antenna reddish to yellow, mesosomal dorsum, dorso-lateral region of propodeum, all legs and T1 reddish-orange. Remainder of integument black or reddish-black. Clypeus, scutum and scutellum shiny, weakly and densely punctate $(\mathrm{i}=\mathrm{d})$. A5 as wide as long. Legs not modified. S6 with short, narrow medioapical spiny processes (Fig. 71). Apex of S7 weakly concave, with small lobes (Fig. 72). S8 with apical plate sub-triangular. S8 and genitalia as illustrated in Figs 73-76.

Description. $\widehat{\delta}$. Head. $\mathrm{L}=2.1 \mathrm{~mm} . \mathrm{W}=2.3 \mathrm{~mm}$. Integument of clypeus, labrum, mandible and antenna reddish to yellowish. Remainder of integument black or reddish-black. Head entirely covered with appressed, white hairs. Labrum swollen. Malar area shorter than A2. 

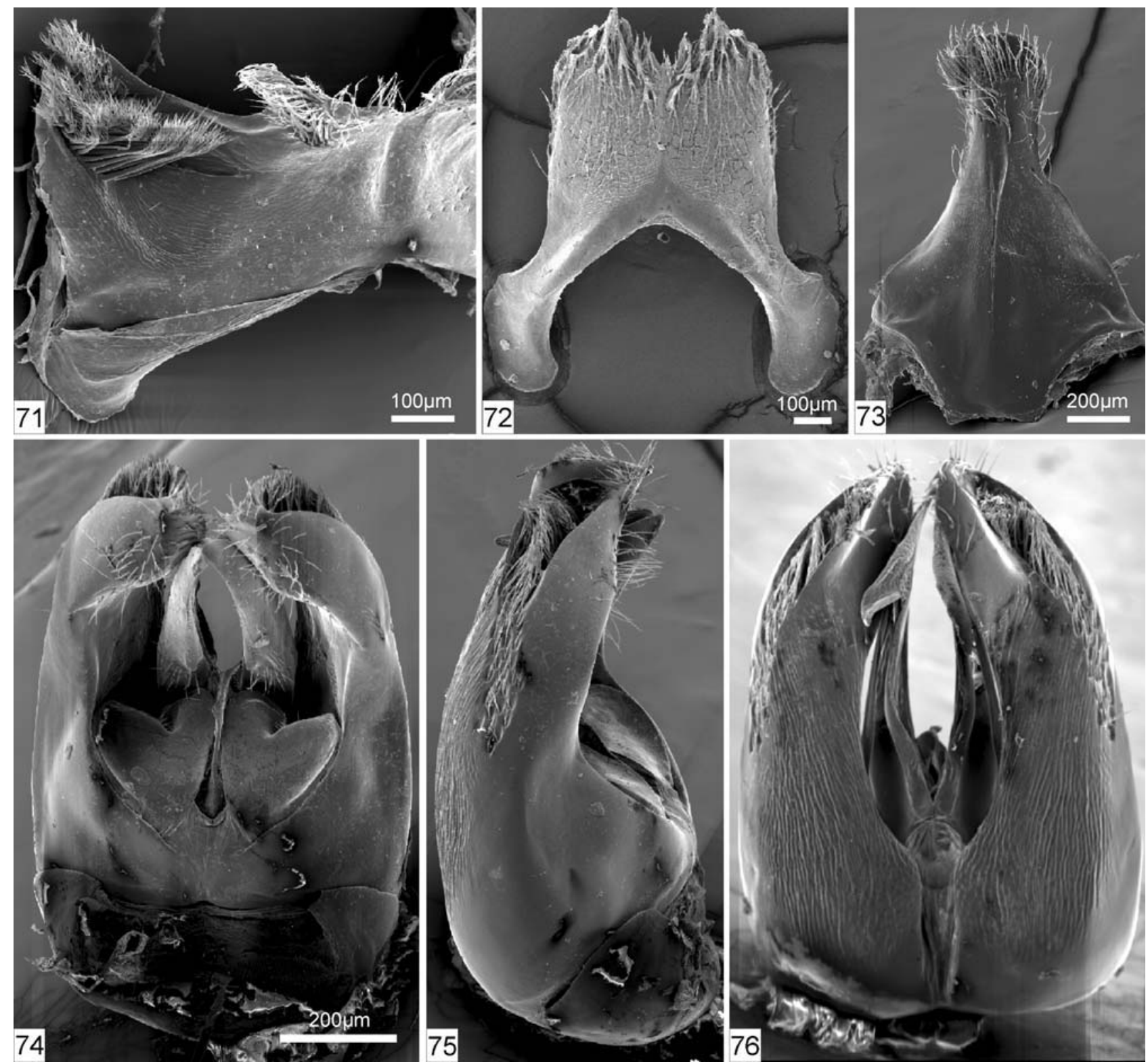

Figs 71-76. Capicola rhodostoma, ô. 71 - lateral view of sternum $6 ; 72$ - ventral view of sternum 7; 73 - ventral view of sternum 8; 74 - ventral view of genitalia; 75 - lateral view of genitalia (scale as in Fig. 74); 76 - dorsal view of genitalia (scale as in Fig. 74).

Clypeus trapezoidal, sparsely covered with appressed, white hairs. Eyes inner margins slightly converging ventrally. Face and vertex with fine, dense, shallow punctures $(i=d)$. Antennae with scape yellowish-black, flagellum yellow. A3 longer than A4. A5 as wide as long. Mesosoma. $\mathrm{L}=2.1 \mathrm{~mm}$. $\mathrm{W}=1.6 \mathrm{~mm}$. Integument of pronotum, scutum, scutellum, metanotum, tegula, axilla and dorsolateral region of propodeum reddish-orange, remainder of mesosoma black. Vestiture pallid, short and appressed on scutum, and long and erect on sides and propodeum length. Propodeal triangle mat anteriorly, glabrous posteriorly. Legs. Integument reddish-black to orange. Mostly with short, brownish, appressed hairs. Legs unmodified. Metasoma. $\mathrm{L}=3.5 \mathrm{~mm}$. $\mathrm{W}=2.3 \mathrm{~mm}$. Integument of $\mathrm{T} 1$ orange, $\mathrm{T} 2-\mathrm{T} 7$ blackish. Vestiture fine and coloured as integument, and terga and sterna finely punctate. S6 with short, narrow medio-apical spiny proc- esses (Fig. 71). Apex of S7 weakly concave, with small lobes (Fig. 72). S8 with apical plate sub-triangular. S8 and genitalia as illustrated in Figs 73-76.

Additional material examined (109 specimens). Namibia: 4오. Churutabis (Bethanie) $\left(27.43^{\circ} \mathrm{S}, 17.47^{\circ} \mathrm{E}\right), 4 .-12 . x .1974$, NMWN; 2 \% , Barby (Bethanie) $\left(25.85^{\circ} \mathrm{S}, 16.55^{\circ} \mathrm{E}\right), \mathrm{NMWN}$; $9+$, Gaiaus (Karasberg) $\left(27.33^{\circ} \mathrm{S}, 18.75^{\circ} \mathrm{E}\right), 13 .-17 . x .1974$, NMWN; 1 을 Aus $\left(26.67^{\circ} \mathrm{S}, 16.27^{\circ} \mathrm{E}\right)$, xii. 1929 , R.E. Turner, AMGS; $1 \delta^{\top}, 17 \mathrm{~km}$ North of Grillenthal, $26.82^{\circ} \mathrm{S}, 15.38^{\circ} \mathrm{E}$, 29.ix.1982, V.B. Whitehead, SAMC. South Africa: $5 \hat{0}$, Garies $\left(30.92^{\circ} \mathrm{S}, 17.97^{\circ} \mathrm{E}\right), 21 . x .1968$, J.G. Rozen, AMNH; 4용ㅎㅇ, Orange River, Vioolsdrif (Namaqualand) $\left(28.77^{\circ} \mathrm{S}, 17.65^{\circ} \mathrm{E}\right)$, 9.x.1988, F.W. \& S.K. Gess, AMGS; 6 $\$ / 1 \delta^{\hat{0}}$, Richtersveld, Koeroegabvlakte, $28.11^{\circ} \mathrm{S}, 17.03^{\circ} \mathrm{E}, 17 .-24 . i x .1995$, F.W. \& S.K. Gess, AMGS; 19 , Namaqualand, Mesklip, $29.48^{\circ} \mathrm{S}$, $17.52^{\circ}$ E, 1.x.1985, F.W. \& S.K. Gess, AMGS; 2 ㅇ, 15 km North Nieuwoudtville $\left(31.38^{\circ} \mathrm{S}, 19.1^{\circ} \mathrm{E}\right)$, on road to Loeriesfontein, 27.ix.1990, F.W. \& S.K. Gess, on Mesembryanthemum sp., 
AMGS; $7 q / 7 \delta$, between Annis and Dabie River, $28.20^{\circ} \mathrm{S}$ $16.55^{\circ}$ E, 19.-20.ix.1997, F.W. \& S.K. Gess, AMGS; 1 ㅇ, Richtersveld, $9 \mathrm{~km}$ ESE Kuboes $\left(28.47^{\circ} \mathrm{S}, 17.43^{\circ} \mathrm{E}\right)$, 25.ix.1995, F.W. \& S.K. Gess, AMGS; 19 , Namaqualand, $66 \mathrm{~km}$ from Vioolsdrif on Eksteenfontein road, $28.50^{\circ} \mathrm{S}, 17.16^{\circ} \mathrm{E}, 6 . x .1994$, F.W. \& S.K. Gess, on white flowers Mesembryanthemum sp., AMGS; 1 \%, Oudtshoorn, Frischgewaagd, $33.39^{\circ} \mathrm{S}, 22.13^{\circ} \mathrm{E}$, 7.-8.xii.1986, S.K. Gess, AMGS; 20 , Namaqualand, Voëlklip, $29.45^{\circ} \mathrm{S}, 17.22^{\circ} \mathrm{E}, 2 .-7 . x .1994$, F.W. \& S.K. Gess, on Wahlenbergia cf. annularis, AMGS; 4\$/60े, Hester Malan Nature Reserve, $29.66^{\circ} \mathrm{S}, 17.98^{\circ} \mathrm{E}, 9 . x i .1986,7 . x .1987,27 . x .1987, \mathrm{M}$. Struck, SAMC; 19 , between Kamieskroon and Springbok, $29.88^{\circ} \mathrm{S}, 17.52^{\circ} \mathrm{E}, \mathrm{x} .1939$, Museum Staff, SAMC; 3 \% , Koup Siding $\left(33.12^{\circ} \mathrm{S}, 21.26^{\circ} \mathrm{E}\right), \mathrm{x} .1952$, Museum Expedition, SAMC; 8 을 Vioolsdrift, Orange River $\left(28.15^{\circ} \mathrm{S}, 17.4^{\circ} \mathrm{E}\right)$ 6.x.1966, SAMC; 5\%, Vioolsdrift, $\left(28.15^{\circ} \mathrm{S}, 17.4^{\circ} \mathrm{E}\right)$, 12.ix.1983, V.B. Whitehead, on Mesembryanthemum sp., SAMC; $10 \% / 30^{\circ}$, Annisfontein, Richtersveld, $28.41^{\circ} \mathrm{S}, 16.88^{\circ} \mathrm{E}$, 23.xi.-23.xii.1975, V.B. Whitehead, SAMC; 2 \% , Richtersveld, Bloeddrif $\left(28.35^{\circ} \mathrm{S}, 16.83^{\circ} \mathrm{E}\right), 20 . x i .1975$, V.B. Whitehead, SAMC; 1 ㅇ, Witputs noord, $\left(31.28^{\circ} \mathrm{S}, 19.40^{\circ} \mathrm{E}\right), 15 . x i .1975$, Whitehead, on Hydrodes sp., SAMC; $19 / 20^{\star}$, Numees, Richtersveld $\left(28.28^{\circ} \mathrm{S}, 16.96^{\circ} \mathrm{E}\right)$, 4.x.-26.xi.1986, M. Struck, SAMC.

Distribution (Fig. 99). Widespread in southern Namibia and the western region of South Africa, mostly in the winter rainfall areas.

Host plant (11 specimens). Aizoaceae, Mesembryanthemum sp. (8 9 ); Campanulaceae, Wahlenbergia cf. annularis (2犬̂), Hydrodes sp. (1 9 )

First - last observations. 17.ix.-8.xii.

\section{Capicola richtersveldensis Patiny \& Michez sp. n.}

Diagnosis (Figs 77-83). $q$ and $\delta$. Integument mostly black, dark brownish on sterna and terga margin. Head wider than long. Labial palpus shorter than glossa and maxillary palpus. Maxillary palpus as long as galea. Outer surface of galea mat and sculptured. Clypeus two and a half times as wide as long, without medio-longitudinal groove, densely punctate $(i=d)$. Propodeal triangle shiny, very short, with declivous basal area. Terga with basal concavity (Fig. 77). ㅇ. L = $10.2 \mathrm{~mm}$. Integument black; sterna margins and Tb3 brownish. Mandibles apex reddish. Flagellum reddish below. Face with white hairs. Head posterior face, mesosoma, legs, T1 and sterna with golden hairs. Spurs of Tb2 with 8-10 sharp outstanding spines. T1-T4 with continuous white, apical fringes. Prepygidial fimbria black to dark brown. Pygidial plate with a strong, high, median carina. ơ (Figs 77-83). L = $10.0 \mathrm{~mm}$. Head wider than long. Eyes not prominent; inner margins converging ventrally. Antennae black. A5 longer than wide. Mesoscutum and scutellum covered with erect, greyish hairs. Tb3 flattened, with anterior region of distal end enlarged (Fig. 80). Bt3 patellate, as wide as long (Fig. 80). Medio-apical processes of S6 with two widely separated, curved processes (Fig. 78). S7 narrowed basally; apex shallowly concave, with two rounded lobes (Fig. 81). S8 with medio-lateral hairy tooth; apex subelliptic (Fig. 79). Genitalia as illustrated in Figs 82-83.

Description. + . Head. $\mathrm{L}=3.0 \mathrm{~mm} ; \mathrm{W}=3.5 \mathrm{~mm}$. Head wider than long. Integument mostly black. Apex of mandibles and clypeus apical lamella reddish. Face covered with white or greyish-white hairs. Vertex with dark brown hairs. Posterior surface covered in golden hairs. Labial palpus shorter than maxillary palpus, reaching apex of galea. Maxillary palpus as long as galea. Outer surface of galea mat. Malar area shorter than A2. Clypeus, face and vertex punctate. Clypeus two and a half times as wide as long, densely punctate $(i=d)$. Compound eyes converging ventrally. Antennae mostly black, except A4-A11 reddish-brown ventrally, A12 entirely reddish. Mesosoma. $\mathrm{L}=4.0 \mathrm{~mm}$. $\mathrm{W}=3.5 \mathrm{~mm}$. Integument black. Mesoscutum and scutellum with a few golden hairs; integument shiny, sparsely punctulate $(i>d)$. Pronotum, pleurae, metanotum and propodeum covered with golden hairs. Propodeal triangle hairless, anterior part mat, posterior part glabrous; short, with declivous basal area. Propodeum shiny. Legs. Integument black, Tb3 a little reddish. Fore and hind legs with golden hairs. Inner surface of $\mathrm{Tb} 2$ with distinct golden hairs. Outer side of mid legs with dense, white appressed hairs. Spurs of Tb2 with eight outstanding spines. $\mathrm{Tb} 3$ and $\mathrm{Bt} 3$ with red spots at base of hairs. Wings. Surface light brownish. Two submarginal cells; first longer than second. Basal vein slightly curved. Metasoma. $\mathrm{L}=6.5 \mathrm{~mm}$. W $=4.5 \mathrm{~mm}$. Terga black. Sterna margin reddish. Terga contiguously punctate, rugose. Discs of terga hairless. T1 with golden hairs. T1-T4 with white, continuous apical fringes. Prepygidial fimbria black on T5, dark brownish on T6. Sterna with very short, pale hairs. S6 with a dense apical fringe of golden hairs. Terga with basal concavity. Sterna and terga with straight premarginal line. Pygidial plate with a strong median carina. $\delta$. Head. $\mathrm{L}=2.7 \mathrm{~mm}$. $\mathrm{W}=$ $3.3 \mathrm{~mm}$. Integument black. Mandible reddish to blackish subapically. Head entirely covered with white, erect hairs. Proboscides as in female. Labrum swollen. Malar area shorter than A2. Clypeus trapezoidal, densely clothed with white hairs. Eyes with inner margins converging ventrally. Face and vertex deeply and densely punctate (i $=\mathrm{d})$. Antennae black. A3 longer than A4. A5 as wide as long. Mesosoma. $\mathrm{L}=3.5 \mathrm{~mm}$. $\mathrm{W}=3.5 \mathrm{~mm}$. Integument black, with long, white, erect hairs; median part of mesoscutum and scutellum a little less hairy (hairs greyish). Nota sparsely punctate. Propodeal triangle as in female. Legs. Integument brown. F1-3 with short, white, erect hairs. External surface of Tb1-3 and tarsi 1-3 with white, erect hairs. Inner surface of tarsi 1-3 with appressed, golden hairs. Tb2 and Bt2 distinctly flattened and enlarged. Tb3 flattened, with apical spine enlarged (Fig. 80). Bt3 patellate (Fig. 80). Metasoma. $\mathrm{L}=6.0 \mathrm{~mm}$. $\mathrm{W}=4.0 \mathrm{~mm}$. Integument black. T1-T4 covered in erect, white hairs. S1-S5 hairless. Disc of terga regularly punctate $(\mathrm{i}>\mathrm{d})$, with basal concavity. Apex of S5 convex. Medio-apical processes of S6 with two widely separated, curved processes (Fig. 78). S7 narrowed basally; apex shallowly concave, with two rounded lobes (Fig. 81). S8 with medio-lateral hairy tooth; apex subelliptic (Fig. 79). Genitalia as illustrated in Figs 82-83.

Type material (25 specimens). Holotype $\hat{\delta} ; 18$ paratypes $\widehat{o}$; 6 paratypes $\%$; South Africa, 6\%/9 $\delta$, near Helskoof gate, $28.18^{\circ} \mathrm{S}, 16.58^{\circ} \mathrm{E}, 12 .-14.1 \times .2001$, on Gorteria, B. Danforth, CUIC; $\widehat{o}$ holotype, 9 đે, idem, C. Eardley, SANC. 


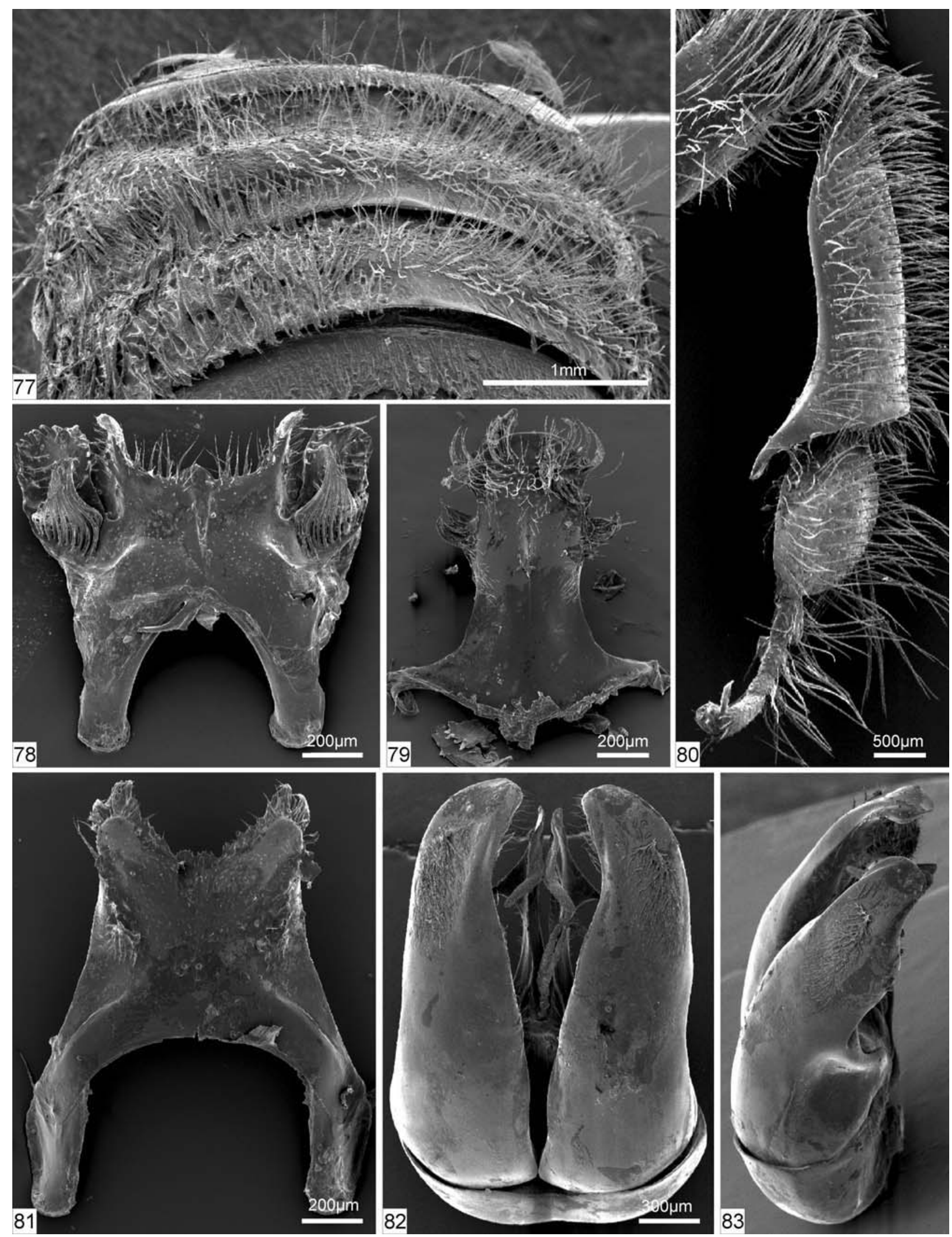

Figs 77-83. Capicola richtersveldensis sp. n., ô. 77 - terga 1-2, showing depressed anterior regions; 78 - ventral view of sternum $6 ; 79$ - ventral view of sternum $8 ; 80$ - outer surface of hind tibia; 81 - ventral view of sternum $7 ; 82$ - dorsal view of genitalia; 83 lateral view of genitalia (scale as in Fig. 82). 
Additional material (28 specimens). 12 $q / 7 \bar{\delta}$, Koeroegabvlakte (Richtersveld), $28.11^{\circ} \mathrm{S}, 17.03^{\circ} \mathrm{E}, 17 .-24.1 x .1995$, F.W. \& S.K. Gess, on yellow flowers of Osteospermum sp., AMGS; 1 ㅇ, Klipfontein (Namaqualand), $29.51^{\circ} \mathrm{S}, 17.47^{\circ} \mathrm{E}$, 14.x.1989, F.W. \& S.K. Gess, on Wahlenbergia cf. annularis, AMGS; $4 \stackrel{+}{9} 7 \mathrm{~km}$ South of Brandvlei $\left(30.5^{\circ} \mathrm{S}, 20.41^{\circ} \mathrm{E}\right)$, 1.xi.1977, V.B. Whitehead, SAMC; 4 ㅇ, Paleisheuwel, $32.28^{\circ} \mathrm{S}$, $18.71^{\circ} \mathrm{E}$, xi.1948, Museum Expedition, SAMC.

Etymology. Named after the type locality.

Distribution (Fig. 96). Only known from the Western and Northern Cape provinces of South Africa.

Host plant (40 specimens). Asteraceae, Gorteria sp. $\left(10 \% / 10 \delta^{\hat{}}\right)$, Osteospermum sp. (12 $\left.\$ / 7 \delta^{\hat{\alpha}}\right)$; Campanulaceae, Wahlenbergia $\mathrm{cf}$. annularis (1 + ).

First - last observations. 12.ix.-14.x.

\section{Capicola rufiventris Friese, 1912}

Capicola (Rhinochaetula) rufiventris Friese, 1912: 186-187, ㅇ․ Capicola (Capicola) rufiventris (Friese): Michener, 1981: 82-83.

Hesperapis (Capicola) rufiventris (Friese): Eardley \& Urban, 2006: 164.

Rhinochaetula femorata Friese, 1925: 504, o.

Capicola (Capicola) femorata (Friese): Michener, 1981: 83.

Capicola callura Cockerell, 1932a: 455, $q$.

Hesperapis callura (Cockerell): Cockerell, 1934: 448.

Capicola (Capicola) callura Cockerell: Michener, 1981: 83.

Capicola nivea Cockerell, 1932a: 456-457, ô.

Hesperapis nivea (Cockerell): Cockerell, 1942: 60.

Capicola (Capicola) nivea Cockerell: Michener, 1981: 83.

Hesperapis faurei Cockerell, 1934: 448. ㅇ.

Type material of $C$. rufiventris (designated here). Lectotype o, South Africa, Ookiep $\left(29.6^{\circ} \mathrm{S}, 17.9^{\circ} \mathrm{E}\right)$, ZMHB. Original designation: "Ookiep, Südafrika". Lectotype: + (ZMBH), labels: $1^{\text {st }}$ printed on white paper "Bushmanld Jackals Water Lightfoot"; $2^{\text {nd }}$ handwritten with black ink on white paper "Rhinochaetula rufiventris 1910 Friese det."; $3^{\text {rd }}$ printed on red paper "Typus"; $4^{\text {th }}$ printed on white paper "Coll. Friese"; $5^{\text {th }}$ printed on red paper "Capicola rufiventris Friese 1912 lectotype design Michez 2006".

Type material of $\boldsymbol{C}$. femorata (designated here). Lectotype $\widehat{ }{ }^{\circ}$, South Africa, Henkries $\left(28.95^{\circ} \mathrm{S}, 18.12^{\circ} \mathrm{E}\right), \mathrm{ZMHB}$. Original designation: "Henkries, Bushmannland". Lectotype: o $(\mathrm{ZMBH})$, labels: $1^{\text {st }}$ handwritten with black ink on white paper "Bushmannld Henkries Fr."; $2^{\text {nd }}$ handwritten with black ink on white paper "Rhinochaetula femorata 1910 Friese det."; $3^{\text {rd }}$ printed on red paper "Typus"; $4^{\text {th }}$ printed on white paper "Coll. Friese"; $5^{\text {th }}$ printed on red paper "Rhinochaetula femorata Friese 1925 lectotype design Michez 2006"; $6^{\text {th }}$ printed on white paper "Capicola rufiventris det. Michez".

Type material of C. callura. Holotype $q$ ?, South Africa, Graaf-Reinet $\left(32.25^{\circ} \mathrm{S}, 24.55^{\circ} \mathrm{E}\right)$, not localised, synonymised by Michener (1981). Original designation: "Graaf-Reinet, Cape Province".

Type material of $\boldsymbol{C}$. nivea. Holotype $\widehat{\delta}$, South Africa, GraafReinet, $\left(32.25^{\circ} \mathrm{S}, 24.55^{\circ} \mathrm{E}\right), \mathrm{NHMN}$. Original designation: "Graaf-Reinet, Cape Province".

Type material of C. faurei. Holotype $q$, South Africa, Brandvlei (?), NHMN. Original designation: "Brandvlei, Cape Province".

Diagnosis (Figs 84-91). 우 and $\delta$. Head wider than long. Labial palpus as long as glossa. Maxillary palpus as long as galea, longer than labial palpus. Outer surface of galea mat. Clypeus twice as wide as long, without median groove. Propodeal triangle with basal area horizontal. $q$ (Fig. 91). $\mathrm{L}=9.6 \mathrm{~mm}$. Clypeus glabrous, with few punctures $(\mathrm{i}>3 \mathrm{~d})$. Mesoscutum and scutellum with small, deep punctures, with appressed hairs. Propodeal triangle with a median groove; anterior half finely rugose to strigulate; posterior half shiny and unsculptured. Posterior part of propodeum shiny. Fore and mid legs with whitish hairs. Spurs of Tb2 with 4-6 sharp outstanding spines. Terga mat, weakly punctate. T1 with interrupted apical hair band (some specimens with reduced but continuous band). T1-T3 orange. T3 with lateral brown spot. Prepygidial fimbria white brownish to brownish. Pp with a strong elevated median area; not tapering (Fig. 91). $\delta$ (Figs 84-90). $\mathrm{L}=7.8 \mathrm{~mm}$. Clypeus densely punctate $(\mathrm{i}=$ d), covered with long white appressed hairs. A4-13 dorsally brown, ventrally reddish. A3 as long as A4. A5 as long as wide. Mesoscutum and scutellum shiny between small, weak punctures. Legs with white appressed hairs. Tb3 apex widely expanded. Medio-apical process of S6 blade shaped (Figs 84, 87). S7 with spiny apico-lateral processes (Figs 85, 88). Apex of S8 subelliptic. S8 and genitalia as illustrated in Figs 86, 89-91.

Additional material examined (196 specimens). Namibia: 10 \% $/ 240^{\Uparrow}$, Mariental $\left(24.63^{\circ} \mathrm{S}, 17.97^{\circ} \mathrm{E}\right), 24 . x .1968$, J.G. Rozen, AMNH; $15 \% / 10^{\star}$, Barby (Bethanie) $\left(25.85^{\circ} \mathrm{S}, 16.55^{\circ} \mathrm{E}\right)$, NMWN; 19 , Aurusberge $\left(27.65^{\circ} \mathrm{S}, 16.31^{\circ} \mathrm{E}\right), 22 . x .1974$, R. Watmough, SANC; 10 , Skeleton Coast Park, Geanias Water Hole $\left(19.12^{\circ} \mathrm{S}, 12.87^{\circ} \mathrm{E}\right), 24.1 .1983$, V.B. Whitehead, SAMC; 2 ㅇ, Klinghardt Mountains $\left(27.37^{\circ} \mathrm{S}, 15.87^{\circ} \mathrm{E}\right)$, x.1982, V.B.

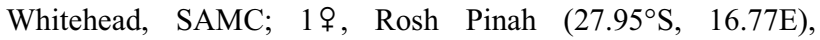
18.xi.1975, V.B. Whitehead, SAMC. South Africa: $10^{\hat{}}$, Graaf Reinet $\left(32.25^{\circ} \mathrm{S}, 24.55^{\circ} \mathrm{E}\right)$, 24.-27.x.1931, BMNH; 1 잉ㅎㅇ, Cradock $\left(32.18^{\circ} \mathrm{S}, 25.62^{\circ} \mathrm{E}\right), 30 . x i .1966$, J.G. Rozen, AMNH; 1 ㅇ, Keetmanshoop $\left(26.58^{\circ} \mathrm{S}, 18.13^{\circ} \mathrm{E}\right)$, 23.x.1968, J.G. Rozen, AMNH; 2 + , Vioolsdrif $\left(28.77^{\circ} \mathrm{S}, 17.65^{\circ} \mathrm{E}\right), 19 . x .1999$, Halada, OOLL; $22 \% / 280^{\star}$, Murraysburg, $31.95^{\circ} \mathrm{S}, 23.76^{\circ} \mathrm{E}$, xi. 1935 , Museum Staff, SAMC; $43 \%$, Upington, $28.45^{\circ} \mathrm{S}, 21.25^{\circ} \mathrm{E}$, 10.-12.x.1966, SAMC; 2 $/ 1 \mathrm{o}^{\hat{}}$, Williston, $31.35^{\circ} \mathrm{S}, 20.92^{\circ} \mathrm{E}$, 30.xi.1978, V.B. Whitehead, on Psilocaulon sp., SAMC; 19 , idem, on Aloe sp.; $19 / 1$ ô, idem, on Mesembryanthemum crystalinum; 10 요의, Fraserburg, Klipheuwels, $31.92^{\circ} \mathrm{S}, 21.48^{\circ} \mathrm{E}$, 29.xi.1978, V.B. Whitehead, on Psilocaulon sp., SAMC; 1 , Witputs $\left(27.27^{\circ} \mathrm{S}, 20.35^{\circ} \mathrm{E}\right)$, 15.x.1975, V.B. Whitehead, on Mesembryanthemum sp., SAMC; 5 , Loxton District, Dunedin farm, $31.47^{\circ} \mathrm{S}, 22.42^{\circ} \mathrm{E}, 29 . x i .1974$, V.B. Whitehead, SAMC; 3 ㅇ, Putsonderwater, $29.23^{\circ} \mathrm{S}, 21.88^{\circ} \mathrm{E}, \mathrm{x} .1939$, Museum Staff, SAMC; 1 ㅇ, Richmond District $\left(31.42^{\circ} \mathrm{S}, 23.93^{\circ} \mathrm{E}\right)$, xi.1939, Museum Staff, SAMC; 10, Naib, Bushmanland, between Springbok and Pella, $29.35^{\circ} \mathrm{S}, 18.35^{\circ} \mathrm{E}$, x.1939, Museum Staff, SAMC; 2 ㅇ, Annisfontein, Richtersveld, $28.38^{\circ} \mathrm{S}, 15.87^{\circ} \mathrm{E}$, 20.-23.xi.1975, V.B. Whitehead, SAMC; 6요 Leliefontein, Paulshoek, $30.38^{\circ} \mathrm{S}, 18.27^{\circ} \mathrm{E}, 28 .-30 . i x .1997$, C. Seymour, SAMC.

Distribution (Fig. 97). Widespread through Namibia and the western part of South Africa.

Host plant (24 specimens). Aizoaceae, Aloe sp. (1 9 ), Psilocaulon sp. (12\%/9ð), Mesembryanthemum sp. (1\%) and Mesembryanthemum crystalinum (1 9 ).

First - last observations. 10.x. -30 .xi.

\section{KEY TO SPECIES}

1 Head longer than wide. Scutum and scutellum orangish. Pp $\uparrow$ without medio-longitudinal carinae... C. flavicara sp. n. 

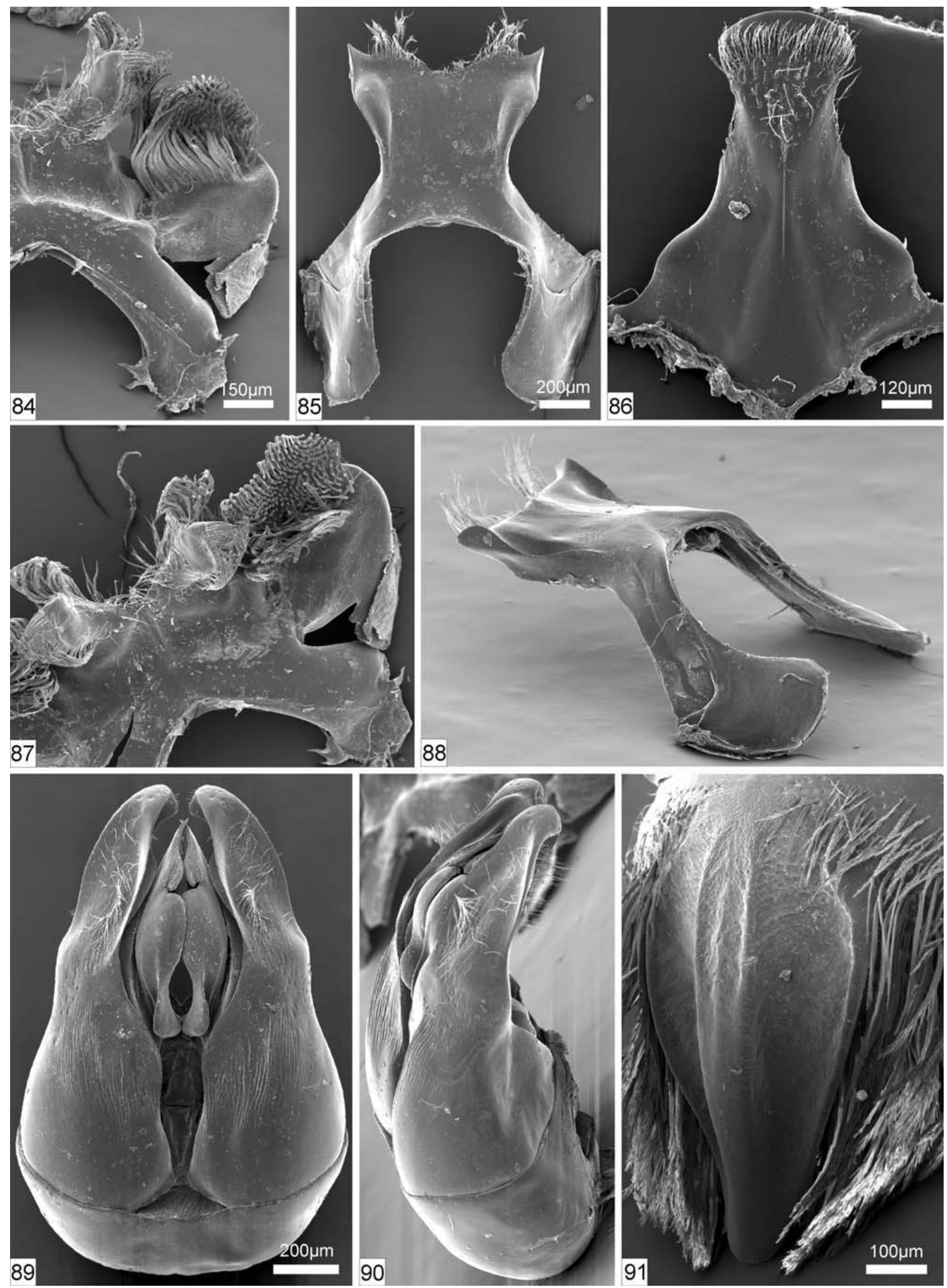

Figs 84-91. Capicola rufiventris. 84 - ventral view of sternum $6 \hat{\delta} ; 85$ - ventral view of sternum $7 \hat{\delta} ; 86$ - ventral view of sternum $8 \hat{\jmath} ; 87$ - posterior view of sternum $6 \hat{0}$ (scale as in Fig. 84); 88 - lateral view of sternum $7 \hat{\jmath}$ (scale as in Fig. 85); 89 - dorsal view of genitalia $\delta^{\lambda} ; 90$ - lateral view of genitalia $\widehat{\delta}$ (scale as in Fig. 89). 91 - lateral view of pygidial plate $\$$. 

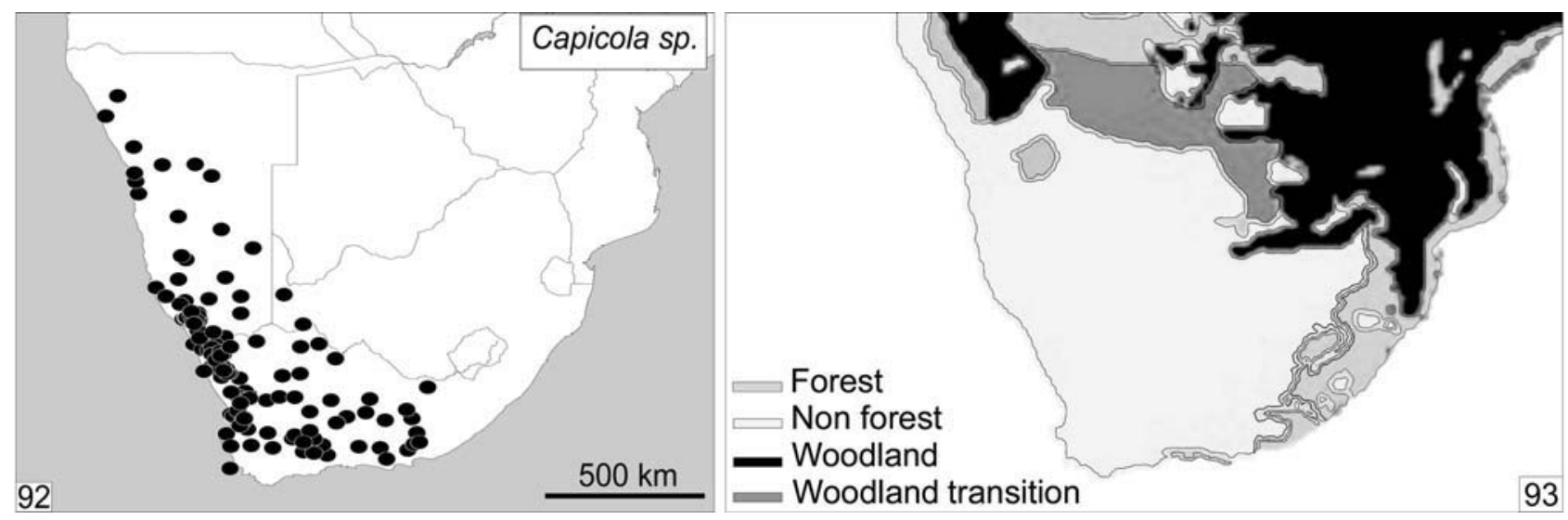

Figs 92-93. 92 - collecting localities of the genus Capicola (1136 specimens); 93 - general distribution of ecosystems in Southern Africa (White, 1986).

- Head as long as wide or wider than long. Scutum and scutellum mostly black. Pp $\uparrow$ with medio-longitudinal carinae. .

2 Outer surface of $\mathrm{Tb} 3+$ with large, blunt, spine-like hairs (Fig. 46). Prepygidial fimbria $q$ brown. Disc of S6 without medio-longitudinal groove. Disc of $\mathrm{S} 7 \hat{\sigma}$ narrowed medially; apical lobes diverging (Figs 5, 53)......... 3

- Outer surface of Tb3 $q$ without large, blunt, spine-like hairs. Prepygidial fimbria + orange to black. Disc of S6 $\delta$ with medio-longitudinal groove (Figs 20-21). Disc of S7 $\delta$ not narrowed; apex generally emarginate, lobes not

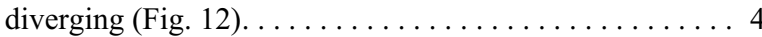

3 Clypeus twice as wide as long. Hind part of propodeal triangle impunctate, smooth and shiny. Body length $>6.5 \mathrm{~mm}$. Metasoma + black. S2-S4 $\delta$ with dense white apical hair bands. Apical area of S8 $\delta$ flattened. S7-S8 ô and genitalia as figured in Figs $5-8 \ldots \ldots \ldots \ldots \ldots \ldots$. aliciae

- Clypeus three times as wide as long. Propodeal triangle entirely rugose (Fig. 44). Body length $<6.5 \mathrm{~mm}$. Metasoma o mainly reddish. S2-S4 $\delta$ with few apical hairs. Apical area of S8 ot circular (Fig. 54). S7-8 ot and genitalia as figured in Figs $52-57 \ldots \ldots \ldots \ldots \ldots$. micheneri sp. n.

4 Females (Figs 1-2). Antenna 12 segmented. Tb3 and Bt3 with scopa. . . . . . . . . . . . . . . . . 5

- Males (Figs 3-4). Antenna 13 segmented. Tb3 and Bt3

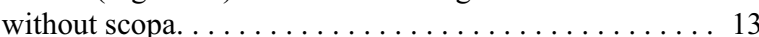

5 Clypeus three times as wide as long ( $3: 1)$. T3-T6 orangish. $\mathrm{Pp}$ as figured in Figs $58-59 \ldots \ldots \ldots \ldots \ldots$. . nanula

- Clypeus relatively longer, two and a half times to twice as wide as long (2.5: 1 to $2: 1)$. T3-T6 mostly black. Pp different. . . . . . . . . . . . . . . . . . . . 6

6 Clypeus twice as wide as long $(2: 1)$, with few superficial punctures $(\mathrm{i}>3 \mathrm{~d}) \ldots \ldots \ldots \ldots \ldots \ldots \ldots \ldots \ldots$

- Clypeus two and an half times as wide as long (2.5:1), with dense, deep punctures $(\mathrm{i}<\mathrm{d})$ at least basally. . . . . . . . 9

7 T1-T3 orange. T1 with medially interrupted apical fringe, smaller than apical fringes of T2-T4. Pp with a strongly elevated median area (Fig. 91). Head wider than long. Compound eyes parallel.

C. rufiventris

- T1-T3 black to reddish. T1 with continuous apical fringe, as dense as apical fringes of T2-T4. Pp with a weak median elevated area consisting of a pair of median subparallel carinae, area between these carinae concave (Figs 9-10). Head as wide as long. Compound eyes converging below..... 8

8 Clypeus and supraclypeal area reddish. Propodeal triangle with declivous basal area............. C. rhodostoma
- Clypeus mostly black, sometimes apical area reddish. Supraclypeal area black. Propodeal triangle with horizontal basal area. . . . . . . . . . . . . . . . . . . . . C. braunsiana

9 Prepygidial fimbria yellow or orange. . . . . . . . 10

- Prepygidial fimbria brown or black. . . . . . . . . . . . 12

10 Body length $<7.0 \mathrm{~mm}$. Integument of $\mathrm{T} 1-4$ reddish. . . . . . $\ldots \ldots \ldots \ldots \ldots \ldots \ldots \ldots \ldots$. gessorum sp. $n$.

— Body length $>7.0 \mathrm{~mm}$. Integument of T1-4 black. . . . . 11

11 Scutum with shallow punctures. Outer surface of galea sculptured. Disc of T2-T5 shiny, without basal concavity. Spurs of Tb2 with 4-6 large spines. Body length $<8.0 \mathrm{~mm}$. . C. flavitarsis

- Scutum with deep punctures. Outer surface of galea smooth. Disc of terga mat, with basal concavity. Spurs of Tb2 without large spine. Body length $>10.0 \mathrm{~mm} \ldots \ldots \ldots \ldots$ ....................... danforthi sp. n.

12 Disc of terga with shallow punctures. Outer surface of galea sculptured.............. C. richtersveldensis $\mathrm{sp.} \mathrm{n}$.

- Disc of terga with deep punctures. Outer surface of galea smooth. . . . . . ............... . nigerrima

13 A4-10 yellow, A11-13 dark. Clypeus three times as wide as long $(3: 1) \ldots \ldots \ldots \ldots \ldots \ldots \ldots \ldots \ldots \ldots \ldots \ldots \ldots$. nanula

- All antennal segments with the same colour. Clypeus relatively longer, two and a half times to twice as wide as long

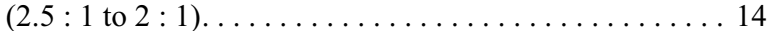

$14 \mathrm{~Tb} 3$ not expanded apically. Gonostylus with narrow hairy groove (margin of groove parallel) (Figs 15-16, 76). S6 with medio-apical small teeth (Figs 11,71). . . . . . 15

- Tb3 expanded apically. Gonostylus with large hairy groove (margin of groove not parallel) or hairless groove. S6 with medio-apical curved blade (Figs $20-21$ ) . . . . . . . . . 16

15 Clypeus with dense punctures $(i<d)$ at least basally. Labrum, clypeus and supraclypeal area reddish. Compound eyes converging below. ........... C. rhodostoma

- Clypeus with few punctures $(\mathrm{i}>3 \mathrm{~d})$. Clypeus mostly black, sometimes apical area reddish. Supraclypeal area black. Compound eyes sub-parallel. ........... C. braunsiana

16 Clypeus twice as wide as long $(2: 1)$. Hind leg orange. S7 with latero-apical teeth (Figs 85, 88). S6, S8 and genitalia as figured in Figs 84, 86-87, 89-90........ C. rufiventris

- Clypeus two and a half times as wide as long $(2.5: 1)$. Hind leg black to brownish. S6-S8 and genitalia different. . . . 17

17 Disc of T2-4 flat. Eyes prominent (Fig. 34). Medio-apical process of S6 spiny shaped (Fig. 32). S7 and genitalia as in Figs 33, 35-37. ................ flavitarsis

- Disc of T2-4 with basal concavity. Eyes not prominent. S7 and genitalia different. . . . . . . . . . . . . 18 

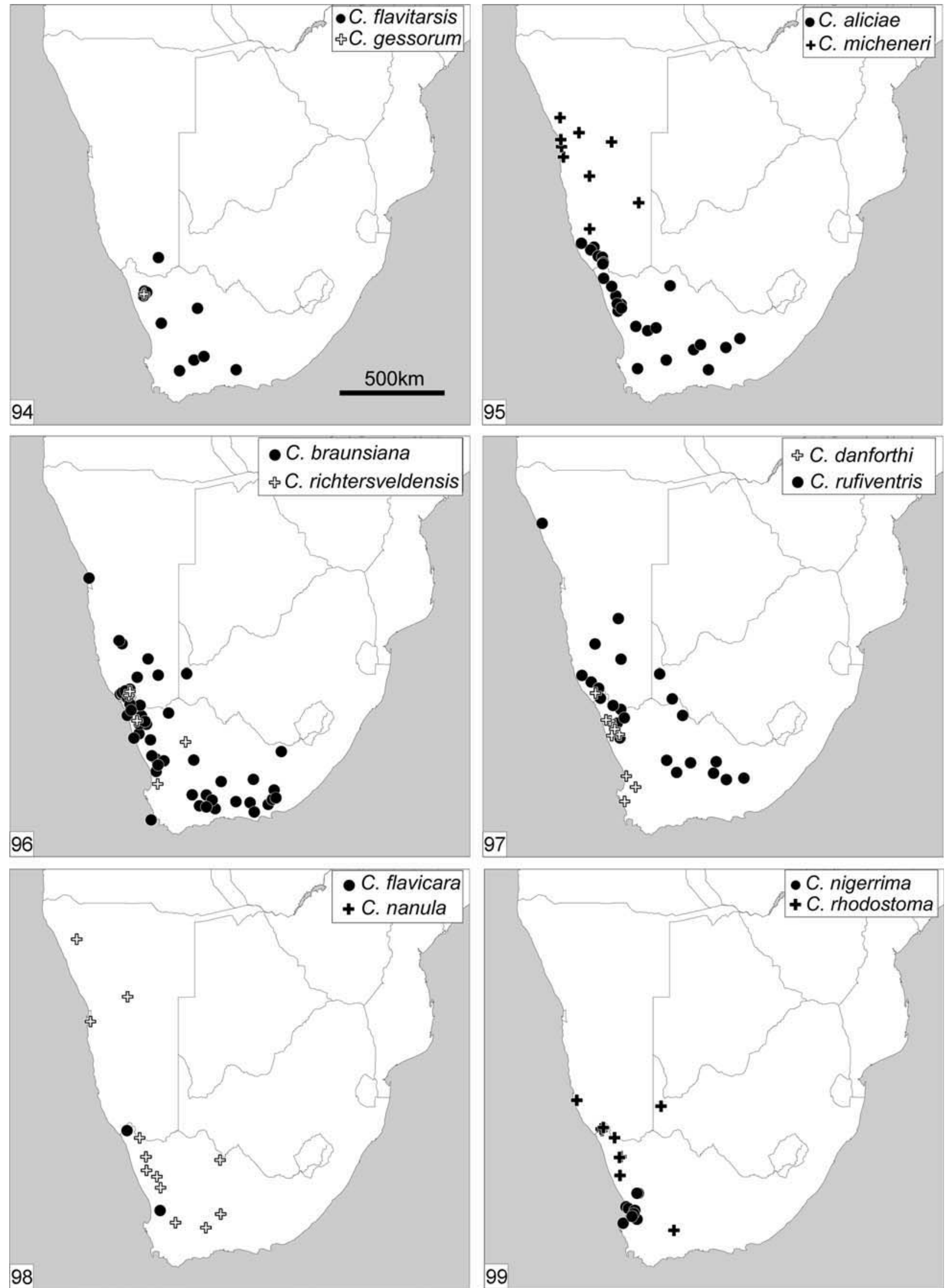

Figs 94-99. Collecting localities of Capicola species. 94 - C. flavitarsis (circles, 21 specimens) and C. gessorum sp. n. (crosse, 16 specimens); 95 - C. aliciae (circles, 108 specimens) and C. micheneri sp. n. (crosses, 75 specimens); 96 - C. braunsiana (circles, 292 specimens) and $C$. richtersveldensis sp. n. (crosses, 59 specimens); $97-C$. danforthi sp. n. (crosses, 211 specimens) and $C$. rufiventris (circles, 196 specimens); $98-$ C. flavicara sp. n. (circles, 4 specimens) and C. nanula (crosses, 94 specimens). 99 , $C$. nigerrima (crosses, 107 specimens) and C. rhodostoma (circles, 109 specimens). 


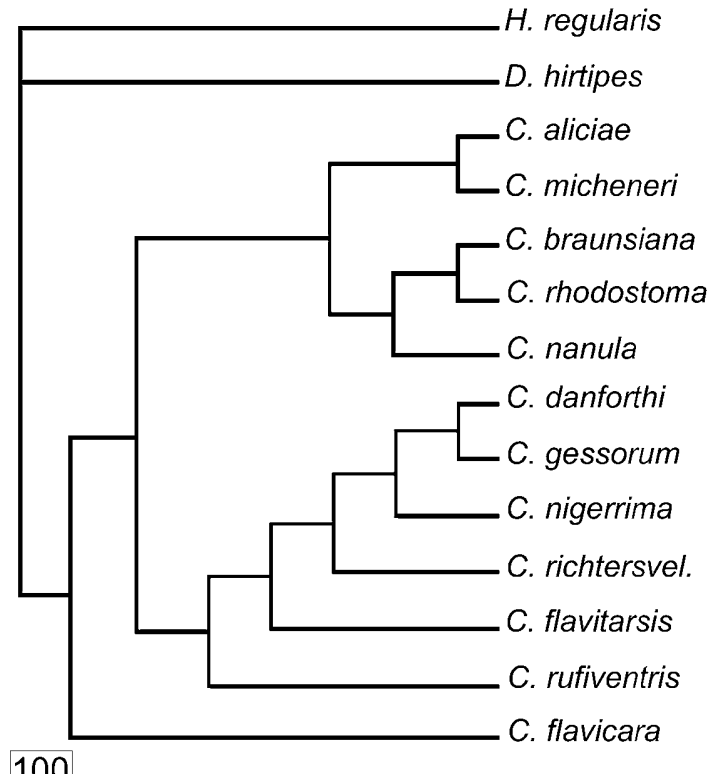

100

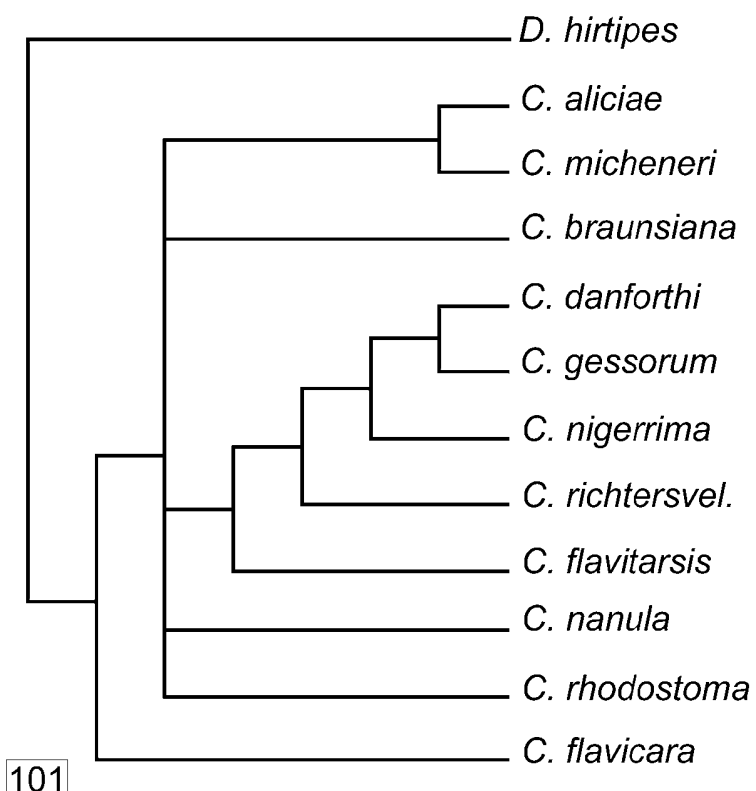

101

Figs 100-101. 100 - strict consensus of most parsimonious trees (length $=46, \mathrm{CI}=0.6087$ and $\mathrm{RI}=0.7465)$ from heuristic search; $101-$ strict consensus of most parsimonious trees (length $=44, \mathrm{CI}=0.6364$ and $\mathrm{RI}=0.7288$ ) from exhaustive search.

18 Body length $<5.0 \mathrm{~mm}$. Tb3 apically weakly toothed (Fig. 43), with long white hairs. S6-S8 and genitalia as in figured in Figs $38-42 \ldots \ldots \ldots \ldots \ldots \ldots$. . gessorum sp. $n$.

- Body length $>5.0 \mathrm{~mm}$. Tb3 apically broadened (Figs 19, 64, 80). S6-S8 and genitalia different. ............ 19

19 Bt3 patellate, as wide as long (Fig. 80). Apex of S7 with two differentiated rounded lobes (Fig. 81). S8 with medio-lateral hairy tooth (Fig. 79). S6 and genitalia as in Figs 78, 82-83. . $\ldots \ldots \ldots \ldots \ldots \ldots \ldots$. richtersveldensis sp. $\mathrm{n}$.

- Bt3 normal, three times as long as wide. Apex of S7 with small lobes (Fig 22). S8 without hairy tooth (Fig. 65). S6 and genitalia different. . . . . . . . . . . . . . 20

$20 \mathrm{~Tb} 3$ with a tooth between the apical spurs (Fig. 19). Clypeus without medio-longitudinal groove. Disc of terga moderately densely punctate. S6 with medio-apical processes blade shaped (Figs 20-21). Genitalia as in Figs 23-25. . . . . ......................... danforthi sp. n.

- Tb3 without a tooth between the apical spurs. Clypeus with a medio-longitudinal groove. Disc of terga with dense punctation. S6 with medio-apical processes swollen (Fig. 66). Genitalia as in Figs $68-70 \ldots \ldots \ldots \ldots$. . nigerrima

\section{DISCUSSION}

\section{Morphology and taxonomy}

The species included in Capicola are unambiguously characterized by some morphological structures like proboscis proportions, body size, punctuation of clypeus, propodeal triangle, shape of hidden sterna ôे (S6-S8) and genitalia $\hat{\sigma}$. The shape of the females' pygidial plate is diagnostic for many species, what is notably unusual in melittids (except in Dasypoda females; Michez et al., 2004a).

Our cladistic analysis does not support the former subdivision of Capicola into two subgenera Capicoloides and Capicola (Michener, 1981, 2000). The group described by Michener (1981) as Capicoloides is monophyletic, robust and supported by high bootstrap values (Fig. 100). However, the large species group designated as Capicola s.str. is obviously not monophyletic. The five species originally included in the latter taxon $(C$. braunsiana, C. cinctiventris (=C. flavitarsis), C. flavitarsis, $C$. nanula, $C$. rufiventris) are associated in two distinct clades. Moreover, the relationships of three species $(C$. braunsiana, C. nanula and C. rhodostoma) remain uncertain based on the available knowledge. Acknowledging subgenera in Capicola based on the previous trees would consequently lead to numerous taxonomical changes, a critical taxonomic inflation and no significant improvement of the systematic relevance. We propose thus to pool the twelve species in one single genus including several (at least three) monophyletic groups of species.

At a higher taxonomic level, our previous proposal leads to the acknowledgement of seven subgenera - all Nearctic - within Hesperapis namely: Amblyapis Cockerell, 1910, Carinapis Stage, 1981, Disparapis Stage, 1981, Hesperapis s. str., Panurgomia Viereck, 1909, Xeralictoides Stage, 1981 and Zacesta Ashmead, 1899. Moreover, the Dasypodaini would then include four genera: Capicola, Dasypoda Latreille, 1802, Eremaphanta and Hesperapis. The phylogenetic relationships among these genera need re-evaluation through a global analysis. Interesting preliminary clues on the result of such analysis were suggested by Engel (2005) who grouped Capicola and Eremaphanta within the subtribe Hesperapina. Several of our own observations give us additional insights supporting this proposal, notably in the $C$. flavicara morphology (flatness of the female's pygidial plate and the shape of the head).

Other questions concerning the higher taxonomic levels are (1) the taxonomic status of sub-genera of Hesperapis; (2) the nature of the phylogenetic relationships between these taxa, and (3) the relationships between Dasypodaini and the other tribes of the Dasypodainae (Promelittini and Sambini). 


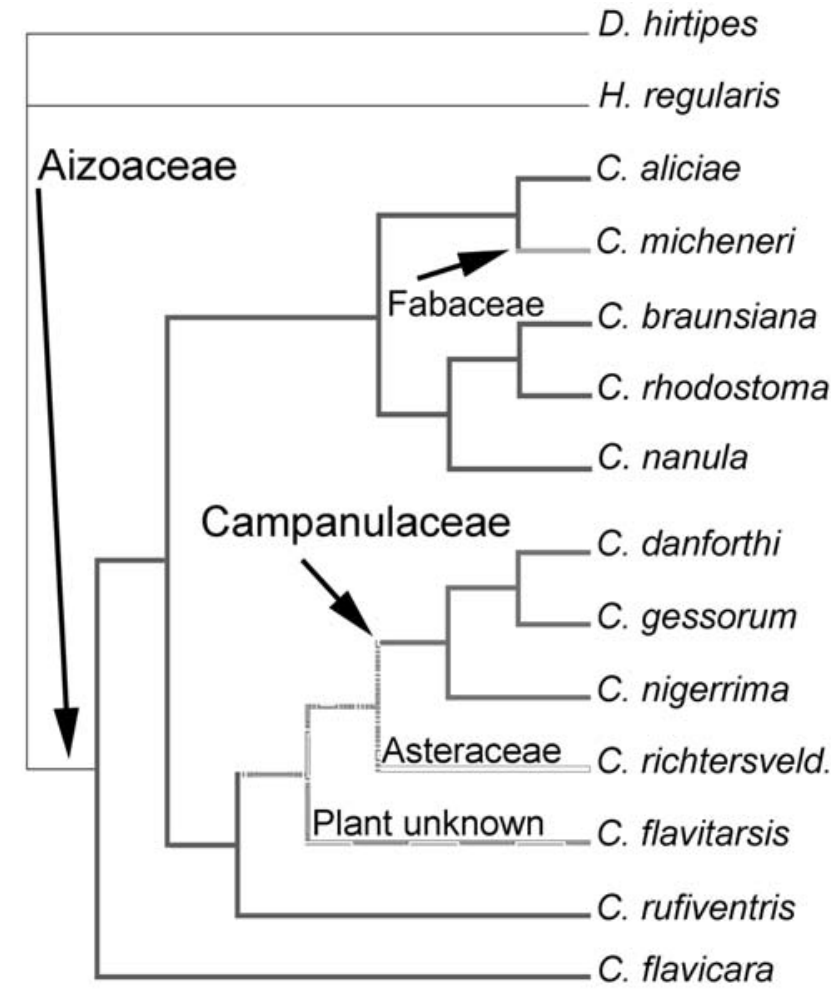

Fig. 102. Phylogeny and host plants of Capicola.

\section{Biogeography}

Capicola distribution is mostly restricted to the xeric part of south-western Africa (Figs 92-93). The diversity pattern seems unipolar (Figs 94-99) contrary to the bipolar one of species like those in Colletes Latreille, 1802 (Kuhlmann, 2005). The centre of endemism is clearly in the Western Cape Province's Succulent and Nama Karoo biomes, which have the world's highest temperate floral diversities (Kuhlmann, 2005). The distributions of several species extend northward into Namibia and eastward in the Eastern Cape Province. One species that is recorded outside this area is $C$. micheneri which is an Namibian endemic species.
The narrow range of Capicola is remarkable compared to the distribution of their closest relatives among Dasypodaini: Hesperapis and Eremaphanta, which are respectively Nearctic and Central Asian (Michener, 1981; Michez \& Patiny, 2006). The distribution of the group formed by these three taxa includes two large disjunctions, the origins of which could be well explained by a serie of extinction events. The potential causes for these extinctions are likely to be the consequences of major climate changes undergone in these regions during the Quaternary period (Marchant \& Hooghiemstra, 2004).

\section{Host plants}

Capicola species seem to be oligolectic on a few plant families: Aizoaceae (6 species), Asteraceae (1 species), Campanulaceae (3 species) and Fabaceae (1 species) (Table 2). These data confirm the analysis of Gess \& Gess (2004).

The likely host plant specialisation of Capicola confirms the oligolectic feature of Melittidae. In fact, most other melittid genera are described as oligolectic: Macropis on Lysimachia sp., Primulaceae (Michez \& Patiny, 2005); some Eremaphanta on Convolvulus sp., Convolvulaceae (Popov, 1940; Michez \& Patiny, 2006); the subgenus Dasypoda s. str. on Asteraceae (Michez et al., 2004a, b).

The host plants of Capicola are very common in South Africa. The families Aizoaceae, Asteraceae and Fabaceae are also very attractive to many other oligolectic bee species (Gess \& Gess, 2004).

\section{Host plant in the context of Capicola phylogeny}

The results of the phylogenetic analysis seem coherent with the host plants (Fig. 102). Most of the robust monophyletic groups seem to be specialized on only one plant family. The group of $C$. flavicara and $C$. aliciae seem to be oligolectic on Aizoaceae and $C$. micheneri has switched to Fabaceae. In the group of $C$. danforthi, $C$. gessorum and $C$. nigerrima the host plants are more diverse (Aizoaceae, Asteraceae and Campanulaceae). $C$. rufiventris is potentially oligolectic on Aizoaceae, and it is the sister species of the former group. It is possible that Aizoaceae is the "plesiomorphic" floral choice for Capi-

TABLE 2. Flower visits (in percentage) of Capicola species (number of specimens in brackets).

\begin{tabular}{|c|c|c|c|c|c|}
\hline \multirow{2}{*}{ Capicola species } & \multicolumn{5}{|c|}{ Host plant } \\
\hline & Aizoaceae & Asteraceae & Campanulaceae & Fabaceae & Molluginaceae \\
\hline C. aliciae (6) & 100 & - & - & - & - \\
\hline C. braunsiana (32) & 100 & - & - & - & - \\
\hline C. danforthi (79) & - & - & 100 & - & - \\
\hline C. flavicara (1) & 100 & - & - & - & - \\
\hline C. gessorum (13) & - & - & 100 & - & - \\
\hline C. micheneri (66) & - & - & - & 97 & 3 \\
\hline C. nanula (21) & 95 & - & 5 & - & - \\
\hline C. nigerrima (77) & - & - & 100 & - & - \\
\hline C. rhodostoma (11) & 73 & - & 27 & - & - \\
\hline C. richtersveldensis (40) & - & 98 & 2 & - & - \\
\hline C. rufiventris (24) & 100 & - & - & - & - \\
\hline
\end{tabular}


cola. According to the tree topology, the specialisation on Fabaceae, Asteraceae and Campanulaceae has each evolved once.

ACKNOWLEDGEMENTS. We sincerely thank the curators of the studied collections: J. Ascher (AMNH), M. Cochrane (SAMC), F. \& S. Gess (AMGS), F. Gusenleitner (OOLL), E. Marais (NMWN), J.G. Rozen (AMNH). Thanks also to P. Rasmont (Mons, Belgium), M. Terzo (Mons, Belgium) and N.J. Vereecken (Brussel, Belgium) for their kind proof reading. S. Patiny is a FNRS (Fond National de la Recherche Scientifique) Postdoctoral researcher.

\section{REFERENCES}

Barbier Y. \& Rasmont P. 2000: Carto Fauna-Flora 2.0. Guide d'utilisation. Université de Mons-Hainaut, Mons, 59 pp.

Barbier Y., Rasmont P., Dufrêne M. \& Sibert J.M. 2000: Data Fauna-Flora. Guide d'utilisation. Université de MonsHainaut, Mons, $107 \mathrm{pp}$.

Cockerell T.D.A. 1915: Descriptions and records of bees. LXVI. Ann. Mag. Nat. Hist. 8: 341-350.

Cockerell T.D.A. 1932a: Descriptions and records of bees. CXXXII. Ann. Mag. Nat. Hist. 10: 447-458.

Cockerell T.D.A. 1932b: Descriptions and records of bees. CXXXV. Ann. Mag. Nat. Hist. 10: 166-176.

Cockerell T.D.A. 1932c: Discontinuous distribution in bees. Nature 130: 58-59.

Cockerell T.D.A. 1934: Descriptions and records of bees. CXLVIII. Ann. Mag. Nat. Hist. 10: 444-456.

Cockerell T.D.A. 1936a: Descriptions and records of bees. CLII. Ann. Mag. Nat. Hist. 10: 24-31.

Cockerell T.D.A. 1936b: Descriptions and record of bees. CLIV. Ann. Mag. Nat. Hist. 10: 477-483.

Cockerell T.D.A. 1942: Descriptions and records of bees. CLXXXIV. Ann. Mag. Nat. Hist. 11: 56-60.

EARDLEY C.D. \& URban R.P. 2006: Taxonomic name changes in Afrotropical bees (Hymenoptera: Apoidea). Afr. Entomol. 14: 161-174.

ENGEL M.S. 2001: A monograph of the Baltic Amber bees and evolution of the Apoidea (Hymenoptera). Bull. Am. Mus. Nat. Hist. 259, 192 pp.

ENGEL M.S. 2005: Family-group names for bees (Hymenoptera: Apoidea). Am. Mus. Nov. 3476: 1-33.

FrIESE H. 1911: Neue Bienen der äthiopischen Region. Zool. Jb. Abt. Syst. Geogr. Biol. Tiere 30: 671-678.

FrIESE H. 1912: Neue und wenig bekannte Bienen Süd-Afrikas (Hym.). Arch. Naturgesch. 78: 181-189.

Friese H. 1925: IV. Nachtrag zu "Bienen Afrikas". Zool. Jb. Abt. Syst. Geogr. Biol. Tiere 49: 501-512.
Gess S.K. \& Gess F.W. 2004: A comparative overview of flower visiting by non-Apis bees in the semi-arid to arid areas of southern Africa. J. Kans. Entomol. Soc. 77: 602-618.

HaRris R.A. 1979: A glossary of surface sculpturing. Occas. Pap. Entomol. 28: 1-31.

Kunlmann M. 2005: Diversity, distribution patterns and endemism of southern African bees (Hymenoptera: Apoidea). In Huber B.A., Sinclair B.J. \& Lampe K.-H.: African Biodiversity: Molecules, Organisms, Ecosystems. Springer Verlag, Bonn, pp. 167-172.

Marchant R. \& Hooghiemstra H. 2004: Rapid environmental change in African and South American tropics around 4000 years before present: a review. Earth-Sci. Rev. 66: 217-260.

Michener C.D. 1981: Classification of the bee family Melittidae with a review of species of Meganomiinae. Contr. Am. Entomol. Inst. 18, $135 \mathrm{pp}$.

Michener C.D. 2000: The Bees of the World. The Johns Hopkins University Press, Baltimore, $913 \mathrm{pp}$.

Michez D. \& Patiny S. 2005: World revision of the oilcollecting bee genus Macropis Panzer 1809 (Hymenoptera, Apoidea, Melittidae) with a description of a new species from Laos. Ann. Soc. Entomol. Fr. (N.S.) 41: 15-28.

Michez D. \& Patiny S. 2006: Review of the bee genus Eremaphanta Popov 1940 (Hymenoptera: Melittidae), with the description of a new species. Zootaxa 1148: 47-68.

Michez D., Terzo M. \& Rasmont P. 2004a: Révision des espèces ouest-paléarctiques du genre Dasypoda Latreille 1802 (Hymenoptera, Apoidea, Melittidae). Linz. Biol. Beitr. 36: 847-900.

Michez D., Terzo M. \& Rasmont P. 2004b: Phylogénie, biogéographie et choix floraux des abeilles oligolectiques du genre Dasypoda Latreille 1802 (Hymenoptera, Apoidea, Melittidae). Ann. Soc. Entomol. Fr. (N.S.) 40: 421-435.

Popov V.V. 1940: A new genus of bees from Turkestan (Hymenoptera, Panurgidae). Trav. Inst. Zool. Acad. Sci. U.R.S.S. 6: 53-59.

Rozen J.G. 1974: The biology of two African melittid bees (Hymenoptera, Apoidea). N.Y. Entomol. Soc. 82: 6-13.

Stage G.I. 1966: Biology and Systematics of the American Species of the Genus Hesperapis Cockerell. University of Berkley, New York, 464 pp.

Swofford D.L. 2001: PAUP 4.0b10. Sinauer Associates, Sunderland, $144 \mathrm{pp}$.

Whiте F. 1986: Vegetation of Africa - A Descriptive Memoire to Accompany the UNESCO/AETFAT/UNSO Vegetation Map of Africa. Natural Resources Research Report (20). UNESCO, Paris, 384 pp.

WhiteheAd V.B. \& Steiner K.E. 2001: Oil-collecting bees of the winter rainfall area of South Africa (Melittidae, Rediviva). Ann. Sth Afr. Mus. 108(2): 143-277.

Received June 28, 2006; revised and accepted January 31, 2007 